\title{
UNIVERSITÄT
}

LEIPZIG

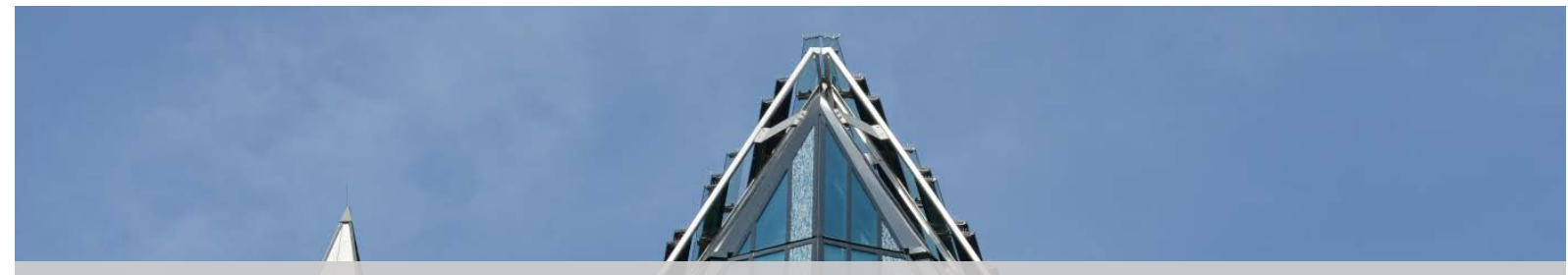

\section{Empirischer Vergleich von KBV und ÖPP}

Studie zu Beschaffungsmethoden der öffentlichen Hand vor dem Hintergrund des Gemeinsamen Erfahrungsberichts der Rechnungshöfe

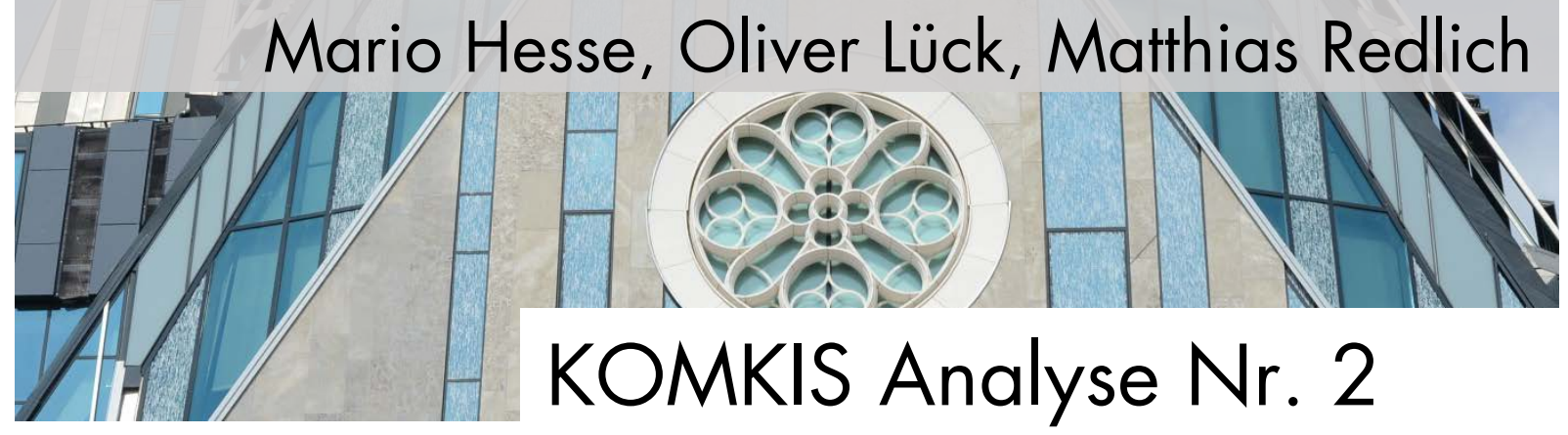


KOMKIS Analyse | Empirischer Vergleich von KBV und ÖPP

Bibliographische Information der Deutschen Nationalbibliothek: Die Deutsche Nationalbibliothek verzeichnet diese Publikation in der Deutschen Nationalbibliographie; detaillierte bibliographische Daten sind im Internet über http:// dnb. d-nb. de abrufbar.

\section{ISSN 2511-7432}

Erscheinungsjahr 2013

\section{Ansprechperson: Mario Hesse}

T +49341 9733-624 | F + 49341 9733-589 | hesse@wifa.uni-leipzig.de

(c) Kompetenzzentrum für kommunale Infrastruktur Sachsen; alle Rechte vorbehalten. Städtisches Kaufhaus, Universitätsstraße 16, 04109 Leipzig.

Foto: Paul Trainer | Universität Leipzig 


\section{Zusammenfassung der Studie}

Seit einigen Jahren erfolgt in Deutschland eine verstärkte Einbindung von privatwirtschaftlichen Akteuren bei der Bereitstellung öffentlicher Leistungen. Insbesondere im Bereich der Infrastrukturbereitstellung haben sich Öffentlich-Private-Partnerschaften als eine alternative Beschaffungsvariante etabliert. Diese Vertrags-ÖPP sind in den Kontext einer allgemeinen Privatisierungskritik geraten, vor allem wird die Wirtschaftlichkeit dieser Variante bzw. deren Berechnung diskutiert. Vor diesem Hintergrund liegt das Ziel der Studie darin, die geringe empirische Datenlage zum Status quo der Umsetzungspraxis zu vergrößern, die Beschaffungsalternativen konventionelle Beschaffungsvariante (KBV) und ÖPP komparativ gegenüberzustellen und dabei auch auf Aspekte einzugehen, die im Erfahrungsbericht der Rechnungshöfe nicht behandelt werden, da dieser ausschließlich die Wirtschaftlichkeit von ÖPP fokussiert. Der Untersuchungsgegenstand fokussiert nur kommunale ÖPP-Projekte die einen Lebenszyklusansatz verfolgen, folglich Infrastruktureinrichtungen wie Schulen, Kindertagestätten und Verwaltungsgebäude. Allen diesen Projekten ist inhärent, dass während der Vertragslaufzeit kein öffentliches Eigentum veräußert wird, die Aufgabenverantwortung folglich stets bei der öffentlichen Hand verbleibt und somit auch keine Privatisierung öffentlichen Eigentums erfolgt. Die Studie zeigt, dass bei vielen KBV-Projekten die Betriebskosten in den anfänglich zu erstellenden Wirtschaftlichkeitsvergleichen keine oder nur eine untergeordnete Rolle spielen, während bei ÖPP in der Regel von Anfang an der gesamte Lebenszyklus der Infrastruktureinrichtung betrachtet wird, da der Großteil der Gesamtkosten der Infrastrukturmaßnahme während der Betriebsphase anfällt. (Kostensparende) Synergieeffekte bleiben deshalb bei den KBV vielfach ungenutzt. Die Nutzung von $A B C$-Ausschreibungen löst dieses Problem nur bedingt, da durch sie Aufwand und Transaktionskosten enorm gesteigert werden.
Die Betrachtung unterschiedlicher Wertschöpfungsstufen (Planung, Bau, Finanzierung, Betrieb, Verwertung) stellt den entscheidenden Punkt für die Wirtschaftlichkeit von ÖPP-Projekten im Vergleich zur KBV dar, da die private Seite veranlasst ist, die Bauleistungen betriebsoptimierend $\mathrm{zu}$ planen und durchzuführen. Ferner zeigt die Studie, dass die von den Rechnungshöfen angemahnte Verfahrenstransparenz eine Herausforderung bei allen öffentlichen Beschaffungsvarianten darstellt und das Fehlanreize, die durch die Prinzipal-Agent-Problematik entstehen, ebenfalls kein singuläres Defizit des ÖPP-Ansatzes sind. Dem Vorwurf, dass ÖPP-Projekte dazu genutzt würden, Schulden in öffentlichen Haushalten zu verschleiern und Kosten möglichst intransparent darzustellen, wird mit der schrittweisen bundesweiten Umstellung der Kommunen auf doppische Haushaltsführung weitestgehend der Boden entzogen, da ÖPP-Projekte mit Forfaitierung sogar Transparenzvorteile gegenüber den KBV aufweisen. Ziel der Studie ist nicht, die ÖPP als „Königsweg" der öffentlichen Beschaffung darzustellen, sondern den Versuch zu unternehmen, einen kritischen Blick auf die Beschaffungsvariante selbst und die an ihr geäußerte Kritik zu werfen. Es gilt: Vor dem Hintergrund der langfristigen Bindung des privaten Partners und der damit verbundenen Verantwortungs- und Risikoteilung ist es nach wie vor möglich, dass wirtschaftlichere und bedarfsgerechtere Lösungen als bei der KBV gefunden werden. 


\section{Inhaltsübersicht}

I Einleitung

II Der "Gemeinsame Erfahrungsbericht" der Rechnungshöfe und

kritische Positionen zu ÖPP

III Design der Studie und der empirischen Erhebung

IV Allgemeiner statistischer Überblick über die befragten Kommunen 17

$\begin{array}{lll}V & \text { Rechtlicher Rahmen und Privatisierung öffentlichen Eigentums } & 20\end{array}$

VI Die Feststellung der Wirtschaftlichkeit bei KBV und ÖPP 22

$1 \quad$ Gesetzliche Regelungen zur Wirtschaftlichkeitsuntersuchung 22

2 Ermittlung des PSC $\quad 25$

$3 \quad$ Wirtschaftlichkeitsvergleich 28

$4 \quad$ Parallelausschreibung 34

$5 \quad$ Vergabekriterien (Lebenszyklusansatz) 37

$6 \quad$ Einschätzung der Projektrisiken $\quad 40$

VII Transparenz und bürgerschaftliche Beteiligung 42

$1 \quad$ Einbeziehung und Information der Öffentlichkeit 43

2 Projektsteverung 46

3 Haushaltstechnische Behandlung 51

VIII Abschließender Vergleich zwischen konventioneller und ÖPP-Realisierung 54 


\section{Abbildungsverzeichnis}

Abb. 1: Durchführungsverantwortung bei ÖPP und KBV im Vergleich.

Abb. 2: $\quad$ Geografische Verteilung der teilnehmenden Kommunen.

Abb. 3: $\quad$ Ausmaß des Sanierungs- und Instandhaltungsstaus.

Abb. 4: Instandhaltungs- und Sanierungsstau pro Kopf.

Abb. 5: $\quad$ Führen Sie bei der KBV im Normalfall eine WU durch?

Abb. 6: Bedurfte das ÖPP einer Genehmigung der Rechtsaufsicht?

Abb. 7: Welche externen Beraterleistungen haben Sie in Anspruch genommen?

Wie hoch beliefen sich jeweils die angefallenen Kosten in Relation zum gesamten Projektvolumen?

Abb. 8: $\quad$ Wie erfolgte die Festlegung der Annahmen für den PSC in den folgenden Bereichen?

Abb. 9: Genutzte Instrumente des Wirtschaftlichkeitsvergleichs der KBV.

Abb. 10: Anteil an der Erstellung der Wirtschaftlichkeitsuntersuchung.

Abb. 11: Vergleich der errechneten Effizienzrendite von Beratern/Verwaltungen und Rechnungshöfen.

Abb. 12: Nutzung von ABC-Ausschreibungen insgesamt. 36

Abb. 13: Kriterien der Vergabeentscheidung bei der KBV. 38

Abb. 14: Kriterien der Vergabeentscheidung bei ÖPP-Projekten. 39

Abb. 15: Durchschnittliche Gewichtung der Vergabekriterien bei der KBV. 40

Abb. 16: Durchführung von Risikoanalysen im Rahmen der KBV. 41

Abb. 17: Berücksichtigung von Risikoanalysen beim Vergleich von KBV und ÖPP? 42

Abb. 18: Information der Bürger bei ÖPP-Projekten. 44

Abb. 19: Information der Bürger bei konventionellen Projekten. 45

Abb. 20: Anwendung von Governance- und Steverungsmechanismen bei konventionellen Projekten. $\quad 47$

Abb. 21: Anwendung von Governance- und Steuerungsmechanismen bei ÖPP-Projekten. 49

Abb. 22: Aufgaben der Projektgruppe bei konventionellen Projekten. 50

Abb. 23: Zeitpunkt der Einführung der Doppik in den Kommunen. 53

Abb. 24: Decken sich die ursprünglichen Annahmen der WU mit den tatsächlichen Ergebnissen? 


\section{Einleitung}

Vor dem Hintergrund der sich zuspitzenden demografischen Entwicklung und einer persistent angespannten Haushaltssituation aller Staatsebenen stehen insbesondere die Kommunen großen finanziellen Herausforderungen gegenüber. Städte, Gemeinden und Landkreise' erbringen neben der gesetzlich vorgeschriebenen Bereitstellung technischer und sozialer Infrastruktur (z. B. Wasserversorgung, Abfallentsorgung, Verkehrswege, Schulen, Kitas, Sozialeinrichtungen) in ihrem Kompetenzbereich ebenfalls freiwillige Aufgaben (z. B. in den Bereichen Sportstätten, Kultur oder Wirtschaftsförderung) für ihre Bürger. Zudem werden die Kommunen von den höheren Verwaltungsebenen mit der Erstellung von Leistungen beauftragt, bei denen die Entscheidungsverantwortung nicht bei ihnen liegt (v. a. soziale Leistungen). Bei diesem Spagat zwischen Anforderungen, Erwartungen und Möglichkeiten ist das Verwaltungshandeln seit Ende des vergangenen Jahrhunderts immer mehr in die Kritik geraten. Bürgerorientierung und Wirtschaftlichkeit der Leistungserbringung wurden dabei die Kernfelder von Reformen. Die Forderung nach der Übertragung von ökonomischen Effizienzpostulaten auf das Verwaltungshandeln veranlasste die öffentliche Hand, neue Wege zu beschreiten, um die ihr zur Verfügung stehenden knappen Ressourcen optimal einzusetzen. In diesem Zusammenhang erfolgt seit einigen Jahren eine verstärkte Einbindung von privatwirtschaftlichen Akteuren bzw. eine Neujustierung der Aufgabenverteilung zwischen öffentlicher Hand und Privatwirtschaft bei der Bereitstellung öffentlicher Leistungen. Neben den aus unterschiedlichen Beweggründen - teilweise gerechtfertigt und teilweise unreflektiert, ideologisch motiviert geäußerten Bedenken und kritisierten Entwicklungen bei der Veräußerung öffentlichen Eigentums (materielle bzw. "echte" Privatisierung), kam es vor allem zu formellen Privatisierungen (Rechtsformänderungen) und organisatorischen
Umstrukturierungen der öffentlichen Aufgabenwahrnehmung. In den letzten Jahren ist der Fokus hingegen verstärkt auf einen neuen Mittelweg zwischen (materieller) Privatisierung und verwaltungsinterner Wahrnehmung gerückt: Öffentlich-Private Partnerschaften (ÖPP) haben sich insbesondere im Bereich der Infrastrukturbereitstellung als eine alternative Beschaffungsvariante für die öffentliche Hand etabliert.

Bei einer ÖPP wird grundsätzlich ein langfristiger Kontrakt zwischen der öffentlichen Hand und einem privaten Partner geschlossen (üblich sind 15-30 Jahre), der in der Regel alle Wertschöpfungsstufen einer Infrastruktureinrichtung (Planung, Bau, Betrieb, Finanzierung, Verwertung) umfasst. Diese Form der Infrastrukturbeschaffung ermöglicht es, Effizienzpotentiale zu nutzen, die erst durch die lebenszyklusorientierte Betrachtung aufgedeckt werden können und die bei der konventionellen Beschaffungsvariante (KBV) derzeit nicht bzw. nur unzureichend genutzł werden. Die langfristige vertragliche Bindung und das frühzeitige Einbinden eines privaten Partners erlauben es auf der einen Seite, das Wissen und die Innovationsdynamik der privaten Wirtschaft sowie wirtschaftliche Anreize bereits bei der Planung in den öffentlichen Beschaffungsprozess einzubinden und die plangemäße Erfüllung über den gesamten Lebenszyklus abzusichern. Die partnerschaftliche Rolle der öffentlichen Hand sichert andererseits, dass deren Leistungsansprüche adäquat durchgesetztund ihre Einflussmöglichkeiten insbesondere durch den Erhalt der Eigentumsverhältnisse auch zukünftig sichergestellt werden. Im Gegensatz zu anderen Beschaffungsvarianten entsteht dabei eine über die typischen AuftraggeberAuftragnehmer-Beziehungen hinausreichende Verbindung, die eine vertrauensvolle und 
erfolgreiche Zusammenarbeit begründet bzw. voraussetzt. Allerdings sind mit der ÖPP-Beschaffungsmethode ebenso spezifische Kosten und Risiken verbunden, die adäquat berücksichtigt werden müssen. Aus diesem Grund ist eine vergleichende Wirtschaftlichkeitsuntersuchung zur Abwägung der existierenden finanziellen Vor- und Nachteile unabdingbar.

Seit dem Jahr 2002 wurden in Deutschland 7,3 Mrd. Euro in ÖPP-Projekte investiert, wobei der Großteil davon auf Projekte im Hochbau (4,9 Mrd. Euro) entfällt. ${ }^{2}$ Seit dem Jahr 2007 ist die jährliche Anzahl der Projekte allerdings zurückgegangen. Auch das jährliche Umsatzvolumen erreichte 2007 seinen Höhepunkt (1,5 Mrd. Euro) und ist seitdem absteigend. Im Jahr 2012 sind insgesamt acht Projekte mit einem finanziellen Volumen von 150 Mio. Euro umgesetzł worden. ${ }^{3}$

Die derzeitig nicht einfache Situation für die ÖPP-Beschaffung wird von Branchenvertretern vielfach auf die gesamtwirtschaftlichen Rahmenbedingungen, insbesondere die Auswirkungen der Finanz- und Wirtschaftskrise, sowie die Effekte des Konjunkturpaktes II zurückgeführt. ${ }^{4}$ Doch darf nicht verkannt werden, dass ÖPP in der öffentlichen Diskussion z. T. kritisch gesehen wird. Vor allem die WirtschaftlichkeitderÖPP-Beschaffungsvariante bzw. deren Berechnung wird dabei zunehmend diskutiert. Bei einigen Projekten haben zu hohe Erwartungen, aber auch teilweise auftretende Probleme und interessengeleitete Betrachtungen die Zweifel von Kritikern beflügelt. OPP ist vor diesem Hintergrund in den Kontext einer allgemeinen Privatisierungskritik geraten, die aufgrund einer prinzipiellen Ablehnung eine sachlich geführte, öffentliche Diskussion und Auseinandersetzung über
Defizite, Anwendungs- oder Methodenfehler, aber auch über vorhandene Vorteile und den Nutzen dieser Beschaffungsvariante erschwert. Vielleicht zur rechten Zeit erschien deshalb Ende des Jahres 2011 der "Gemeinsame Erfahrungsbericht zur Wirtschaftlichkeit von ÖPP-Projekten"5 der Präsidentinnen und Präsidenten der Rechnungshöfe des Bundes und der Länder. In diesem wird gleich zu Beginn unterstrichen, dass ÖPP "eine wertneutrale Beschaffungsvariante zu konventionellen Bau- und Finanzierungsmodellen darstellt" ${ }^{\prime}{ }^{6}$ Trotz dieser Einschätzung stellt der Bericht „nüchtern" die bei der OPP-Realisierung in einigen Projekten aufgetretenen Umsetzungsund Verfahrensschwierigkeiten heraus. Dies erfolgt anhand der detaillierten Untersuchung von einzelnen ÖPP-Projekten. Die Analysen beschränken sich jedoch vielfach auf Teilaspekte. Die gesehenen Kritikpunkte werden aus konkreten Einzelfällen gewonnen, doch wird dabei unzureichend differenziert, ob es sich um bei Anwendung und Umsetzung aufgetretene, projektspezifische oder dem Verfahren immanente Defizite handelt. Ebenso können neue Entwicklungen, Änderungen und Anpassungen der Durchführungspraxis - als Reaktion auf geäußerte Defizite von den Rechnungshöfen nur verzögert abgebildet werden. Darüber hinaus lässt der Gemeinsame Erfahrungsbericht offen, inwiefern die gefundenen Probleme schwächer, ebenso oder sogar noch stärker bei der konventionellen Beschaffung auftreten. Eine vergleichende Gegenüberstellung beider Beschaffungsvarianten in Hinblick auf die Prüferfahrung findet nicht statt.

Ungeachtet dieser kritischen Anmerkungen sind mit den Analysen des Gemeinsamen Erfahrungsberichts der Rechnungshöfe

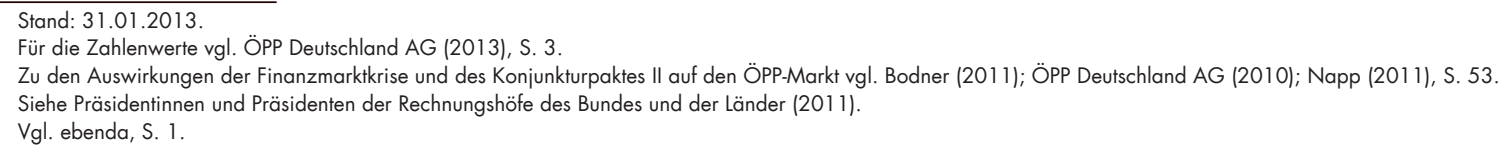


fundierte Ergebnisse zu Problemen bei Wirtschaf tlichkeitsuntersuchungen vorgelegt worden. Um ÖPP und konventionelle Beschaffungsvariante (KBV) objektiv zu vergleichen, gilt es nun, die von den Rechnungshöfen geäußerten Kritikpunkte an der Durchführungspraxis beider Beschaffungsvarianten zu spiegeln.

An diesem Punkt setzł die vorliegende Studie an. Das Ziel besteht darin, die zum Teil ideologiegefärbte Debatte mit empirischem Datenmaterial zu unterfüttern und die geringe empirische Datenlage zum Status quo der Umsetzungspraxis der Beschaffungsalternativen KBV und ÖPP zu vergrößern, ${ }^{7}$ um dabei die weiteren Forschungsbedarfe zu verdeutlichen. Die geäuBerten Kritikpunkte an ÖPP - insbesondere der Rechnungshöfe - werden dazu an der Praxis der KBV reflektiert. Auf diese Weise kann zumindest aus dieser Perspektive ein Vergleich der beiden Beschaffungsvarianten gezogen werden.

Zunächst werden im Kapitel II unter besonderer Berücksichtigung der Erfahrungen der Rechnungshöfe die Kernpunkte der Diskussion um ÖPPerörtert. Bevor diese detailliertbetrachtet werden, wird in Kapitel III das Studiendesign vorgestellt und in Kapitel IV ein statistischer Überblick über die teilnehmenden Kommunen gegeben. Die Auseinandersetzung um den rechtlichen Rahmen und die Privatisierung öffentlichen Eigentums wird in Kapitel V knapp dargestellt. Einen Schwerpunkt der Betrachtung bildet Kapitel VI. Dieses setzt sich mit der Diskussion um den ökonomischen Rahmen des ÖPP-Verfahrens auseinander. Dabei werden die beiden Beschaffungsalternativen KBV und ÖPP anhand der im Gemeinsamen Erfahrungsbericht der Rechnungshöfe dargestellten Hauptkritikpunkte komparativ gegenübergestellt. Anschließend folgt in
Kapitel VII die vergleichende Betrachtung von Transparenz und bürgerschaftlicher Beteiligung bei KBV und ÖPP. Im abschließenden Kapitel VIII werden die gefundenen Ergebnisse zusammengefasst und an den vorhandenen Praxiserfahrungen mit ÖPP gespiegelt. Dabei wird ein vorläufiges Fazit gezogen und auf weitere Forschungsbedarfe verwiesen.

Unter konventioneller Beschaffung wird im Rahmen dieser Studie das Verfahren verstanden, das in der Praxis der Länder und Kommunen üblich ist. Die Planung der Infrastruktureinrichtung erfolgt durch die öffentliche Hand oder mit deren Vorgaben durch private Architekturbüros. Die Bauleistungen werden separat per Ausschreibung nach Teilleistungen/Losen an privatwirtschaftliche Unternehmen vergeben. Der Betrieb wird durch die öffentliche Hand (eigenes Personal und eigene Sachmittel) oder - partiell oder vollständig - durch einen/mehrere Private(n) erfüllt. Finanziertwerden die Einrichtungen aus Eigenmitteln oder ggf. aus kreditfinanzierten Einnahmen, die Betriebsleistungen werden aus der laufenden Rechnung bestritten. Die Verwertung der Einrichtungen am Ende ihrer Nutzungsdauer wird durch den offentlichen Eigentümer gestevert. An allen Schnittstellen zwischen den Schritten des Lebenszyklus sowie insbesondere zwischen den Gewerken bei Bau und Betrieb der Einrichtungen liegen die Aufgaben der Koordination und Steuerung bei der öffentlichen Hand. Dies gilt auch für die Generalunternehmervergabe, bei der die Bauleistungen bei einem privaten Unternehmen als Vertragspartner der öffentlichen Hand gebündelt werden und insofern die Organisation der Schnittstellen an diesen übergeht. 
Charakteristisch für Öffentlich-Private Partnerschaften ist demgegenüber die Leistungserbringung durch einen für mehrere Stufen des Lebenszyklus' durchführungsverantwortlichen ${ }^{8}$ privaten Partner, während die öffentliche Hand in allen Phasen die Aufgabenverantwortung ${ }^{9}$ trägt und damit die wesentlichen Zielvorgaben bestimmt. ${ }^{10}$ In Abbildung 1 sind beispielhaft idealtypische Varianten der Verteilung der Durchführungsverantwortung in den einzelnen Wertschöpfungsstufen bei ÖPP und KBV dargestellt. Die Farben repräsentieren die unterschiedlichen Akteure. Anders als bei der KBV können dadurch Planung, Bau und Betrieb und ggf. auch Finanzierung und Endverwertung integriert betrachtet und realisiert werden. Effizienzvorteile können sich dabei einerseits aus einer umfangreicheren, den Betrieb berücksichtigenden Planung ergeben, die eine höhere Effizienz in der Betriebsphase ermöglicht. Andererseits werden u. a. durch die Übertragung der Durchführungsverantwortung mehrerer Phasen auf einen privaten Partner und die Risikoteilung wettbewerbliche Anreizstrukturen geschaffen, die zugleich die für öffentliche Auftraggeber bestehende Schnittstellenproblematik entschärft und damit Vorteile bietet. Klar zuweisbare Verantwortlichkeiten und vertraglich festgelegte Leistungen, Qualitäten, Fristen und Preise können zudem positive Effekte insbesondere in der Erstellungs-/Bauphase ermöglichen.

\section{Abbildung 1: Durchführungsverantwortung bei ÖPP und KBV im Vergleich.}

Status quo der öffentlichen Beschaffung (Vergabe in Fachlosen)

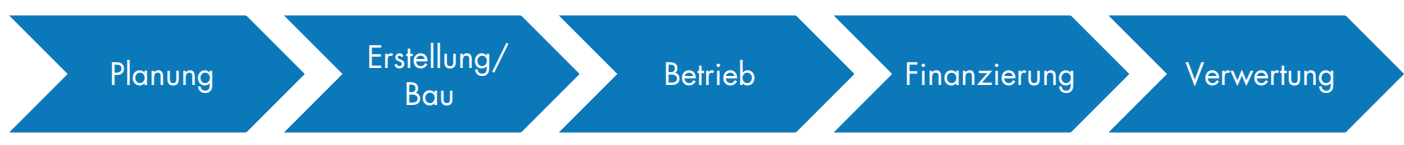

Status quo der öffentlichen Beschaffung (GU-Vergabe)

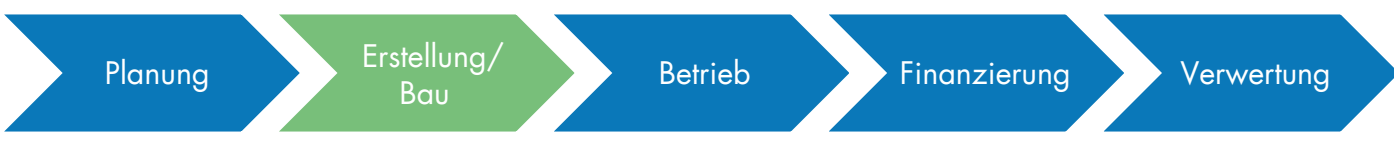

Alternativen

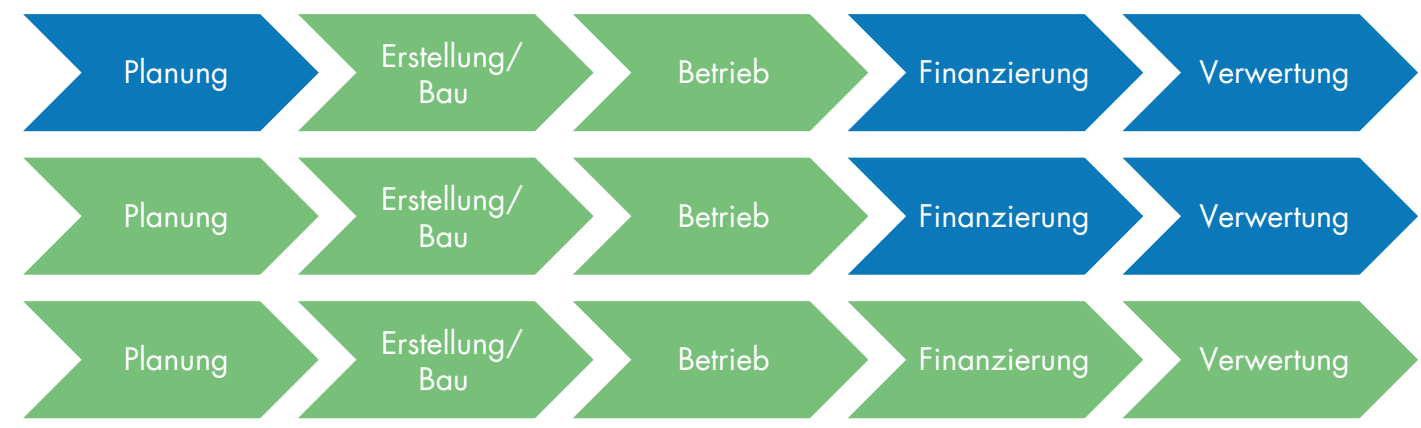

blau = bei öffentlicher Hand, grün = beim privaten Unternehmen

Quelle: Eigene Darstellung.

$8 \quad$ Unter Durchführungsverantwortung wird die Verpflichtung der übergreifenden Koordination und Steuerung der Leistungserbringung in einer Phase verstanden.

Die Leistungserbringung kann dabei aber von verschiedenen Akteuren erbracht werden.

Aufgabenverantwortung wird verstanden als Pflicht, Leistungen im öffentlichen Interesse zu erbringen und dafür im Rahmen von Beschaffungsmaßnahmen wesentliche Zielvorgaben für

Projekte zu formulieren und durchzusetzen.

10 Siehe dazu in dieser Studie Kapitel V Rechtlicher Rahmen und Privatisierung öffentlichen Eigentums. 


\section{Der "Gemeinsame Erfahrungsbericht" der Rechnungshöfe und kritische Positionen zu ÖPP}

Zahlreiche Informationsveranstaltungen, Projektpräsentationen und Studien weisen immer wieder auf die vielfältigen Vorteile von ÖPP hin: Zufriedenheit der Auftraggeber mit Bauzeit, Qualität und Preis während der Errichtung und im Betrieb; Zufriedenheit der Nutzer mit Raumgestaltung, Einflussmöglichkeiten, Schnelligkeit und Ausführung von Wartungs-, Instandsetzungs- und Betriebsleistungen sowie der Erreichbarkeit von Ansprechpartnern. ${ }^{11}$ Hinzu kommt die Zufriedenheit der Verwaltung mit Betriebsaufwendungen, -leistungen und -kosten sowie dem eigenen personellen Einsatz. Während viele an der Umsetzung von ÖPP-Projekten beteiligte Akteure dieses positive Bild zeichnen, gibt es aber auch Stimmen, die diese Beschaffungsform kritisch sehen und auf die aus ihrer Sicht bestehenden Defizite aufmerksam machen. ${ }^{12}$ In der Diskussion bildeten sich dahingehend drei Schwerpunktbereiche heraus:

Rechtlicher Rahmen und Privatisierung öffentlichen Eigentums,

Ökonomischer Rahmen und Wirtschaftlichkeitsvergleich sowie

Transparenz und bürgerschaftliche Beteiligung.

Der rechtliche Rahmen und die institutionellen Beschaffungsmöglichkeiten der Kommunen sowie privatisierungs- und steuerungskritische Debatten sind seit spätestens Ende der 1990er Jahre fester Bestandteil der wissenschaftlichen (und auch öffentlichen) Auseinandersetzung. ${ }^{13}$ Im Erfahrungsbericht der Rechnungshöfe wird - da dieser die Wirtschaftlichkeit von ÖPP fokussiert - auf diese übergreifenden Problematiken nicht explizit eingegangen. Dessen ungeachtet wird OPP immer wieder das Etikett der
"Privatisierung des öffentlichen Eigentums" angeheftet. Insbesondere Kritiker versuchen damit, die zum Teil vorherrschenden Ressentiments in der Bevölkerung gegenüber einen "Ausverkauf des öffentlichen Eigentums" zu bedienen. ${ }^{14}$ Im Zuge der privatisierungskritischen Diskussion wurde immer wieder das aus der Prinzipal-Agenten Theorie bekannte Problem der Informationsasymmetrie, aber auch die Problematik langfristiger Verträge, (un-)demokratischer Entscheidungsstrukturen und staatlicher Aufgabenverantwortung eingebracht. Daraus hat sich in jüngster Zeit ein Themenbereich entwickelt, der um Transparenz - des Entscheidungsfindungsprozesses auf der einen und der geschlossenen Verträge auf der anderen Seite - sowie um die bürgerschaftlichen Beteiligungs- und Mitbestimmungsmöglichkeiten am Entscheidungsprozess kreist. ${ }^{15}$ Den Kernbereich der Auseinandersetzung um ÖPP bildet jedoch der ökonomische Rahmen. Die öffentliche und fachliche Diskussion dreht sich dabei immer wieder um den Wirtschaftlichkeitsvergleich von ÖPP und KBV. ${ }^{16}$ Die diesbezüglich existierenden Kritikpunkte werden im Gemeinsamen Erfahrungsbericht der Rechnungshöfe zur Wirtschaftlichkeit von ÖPPProjekten aufgegriffen.

Wie bereits erwähnt, wird ÖPP von den Rechnungshöfen als eine "wertneutrale Beschaffungsalternative" zur KBV angesehen, deren Vorteilhaftigkeit durch einen objektiven und transparenten Wirtschaftlichkeitsvergleich belegt werden muss. ${ }^{17}$ Die Rechnungshöfe führen zwei wesentliche Grundsätze an, die für die Realisierung jedes ÖPP-Projekts unabdingbare Voraussetzungen darstellen: Zum einen sollte die öffentliche Hand nur dann ein Infrastrukturprojekt als ÖPP durchführen, wenn sie es sich auch auf konventionellem

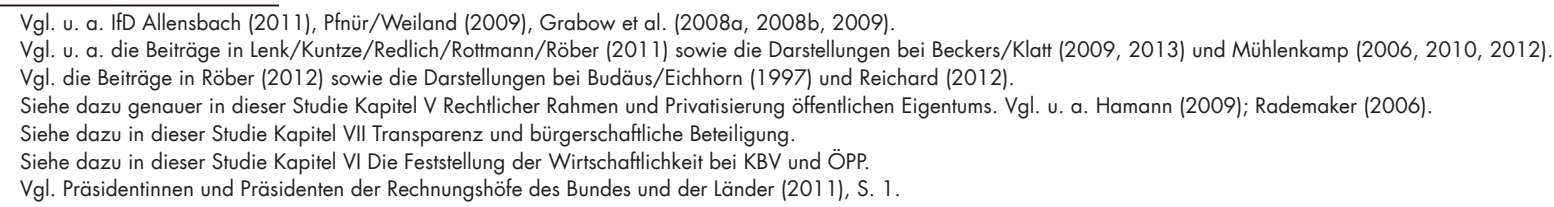


Wege leisten kann. Zum anderen muss das ÖPP-Projekt über die gesamte Vertragslaufzeit nachweislich wirtschaftlicher sein als eine Realisierung über die KBV.

Der Gemeinsame Erfahrungsbericht macht dabei auf verschiedene Schwierigkeiten aufmerksam, die bei der Prüfung von sehr unterschiedlichen ÖPP-Projekten aufgedeckt wurden. Diese betreffen hauptsächlich die Bereiche

[ Finanzierung von ÖPP-Projekten,

- Aspekte des Lebenszyklusansatzes,

die Ermittlung des Public Sector Comparators (PSC) sowie

die Risikobewertung und die Risikoverteilung.

Ziel des Berichts der Rechnungshöfe ist es, die öffentliche Hand für Probleme bei der Anwendung des ÖPP-Verfahrens zu sensibilisieren. Einzelfallbezogene Umsetzungsdefizite werden von ihnen dabei angemahnt. Nichtsdestotrotz sind die an die alternative Beschaffungsvariante gestellten Ansprüche mit der Realität der konventionellen Beschaffungspraxis zu vergleichen und ebenso vor diesem Hintergrund zu bewerten. Nur diese Vorgehensweise verdeutlicht,

welche Defizite erst durch das ÖPP. Verfahren entstehen oder durch dieses virulent werden,

- welche Defizite grundsätzlich ebenfalls oder sogar in noch größerem Ausmaß auch bei der KBV auftreten und

- welche Defizite durch die Nutzung dieser alternativen Beschaffungsalternative gelöst werden können.
In der öffentlich-medialen Diskussion über ÖPP, die von einer gewissen Grundskepsis geprägt ist, wird diese notwendige Abgrenzung vielfach nicht vorgenommen. Auch im Gemeinsamen Erfahrungsbericht wird keine vergleichende Betrachtung durchgeführt. Vielmehr konzentriert sich dieser lediglich auf die Wirtschaftlichkeit von ÖPP-Projekten. In dieser Perspektive erscheinen jedoch alle Defizite allein dem ÖPP-Ansatz geschuldet zu sein. Überdies mangelt es an empirischen Daten und Studien, die beide Beschaffungsvarianten vergleichend gegenüberstellen und somit die tatsächliche Beschaffungsrealität herausstellen. Diese Studie stellt einen ersten Ansatz dar, dieses Defizit zu lösen. 


\section{Design der Studie und der empirischen Erhebung}

Die vorliegende Studie versucht mit Hilfe von empirischen Daten die Kritikpunkte an ÖPPProjekten mit der Praxis bei konventionellen Projekten zu vergleichen. Die vorliegende Untersuchung kann aufgrund der erheblichen Unterschiede nicht alle Aufgaben- und Anwendungsfelder von ÖPP abdecken. Insgesamt wird der Fokus auf kommunale ÖPP-Projekte gelegt. Die Untersuchung konzentriert sich auf die Neuerrichtung und Sanierung von Infrastruktureinrichtungen in den Bereichen Schule, Kindertagesstätten und Verwaltungsgebäude. In diesen drei Tätigkeitsfeldern liegen im kommunalen Bereich zahlreiche Erfahrungen vor. Über $73 \%$ der realisierten ÖPP-Projekte wurden auf kommunaler Ebene durchgeführt. Dabei finden $61 \%$ der Projekte in diesen Bereichen statt. ${ }^{18}$ Zugleich können hier ebenso für die KBV vielfältige Erfahrungen vorausgesetzt werden. Kindertagesstätten, Schulen und Verwaltungsgebäude existieren in nahezu jeder Kommune. Deren Planung und Realisierung sind (im Gegensatz z. B. zu Schwimmbädern) gut vergleichbar. Zudem haben viele Kommunen aufgrund gesetzlicher Vorgaben oder vorhandener Fördermittel in den vergangenen Jahren mit dem Ausbau und der Sanierung ihrer Einrichtungen begonnen, sodass vielfältige Vergleichserfahrungen vorliegen. Nicht zuletzt bieten sich diese drei Bereiche für die Untersuchung an, da insbesondere bei Kindertageseinrichtungen und Schulen die Nutzereinbindung und die Nutzerzufriedenheit leichter bestimmt und abgefragt werden können.

Um vergleichbare Ergebnisse zu erhalten, wurde eine Teilung der Grundgesamtheit vorgenommen. Anhand der Projektdatenbank der ÖPP Deutschland AG wurden die ÖPPProjekte identifiziert, die in den drei genannten Bereichen in Deutschland bisher durchgeführt wurden. An dieser Stelle ist darauf hinzuweisen, dass in dieser Projektdatenbank nicht alle existierenden ÖPP-Projekte enthalten sind. Insbesondere sind in ihr nur die ÖPPProjekte aufgeführt, die eine lebenszyklusorientierte Betrachtung vorweisen (ÖPP der 2. Generation). ÖPP-Projekte, die lediglich auf den Aspekt der alternativen Finanzierung abstellen (ÖPP der 1. Generation), sind in dieser Auflistung nicht enthalten. Dieser Definition folgend, werden in dieser Arbeit unter dem Begriff der Öffentlich-Privaten Partnerschaft nur jene Projekte subsumiert, die einen Lebenszyklusansatz verfolgen.

In Anbetracht dessen wurden in der ersten Phase die 62 Gemeinden und Landkreise angeschrieben, die laut Projektdatenbank aufgelistet ein oder mehrere Projekte in den Bereichen Schule, Kindertagesstätten und Verwaltungsgebäude vorweisen. Jede Kommune wurde unabhängig von der Anzahl der existierenden Projekte nur einmal befragt. Diesbezüglich fand keine Gewichtung der Antworten statt.

Als Vergleichsgruppe wurde in einer zweiten Phase aus den Kommunen in Deutschland mit einer Größe ab 10.000 Einwohnern eine zufällige Stichprobe von 300 Kommunen gezogen. Dabei wurde darauf geachtet, dass Dopplungen vermieden wurden. Kam es zu dem Fall, dass eine Kommune in beiden Gruppen vertreten war, wurde diese aus der Teilgesamtheit der Vergleichskommunen herausgenommen und durch eine andere, ebenfalls zufällig ausgewählte Kommune ersetzt. Die zufällig ausgewählten Kommunen haben somit nach Maßgabe der ÖPP-Projektdatenbank bisher kein ÖPP-Projekt in einem der drei zu untersuchenden Bereiche durchgeführt.

Der Fragebogen, der an die insgesamt 363 Kommunen und Landkreise verschickt wurde, setzte sich aus mehreren Befragungsteilen zusammen. Im Abschnitt A wurden allgemeine

Eigene Berechnungen auf Grundlage der Angaben der PPP-Projektdatenbank, www.ppp-projektdatenbank.de. 
statistische Daten wie Bevölkerungsgröße oder der Schuldenstand pro Kopf abgefragt, um eine grundsätzliche Einordnung der Kommunen vornehmen zu können. Im zweiten Abschnitt B stand die Beschaffungspraxis der konventionellen Realisierung im Vordergrund. Der Fragebogen der 62 ÖPP-Kommunen enthielt zusätzlich einen dritten Befragungsteil. In diesem Teil C wurden Fragen bezüglich der Erfahrung mit dem bzw. den ÖPP-Projekten gestellt.

Von den 363 verschickten Fragebögen kamen insgesamt 51 ausgefüllte Fragebögen zurück. Die Rücklaufquote liegt damit bei $14 \%$. Auf die beiden Vergleichsgruppen aufgeteilt, beträgt die Rücklaufquote für die ÖPP-Kommunen $11 \%$ (8 Kommunen) und für die Vergleichskommunen $14 \%$ (43 Kommunen). ${ }^{19}$ Die Größe der Stichprobe ermöglicht damit einen groben empirischen Überblick und die Absicherung trendmäßiger Aussagen. ${ }^{20}$ Insbesondere die geringe Anzahl ÖPP-Kommunen kann nur ansatzweise valide Rückschlüsse auf die bereits sehr kleine Grundgesamtheit ziehen und repräsentative Aussagen für ÖPP-Projekte insgesamt treffen. 


\section{Allgemeiner statistischer Überblick über die befragten Kommunen}

Zur besseren Einordnung der antwortenden Kommunen wurden zunächst allgemeine Informationen erfragt. Ein Blick auf die geografische Verteilung der teilnehmenden Kommunen zeigt (Abbildung 2), dass die Mehrzahl von ihnen aus den Bundesländern Nordrhein-Westfalen (21\%), Niedersachsen $(17 \%)$ und Bayern (14\%) stammen. Kommunen aus Thüringen und Rheinland-Pfalz sind nicht vertreten. Auch hat keiner der drei Stadtstaaten teilgenommen. ${ }^{21}$
Aus Nordrhein-Westfalen kamen die meisten Kommunen mit ÖPP-Erfahrung. Knapp ein Drittel der Kommunen, die zusätzlich den ÖPP-Teil der Befragung erhielten, sind aus diesem Bundesland. Keine ÖPP-Projekte aus den drei zu untersuchenden Bereichen gab es hingegen in Berlin, Bremen und dem Saarland.

Von den antwortenden Kommunen konstatierten knapp 65 \% einen spürbaren Instandhaltungsstau bei Schulen, Kindertagesstätten

\section{Abbildung 2: Geografische Verteilung der teilnehmenden Kommunen.}

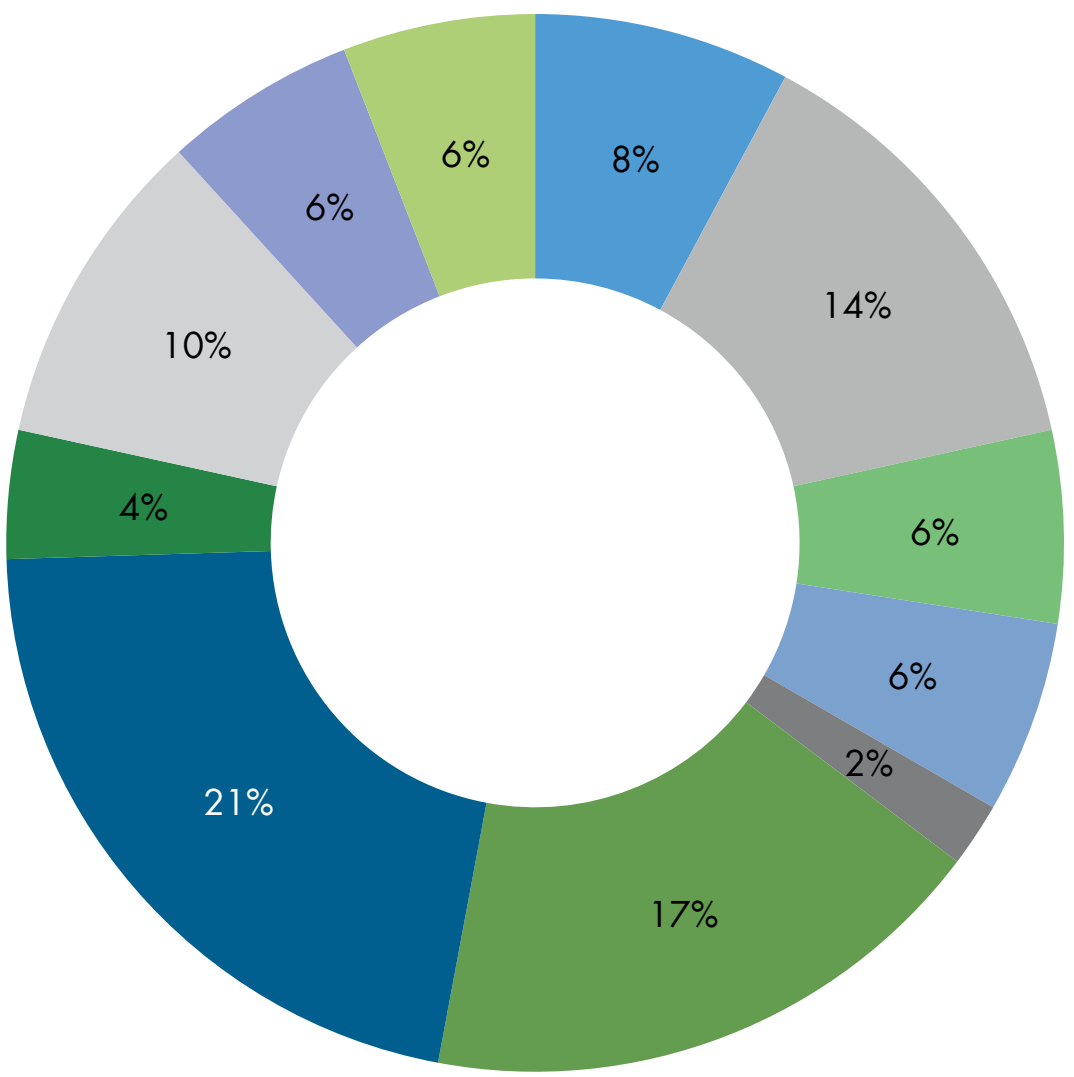

- Baden-Württemberg

Bayern

Brandenburg

- Hessen

$\square \mathrm{MV}$

- Niedersachsen

- NRW

- Saarland

Sachsen

- Sachsen-Anhalt

- Schleswig-Holstein

Quelle: Eigene Erhebung, eigene Darstellung, $\mathrm{n}=51$. 
und/oder Verwaltungsgebäuden. Auf die Frage nach dem Umfang des Instandhaltungsstaus antwortete mehr als die Hälffe der Kommunen (52\%), dass dieser langfristig abbaubar ist. Lediglich $12 \%$ geben an, dass die über die Jahre gemachten Versäumnisse bei der Instandhaltung nicht mehr rückführbar seien. Demgegenüber sahen rund $35 \%$ der Kommunen keinen bzw. nur einen unbedeutenden Rückstand bezüglich der Instandhaltung. ${ }^{22}$

Dieses Ergebnis bestätigł die Vermutung, dass in vielen deutschen Kommunen in den vergangenen Jahren zu wenig finanzielle Mittel für Sanierungs- und Instandhaltung smaßnahmen aufgewendet wurden, um ihre Infrastruktur auf einem adäquaten qualitativen Level zu halten. Nach den genauen Gründen für diese Versäumnisse wurde jedoch nicht gefragt. Es kann aber vermutet werden, dass aufgrund der angespannten Haushaltslage vieler deutscher Kommunen eine oft andere Prioritätensetzung bei den kommunalen Aufgaben erfolgte und demzufolge zumeist keine finanziellen Mittel für Sanierungs- und Instandhaltungsmaßnahmen bereitgestellt werden konnten. So sind gerade die Ausgaben im sozialen Bereich in den vergangenen Jahren stetig gestiegen und zeichnen maßgeblich für die großen finanziellen Schwierigkeiten der deutschen Kommunen mitverantwortlich. ${ }^{23}$ Vor diesem Hintergrund erscheint der Anteil der Kommunen ohne bzw. mit nur geringen Instandhaltungsrückständen überraschend. Die strukturell unausgeglichene Haushaltslage in vielen Kommunen wird in diesem Zusammenhang jedoch durch das kommunale Investitionsprogramm im Rahmen des Konjunkturpakets II überlagert, welches in den Jahren 2009 und 2010 insbesondere im Bildungsbereich die Realisierung notwendiger Instandhaltungsmaßnahmen ermöglicht hatte. ${ }^{24}$ Zudem herrschtoffenbar Optimismus hinsichtlich der Möglichkeiten, Instandhaltungsmaßnahmen langfristig realisieren zu können. Neun von

\section{Abbildung 3: Ausmaß des Sanierungs- und Instandhaltungsstaus.}

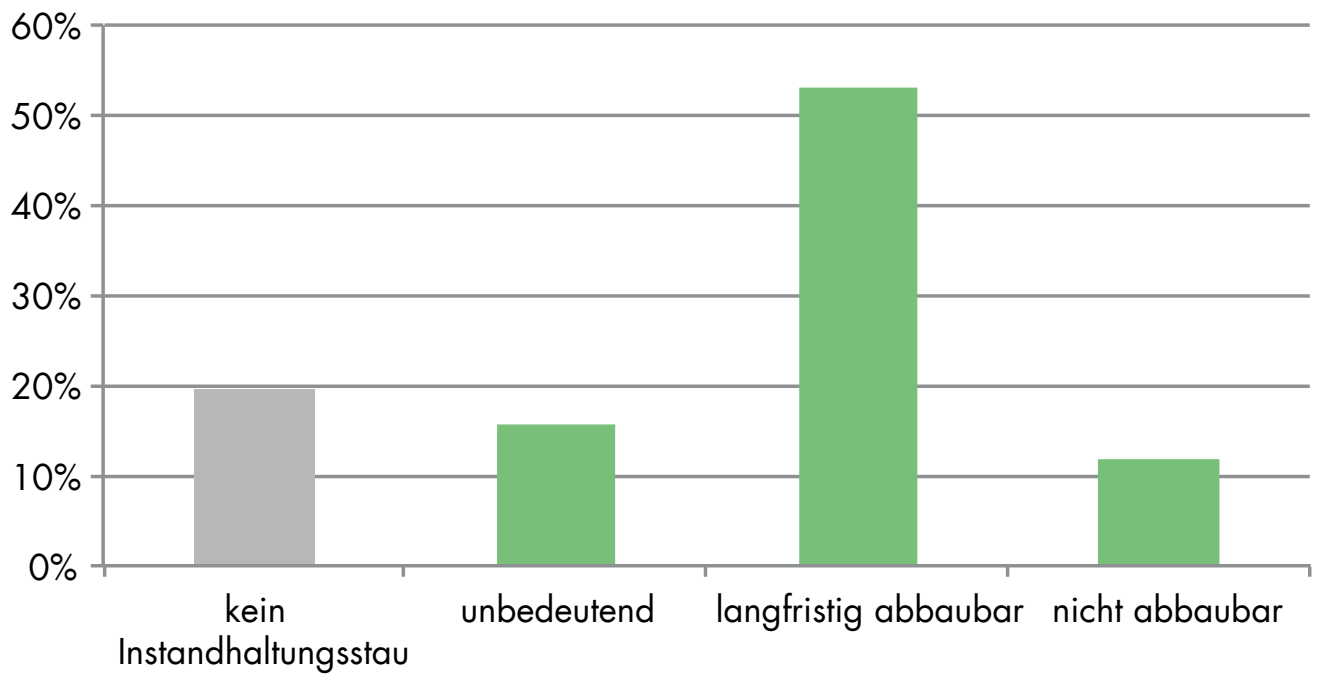

Quelle: Eigene Erhebung, eigene Darstellung, $\mathrm{n}=51$. 
zehn befragten Kommunen mit Rückständen geben dies an. Diese Aussagen mögen überraschend wirken, kontrastieren sie doch den regelmäßig publizierten Investitionsrückstand der Kommunen. ${ }^{25}$ Diese Diskrepanz erklärt sich durch die unterschiedliche Abgrenzung der Bereiche. Während Investitionen der Kapitalrechnung zuzurechnen sind (Vermögenshaushalt der Kameralisitik), zählen Instandhaltungsaufwendungen zur laufenden Rechnung (Verwaltungshaushalt der Kameralistik). Die Instandhaltung bezieht sich damit auf bereits bestehende Infrastruktureinrichtungen und grenzt sich damit von Neu- und Erweiterungsinvestitionen ab. Auf letztere beziehen sich für gewöhnlich die Umfragen zu Investitionsbedarfen und -rückständen.

Der Bundesdurchschnitt für laufende Instandhaltungsaufwendungen für Grundstücke und baulichen Anlagen im Jahr 2011 betrug rund 48 Euro/Einw. ${ }^{26}$ Bei den Ausgaben für Instandhaltungsmaßnahmen reichte die Spannweite der Antworten der teilnehmenden
Kommunen von 3,08 Euro/Einw. bis zu 219,09 Euro/Einw. Die Mehrzahl der teilnehmenden Kommunen investierte in die Instandhaltung ihrer Infrastruktur einen Betrag im Bereich von 10 bis 40 Euro/Einw.

Es könnte nun vermutet werden, dass die in Abbildung 3 und Abbildung 4 angegebenen Werte in einem direkten Zusammenhang stehen - diejenigen Kommunen, die ein relativ hohes Instandhaltungsvolumen aufweisen, sehen keinen wesentlichen Rückstau, während die Kommunen mit geringen Instandhaltungsbudgets entsprechende Lasten "vor sich her schieben". Dies lässt sich auf Basis der Befragung jedoch nicht bestätigen. Diejenigen Kommunen mit einem unterdurchschnittlichen Ausgabeverhalten für Instandhaltungsmaßnahmen sind genauso optimistisch wie diejenigen mit überdurchschnittlichen Aufwendungen. Auch der Anteil der sich überfordert sehenden Kommunen konzentriert sich nicht im Bereich der geringen Instandhaltungsaufwendungen.

Abbildung 4: Instandhalłungs- und Sanierungsstau pro Kopf.

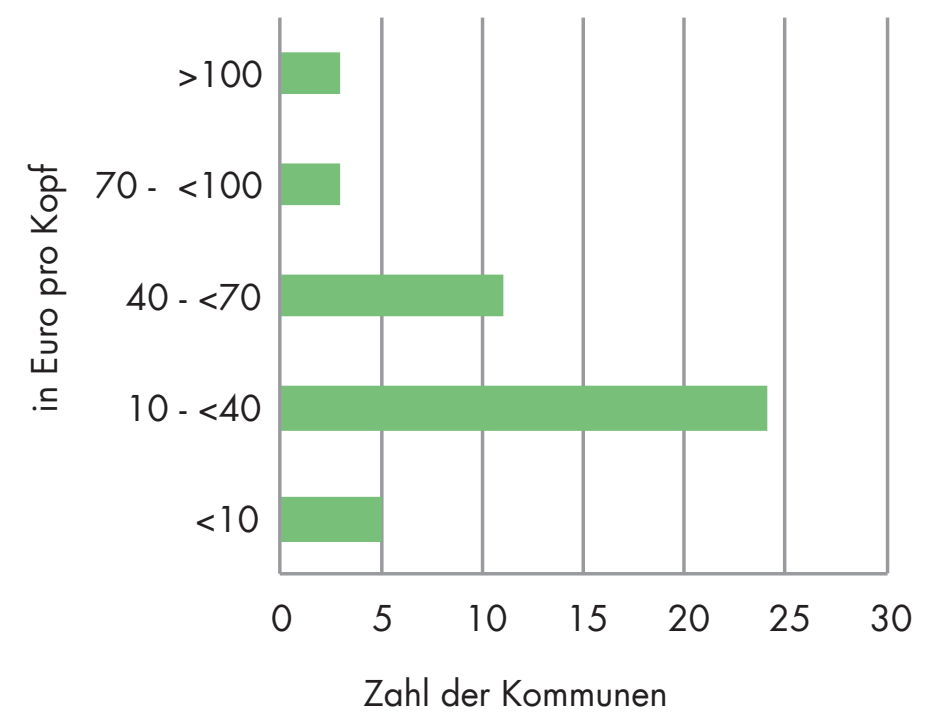

Quelle: Eigene Erhebung, eigene Darstellung, $n=46$. 


\section{Rechtlicher Rahmen und Privatisierung öffentlichen Eigentums}

Die Kritik an der rechtlichen Ausgestaltung von ÖPP und der damit oft verbundene Vorwurf der Privatisierung öffentlichen Eigentums ${ }^{27}$ war nicht zentraler Bestandteil des Erkenntnisinteresses des versendeten Fragebogens. Um die kritische Auseinandersetzung mit ÖPP in ihrer Vielgestaltigkeit abzubilden, wird im Folgenden dennoch kurz auf häufig geäußerte Bedenken eingegangen.

Ein charakteristisches Merkmal von ÖPP ist die langfristige, vertraglich geregelte Zusammenarbeit zwischen der öffentlichen Hand und dem privaten Sektor, die in der Regel meist auf ein bestimmtes Projekt beschränkt ist. Dieses Begriffsverständnis umfasst sowohl die institutionellen als auch die Vertrags-ÖPP. Der Begriff der institutionellen ÖPP rekurriert in erster Linie auf gemischtwirtschaftliche Unternehmen. Sie entstehen durch die Einbeziehung des privaten Partners in die Anteilseignerstruktur eines kommunalen Unternehmens. Insofern gehen sie mit einer Übertragung des öffentlichen Eigentums einher (materielle Teilprivatisierung). In einer institutionellen ÖPP arbeiten die öffentlichen und privaten Partner unbefristet in einem festen institutionellen Arrangement zusammen, in das beide Seiten Ressourcen einbringen (z. B. Stadtwerke mit privatem Anteilseigner). ${ }^{28}$

Während der private Partner über das gemischtwirtschaftliche Unternehmen Leistungen der Daseinsvorsorge zumindest teilweise übernimmt, ist dies bei Vertrags-ÖPP zumeist nicht der Fall. Bei Vertrags-ÖPP kommt es maßgeblich auf die exakte Ausgestaltung der vertraglichen Realisierungsform an. Dabei werden verschiedene (theoretische) ÖPPVertragsmodelle unterschieden. ${ }^{29}$ Das bisher in der Praxis deutschlandweit am häufigsten genutzte Modell für ÖPP-Projekte der zweiten Generation ist das Inhabermodell. Rund $80 \%$ der bisher umgesetzten Vertrags-ÖPP sind in dieser Form ausgestaltet. ${ }^{30}$ Beim Inhabermodell errichtet bzw. saniert der private Partner ein Gebäude aufeinem Grundstück der öffentlichen Hand. Im Falle der Sanierung befindet sich das Gebäude bereits im Eigentum der öffentlichen Hand. Bei dieser Vertragskonstruktion bleibt die öffentliche Hand auch während der gesamten Vertragslaufzeit der wirtschaftliche Eigentümer des Objektes. Die Nutzung des Objektes durch den Auftraggeber während der Vertragslaufzeit ergibt sich laut § 903 BGB automatisch aus seiner Stellung als zivilrechtlicher Eigentümer, die er bereits vor der vollständigen Zahlung der vertraglich festgelegten Entgelte einnimmt. Das Bau, Planungs- und Finanzierungsrisiko liegt im Wesentlichen beim privaten Partner. Zu Beginn der Betriebsphase gehen das allgemeine Sach- und Preisrisiko auf den öffentlichen Auftraggeber über. Somit wird während der gesamten Vertragslaufzeit kein öffentliches Eigentum veräußert. Eine materielle Privatisierung von öffentlichem Vermögen findet nicht statt. $^{31}$ Ebenso bleibt die Aufgabenverantwortung, wie beispielsweise Schulbildung, Kinderbetreuung und das Angebot von Bürgerdiensten inklusive der Formulierung von Zielvorgaben, des Monitorings und der Steuerung des privaten Auftragnehmers bei der öffentlichen Hand.

In der öffentlichen Diskussion werden die beiden unterschiedlichen ÖPP-Formen aber teilweise vermischt. Grundsätzlich werden Vertrags-ÖPP gemeint, wenn von ÖPP gesprochen wird. Eine notwendige differenzierte Betrachtung zu institutionellen ÖPP bzw. eine begriffliche Klarstellung findet in weiten Teilen der öffentlichen Debatte jedoch oft nicht statt. ${ }^{32}$ Die Einbeziehung eines privaten Partners lässt immer wieder die Befürchtung

\footnotetext{
Vgl. u. a. Hamann (2009); Rademaker (2006).

Vgl. Budäus/Grüb (2008), S. 37. Siehe weiter zu institutionellen ÖPPs auch Reichard (2006); Sack (2013).

Siehe zu den ÖPP-Vertragsmodellen genauer Beratergruppe - PPP im öffentlichen Hochbau (2003), S. 90-97.

Vgl. ÖPP Deutschland AG (2013), S. 24.

Vgl. Gatzke (2010), S. 50-53.

Siehe Rösmann (2012), Hamann (2009).
} 
aufkommen, dass dies der erste Schritt zu einer Privatisierung des zivilrechtlichen Eigentums der öffentlichen Hand ist. Der Begriff Privatisierung wird dabei zumeist als Allgemeinbegriff für die Gesamtheit der existierenden Privatisierungsformen verwendet. ${ }^{33}$ Dadurch wird zum Teil eine (negativ konnotierte) Übertragung von Eigentum der öffentlichen Hand suggeriert, die de facto nicht gegeben ist.

Argumentiert wird, dass durch die Übertragung der Durchführungsverantwortung an den privaten Partner vormals von der öffentlichen Hand wahrgenommene Aufgaben insbesondere in der Betriebsphase, nunmehr private Unternehmen einbezogen werden. ${ }^{34}$ Allerdings wird in dieser Betrachtung ausgeblendet, dass bei der KBV die meisten Betriebsaufgaben, wie Wartungs-, Instandhaltungs-, Reinigungs- und diverse Serviceleistungen in der Regel ebenso nicht von öffentlichen Angestellten bzw. öffentlichen Unternehmen erbracht werden, sondern auch hier ein Rückgriff auf private Unternehmen erfolgt. Jedoch tragen in der Betriebsphase bei der KBV vielfach öffentliche Bedienstete Durchführungsverantwortung und sind mit der Koordination und dem Beschwerdemanagement sowie dem Dienstleistungscontrolling und der Auftrags(neu)vergabe von Betriebsleistungen betraut. In der Arbeitspraxis wird diese Verantwortung zumeist von unterschiedlichen Stellen wahrgenommen. Zudem sind mit den einzelnen Leistungen diverse private Unternehmen beauftragt, sodass die Nutzer zumeist keinen einheitlichen Ansprechpartner vorfinden. Insbesondere bei Verwaltungsgebäuden, Schulen und Kindertagesstätten führt dies dazu, dass die Leiter der Einrichtungen oft notgedrungen die ersten Ansprechpartner für Störungen und Probleme jeder Art werden. Obwohl andere Kernaufgaben vorliegen, sind die Leiter somit vielfach "nebenbei" für die Sicherstellung der betrieblichen Serviceleistungen verantwortlich. Die Übertragung der Durchführungsverantwortung auf einen privaten Partner kann in Verbindung mit Service-Level-Agreements, die Anreizstrukturen z. B. in Form von Malus-Regelungen enthalten, und einem aktiven öffentlichen Projekt-Monitoring zu einer verbesserten Leistungserbringung, zur Entlastung der Leitung und zu größerer Nutzerzufriedenheit führen. 


\section{Die Feststellung der Wirtschaftlichkeit bei KBV und ÖPP}

\begin{abstract}
Öffentliche Infrastrukturmaßnahmen binden in der Regel große finanzielle Volumen. Dies betrifft sowohl die Planung und Errichtung never Einrichtungen als auch die Sanierung und Instandsetzung bestehender Infrastrukturbereiche. In Anbetracht der angespannten fiskalischen Situation in den meisten Kommunen und Landkreisen ${ }^{35}$ sind entsprechende Maßnahmen von erheblicher finanzieller Bedeutung. Unabhängig von der gewählten Beschaffungsvariante ist eine wirtschaftliche Realisierung von Planung, Bau und Betrieb deshalb unerlässlich und in Form einer Wirtschaftlichkeitsuntersuchung (WU) nachzuweisen.
\end{abstract}

Die WU ist ein komplexes Verfahren, das mehrere Phasen umspannt. Um einen belastbaren Beleg der Wirtschaftlichkeit einer Beschaffungsvariante abgeben zu können, sind vielfältige Betrachtungen notwendig. Vor diesem Hintergrund gerieten die Methodik der WU in den letzten Jahren insbesondere bei ÖPP-Realisierungen immer häufiger in die Kritik. $^{36}$

In diesem Kapitel wird deshalb untersucht, was die WU von KBV und ÖPP unterscheidet und wie in diesem Zusammenhang die Kritik an der derzeitigen Durchführungspraxis von Wirtschaftlichkeitsvergleichen bei OPP zu bewerten ist. Dabei wird herausgestellt, welche Anforderungen und Bedingungen an WU bei KBV und ÖPP geknüpft sind und wodurch Problemfelder entstehen.

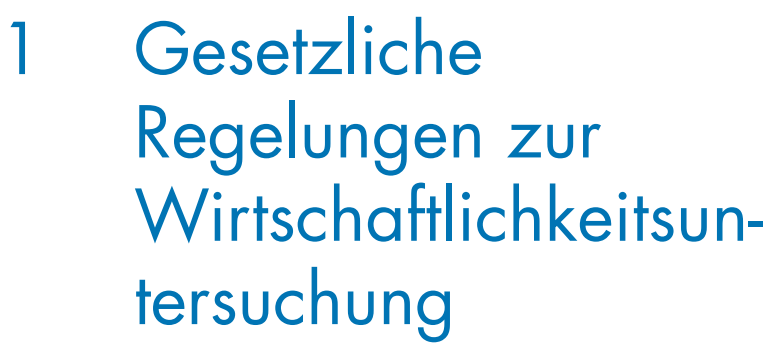

Im Gesetz über die Grundsätze des Haushaltsrechts des Bundes und der Länder Haushaltsgrundsätzegesetz (HGrG) - heißt es in $\S 6$ Abs. 2: „Für alle finanzwirksamen Maßnahmen sind angemessene Wirtschaftlichkeitsuntersuchungen durchzuführen." 37 In allen Bundesländern finden sich entsprechende Regelungen für die Kommunen. ${ }^{38}$ Doch nur die drei Stadtstaaten, Berlin, Bremen und Hamburg, schreiben konsequent für alle finanzwirksamen Maßnahmen die Durchführung von Wirtschaftlichkeitsuntersuchungen vor. Die Regelungen in den anderen Ländern offenbaren zum Teil erhebliche Interpretationsspielräume. In der Mehrheit dieser Bundesländer (Baden-Württemberg, Bayern, Brandenburg, Hessen, MecklenburgVorpommern, Niedersachsen, RheinlandPfalz, Sachsen und Schleswig-Holstein) sind nur für Investitionen von erheblicher finanzieller Bedeutung oder wie in Nordrhein-Westfalen, Sachsen-Anhalt, dem Saarland und Thüringen für Investitionen oberhalb der vom Rat festgelegten Wertgrenzen Wirtschaftlichkeitsvergleiche vorgeschrieben. Diese stellen in der Regel mehrere in Betracht kommende Beschaffungsoptionen gegenüber. ${ }^{39}$ Dabei können einerseits verschiedene Beschaffungsverfahren wie KBV und ÖPP oder andererseits unterschiedliche Beschaffungsformen wie Miete, Sanierung oder Neubau gegeneinander abgewogen werden. In fast allen Bundesländern, die auf den

\footnotetext{
Zur Entwicklung der Gemeindefinanzen siehe Anton/Diemert (2012), S. 9-23. Zur spezifischen Entwicklung der Kreisfinanzen siehe Wohltmann (2012), S. 337-374. Vgl. u. a. Beckers/Wagemann/Klatt (2013); Mühlenkamp (2012); Rechnungshof Baden-Württemberg (2009).

Gesetz über die Grundsätze des Haushaltsrechts des Bundes und der Länder - Haushaltsgrundsätzegesetz (HGrG) in § 6 Abs. 2

Vgl. die entsprechenden Landesgesetze, Baden Württemberg § 12 Abs 1 GemHVO; Bayern § 12 Abs 2 KommHV-Doppik i.V.m. §10 KommHV-Kameralistik; Brandenburg § 9 Abs. 2 GemHV; Berlin § 7 Abs. 2 LHO; Bremen § 7 Abs. 2 LHO; Hamburg § 7 Abs. 2 LHO; Hessen § 12 Abs. 1 GemHVO; Mecklenburg-Vorpommern § 9 Abs. 1 GemHVO-Doppik und § 9 Abs. 2 GemHVO; Niedersachsen § 12 Abs. 1 GemHKVO; Nordrhein-Westfalen § 14 Abs. 1 GemHVO; Rheinland-Pfalz § 10 Abs. 1 GemHVO; Saarland § 12 Abs. 1 KommHVO; Sachsen § 12 Abs. 2 SächsKomHVO-Doppik; Sachsen-Anhalt § 11 Abs. 1 GemHVO Doppik; Schleswig-Holstein § 12 Abs. 1 GemHVO-Doppik; Thüringen § 10 Abs. 1 ThürGemHV-Doppik. 
Wirtschaftlichkeitsvergleich verweisen, sollen nicht nur die Anschaffungs- bzw. Herstellungskosten, sondern ebenso die Folgekosten für die Gemeinde berücksichtigt werden. In Sachsen ist zudem "die künftige Bevölkerungsentwicklung zu berücksichtigen." 40 Eine Ausnahme der Flächenländer stellt Thüringen dar. In diesem Bundesland wird zwischen den Investitionen oberhalb der vom Gemeinderat festgelegten Wertgrenzen und denen mit erheblicher finanzieller Bedeutung unterschieden. Nur bei letzteren soll der Wirtschaftlichkeitsvergleich auf "Grundlage von dynamischen Verfahren der "Wirtschaftlichkeitsrechnung erfolgen." ${ }^{41}$ Eine ähnliche Regelung ist in der Landeshaushaltsordnung von Hamburg vorhanden, die für "geeignete Maßnahmen von erheblicher finanzieller Bedeutung" Kosten-Nutzen-Untersuchungen vorschreibt. ${ }^{42}$ Eine andere Besonderheit findet sich in der Haushaltsordnung von Berlin. Diese sieht ein Interessenbekundungsverfahren vor: "In geeigneten Fällen ist privaten Anbietern die Möglichkeit zu geben, darzulegen, ob und inwieweit sie staatliche Aufgaben oder öffentlichen Zwecken dienende wirtschaftliche Tätigkeiten ebenso gut oder besser erbringen können. ${ }^{43}$

Die wirtschaftliche Durchführung einer Infrastrukturmaßnahme ist folglich - unabhängig von der zu nutzenden Beschaffungsvariante - mit einer WU nachzuweisen. Dies gilt insbesondere, wenn diese mit erheblichen Auswirkungen auf die Finanzlage verbunden ist. In der Regel soll dies in Form eines Wirtschaftlichkeitsvergleichs geschehen. Die Realität der KBV sieht jedoch oft anders aus. Die jährlichen Prüfungsberichte der Landesrechnungshöfe offenbaren, dass Wirtschaftlichkeitsuntersuchungen und -vergleiche bei weitem nicht bei allen konventionellen Projekten der öffentlichen Hand durchgeführt werden. ${ }^{44}$ Auch die Befragung ergab, dass rund ein Viertel der Kommunen (27\%) bei konventionellen Projekten keine Wirtschaftlichkeitsbetrachtung durchführen. Mit knapp $73 \%$ gab jedoch die große Mehrheit an, konventionelle Projekte auf ihre Wirtschaftlichkeit hin zu prüfen. ${ }^{45}$

\section{Abbildung 5: Führen Sie bei der KBV im Normalfall eine WU durch?}

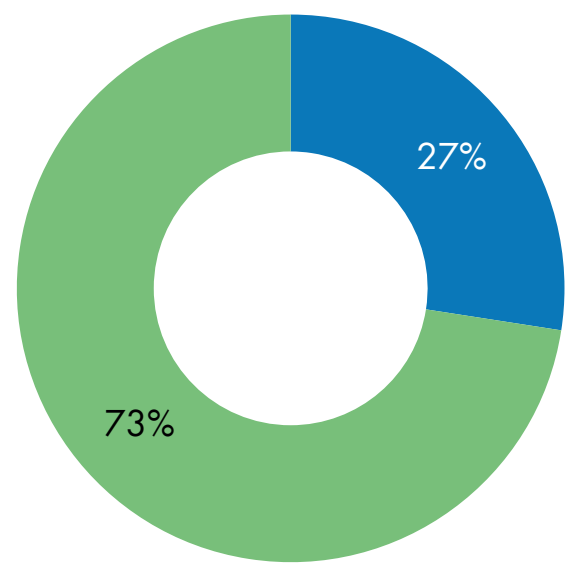

nein

ja

Quelle: Eigene Erhebung, eigene Darstellung, $n=51$.

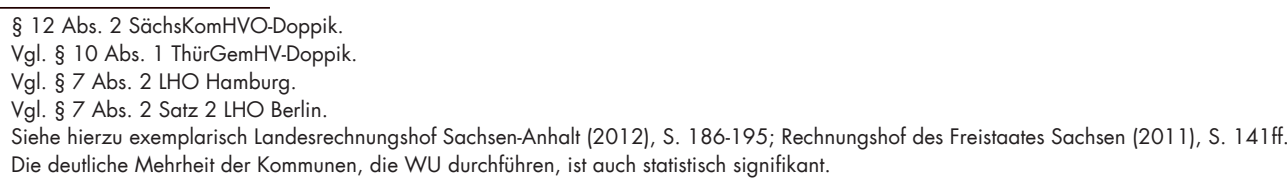


Allerdings ist bei letzteren zu hinterfragen, nach welcher Methodik diese WU durchgeführt werden. Die Kommunen besitzen nach Art. 28 Abs. 2 GG ein Selbstverwaltungsrecht, wonach sie über Angelegenheiten im eigenen Wirkungskreis selbst entscheiden können. Somit liegt die Art und Weise der Durchführung der gesetzlichen Vorgaben einer WU in der Regel im Ermessen der Kommune. Dies wird auch durch die offenen Formulierungen in den meisten Landesgesetzen deutlich, in denen "angemessene Wirtschaftlichkeitsuntersuchungen" oder die Ermittlung der wirtschafflichsten Lösung "durch Vergleich der Anschaffungs- oder Herstellungskosten und des Folgeaufwands" gefordert werden, aber das konkrete Vorgehen weitestgehend offen gelassen wird. ${ }^{46}$

ÖPP-Projekte hingegen unterliegen als kreditähnliche Rechtsgeschäfte in der Regel der Genehmigungspflicht der Rechtsaufsichtsbehörden. Von den an der Studie teilnehmenden
Kommunen mussten, wie Abbildung. 6 verdeutlicht, bis auf einen Fall alle ÖPPProjekte von der Rechtsaufsichtsbehörde genehmigt werden. Diesbezüglich ist zu berücksichtigen, dass Verwaltungsvorschriften existieren - wie exemplarisch die sächsische "VwV Kommlnvest" ${ }^{47}$ - die weitreichende Anforderungen bezüglich der Genehmigungsfähigkeit einer WU beinhalten. Eine verbindliche Wirkung haben diese in der Regel im Innenverhältnis zwischen dem Land und der unterstellten Aufsichtsbehörde. Die Art und Weise der Durchführung einer WU steht den Kommunen somit auch bei kreditähnlichen Rechtsgeschäften grundsätzlich frei. Genehmigungsfähig sind die Investitionsvorhaben in der Regel aber nur dann, wenn die WU den Anforderungen und Auflagen der Rechtsaufsicht genügt. Die Verwaltungsvorschriften und Vorgaben für die Durchführung einer WU wirken somit auch im Außenverhältnis gegenüber den Kommunen.

\section{Abbildung 6: Bedurfte das ÖPP einer Genehmigung der Rechtsaufsicht?}

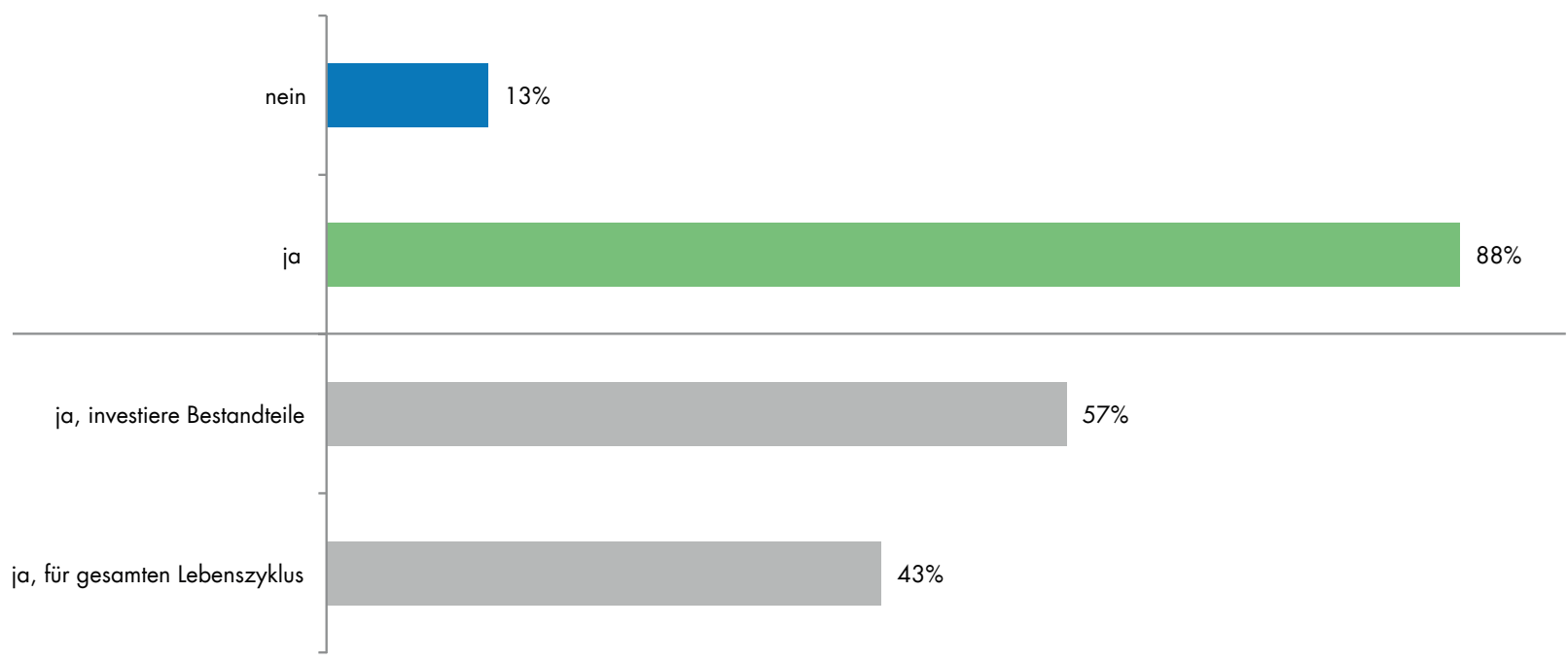

Quelle: Eigene Erhebung, eigene Darstellung, $\mathrm{n}=8$.

$46 \quad$ Vgl. die entsprechenden Landesgesetze: Brandenburg § 16 Abs. 1 KommHKV; Berlin $\S 7$ Abs. 2 LHO; Bremen $\S 7$ Abs. 2 LHO; Hamburg $\S 7$ Abs. 2 LHO; Mecklenburg-Vorpommern § 9 Abs. 1 GemHVO-Doppik; Niedersach-sen § 12 Abs. 1 GemHKVO; Rheinland-Pfalz § 10 Abs. 1 GemHVO; Saarland § 12 Abs. 1 KommHVO; Sachsen § 12 Abs. 2 SächsKomHVODoppik; Sachsen-Anhalt § 11 Abs. 1 GemHVO Doppik; Nordrhein-Westfalen und Schleswig-Holstein verweisen auf weitere gesetzliche Vorgaben; Thüringen nennt nicht näher spezifizierte dynamische Verfahren als Methode. In den Anlagen der Landeshaus-haltsordnungen gibt es allerdings umfangreiche Verwaltungsvorschriften zum Thema Wirtschaftlichkeit. Gemeinsame Verwaltungsvorschrift des Sächsischen Staatsministeriums der Finanzen und des Sächsi-schen Staatsministeriums des Inneren zur kommunal- und haushaltsrechtlichen Beurteilung von Investorenvorhaben im kommunalen Bereich. 
Bei ÖPP-Projekten wird neben den investiven Bestandteilen ebenso teilweise die Wirtschaftlichkeit des Projekts im gesamten Lebenszyklus geprüft. Im Gegensatz zur KBV (mit Ausnahme der im Abschnitt VI 1 genannten Fälle, die einen Lebenszyklusansatz verfolgen) unterliegen die bei ÖPP-Projekten vielfach im Lebenszyklus entstehenden Kosten der Genehmigung der Rechtsaufsichtsbehörde. Für die Durchführung der WU bei ÖPP-Projekten wurden nicht zuletz† deshalb in den letzten Jahren Leitfäden und Standardisierungen vorangebracht, die einen transparenten und belastbaren Vergleich von KBV und ÖPP im gesamten Lebenszyklus ermöglichen. ${ }^{48}$

\section{Ermittlung des PSC}

Der Vergleich der Wirtschaftlichkeit von KBV und ÖPP ist die entscheidende Stufe im ÖPPVerfahrensprozess, mit dem die Entscheidung für oder gegen die ÖPP-Realisierungsvariante objektiv und transparent dargestellt wird. Am Ende dieses Prozesses steht die abschließende Wirtschaftlichkeitsuntersuchung, bei der die aus einer wettbewerblichen Ausschreibung gewonnenen realen Zahlenwerte der privaten OPPP-Angebote einem Vergleichswert für die KBV gegenübergestellt werden. Dieser Public Sector Comparator (PSC) stellt die entscheidende Vergleichsgröße im ÖPP-Wirtschaftlichkeitsverfahren dar. Im Gegensatz zu den ÖPPZahlen basieren die Werte für den PSC auf Schätz- und Erfahrungswerten, die der Verwaltung aus früheren und laufenden Projekten, aber auch auf der Basis von üblichen Standardkostensätzen und Nutzungskostenkennwerten für Gebäude bekannt sind. ${ }^{49}$ Ebenso können diese durch den Rückgriff auf externe Expertise gewonnen werden.
Die Rechnungshöfe mahnen an, dass der Rückgriff auf geschätzte Zahlenwerte für einen belastbaren Wirtschafflichkeitsvergleich nicht ausreichend ist. ${ }^{50}$ Sie verweisen auf die Nutzung von ABC-Ausschreibungen. Parallelausschreibungen wie diese sind jedoch, besonders im Zusammenhang mit lebenszyklusorientieren ÖPP-Projekten, mit eigenen Schwierigkeiten verbunden. ${ }^{51}$

Bei der Ermittlung des PSC existieren noch weitere Punkte, die aus Sicht der Rechnungshöfe bei der Umsetzung bisheriger ÖPP-Projekte zu einer Verzerrung des Wirtschaftlichkeitsvergleichs führen. So ist bei ÖPP eine fundierte wirtschaftliche und juristische Analyse unabdingbar. Allerdings ist zu erwarten, dass viele Kommunen, insbesondere solche mit einer kleineren Personalausstattung, diese Expertise nicht innerhalb der Verwaltung vorhalten und demzufolge auf externe Beraterleistungen zurückgreifen. Abbildung 7 stellt dar, in welchen Bereichen des Planungs- und Entscheidungsprozesses die teilnehmenden Kommunen auf externe Berater zurückgegriffen haben. Von den Kommunen mit OPP-Erfahrungen hat sich die überwiegende Mehrheit bei der Rechtsberatung, der Bestimmung der WU und des PSC sowie bei Finanzierungsfragen extern beraten lassen. Die Darstellung zeigt aber auch, dass die Beraterkosten nur in einem Fall eine Grenze von $4 \%$ des Gesamtvolumens überschritten haben. Damit ist das finanzielle Gewicht externer Berater bei ÖPP Projekten als überschaubar einzuordnen.

\footnotetext{
$48 \quad$ Vgl. den Leiffaden der Arbeitsgruppe Wirtschaftlichkeitsuntersuchungen bei PPP-Projekten (2006) sowie das WU-Standardmodell der ÖPP-Deutschland AG.

49 Vgl. dazu die Publikationen des Baukosteninformationszentrums Deutscher Architektenkammern (BKI). Für Instandsetzungs- und Betriebskosten von Immobilien siehe Baukosteninformationszentrum (BKI)(2012).

Vgl. Präsidentinnen und Präsidenten der Rechnungshöfe des Bundes und der Länder (2011), S.18f.

Siehe dazu in dieser Studie Kapitel VI 4 Parallelausschreibung.
} 
Abbildung 7: Welche externen Beraterleistungen haben Sie in Anspruch genommen? Wie hoch beliefen sich jeweils die angefallenen Kosten in Relation zum gesamten Projektvolumen?

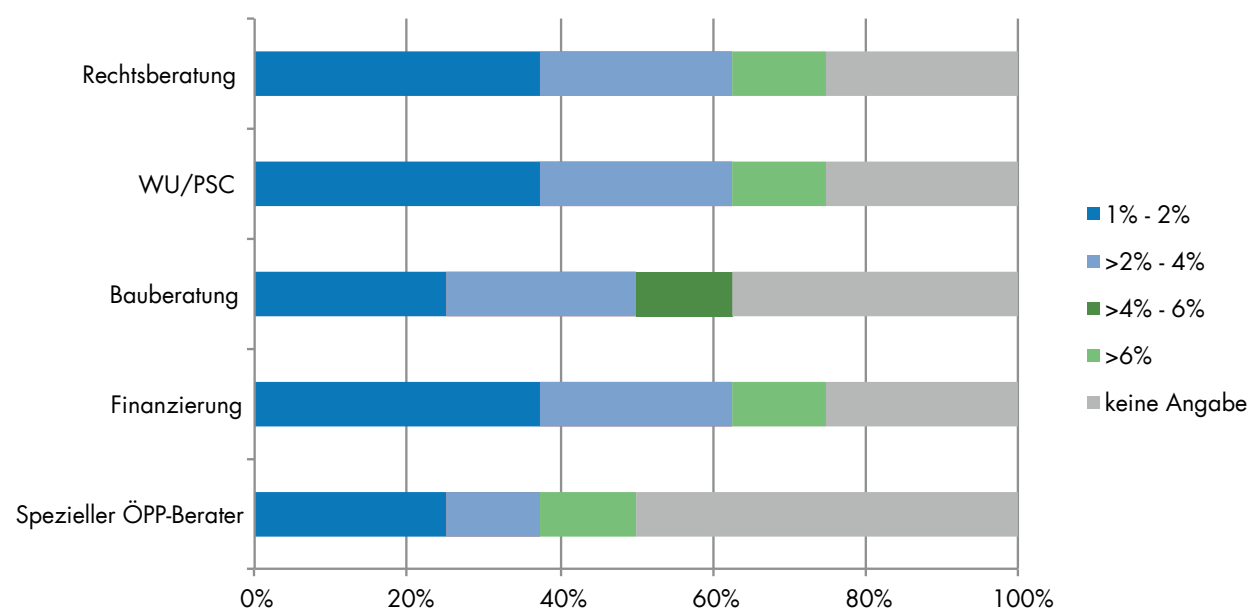

Quelle: Eigene Erhebung, eigene Darstellung, $\mathrm{n}=8$.

In externen Beratungsleistungen erkennen die Rechnungshöfe jedoch die Gefahr einer starken Eigennutzorientierung auf Beraterseite. $^{52}$ Sie argumentieren, dass verschiedene Stellschrauben existieren, an denen interessengeleitetes Handeln ansetzen und das Ergebnis der WU beeinflusst werden könnte. Dieses Problem ist in erster Linie unabhängig von der Beschaffungsvariante vorhanden. Bei der Erstellung öffentlicher Infrastrukturleistungen ist eine Vielzahl von Interessen von Verwaltung, politischen Vertretern (Räte und Fraktionen), und Finanziers sowie Nutzern und Einwohnern, die eine alternative Verwendung der öffentlichen Mittel präferieren, charakteristisch. Berater sind hier eine beteiligte Gruppe von vielen, wobei die Entscheidungen nach wie vor von der Verwaltung und den Räten getroffen werden.

Ferner müssen bei der Ermittlung des PSC auch Risiken berücksichtigt werden. So liegen bei konventionellen Projekten die in der Planung geschätzten Kosten häufig deutlich unter den am Ende tatsächlich anfallenden Kosten. Dieses Phänomen zur falschen (= übertrieben optimistischen) Kostenwahrnehmung wird auch als "optimism bias" bezeichnet ${ }^{53}$ und tritt momentan - unabhängig von der gewählten Realisierungsvariante - vor allem bei der Umsetzung von Großprojekten aufgrund politischer Planungsvorgaben auf. Kostenüberschreitungen sind nicht notwendigerweise die Regel der KBV, aber in den Prüfungsberichten der Rechnungshöfe finden sich regelmäßig Beispiele, bei denen das ursprünglich geplante Kostenvolumen nicht gehalten werden kann. ${ }^{54}$ Solche Risiken müssen im Rahmen der Erstellung des PSC monetär bewertet werden. Diese Quantifizierung unterliegt jedoch stets gewissen Spielräumen, ${ }^{55}$ die bei der Bewertung des Wirtschaftlichkeitsvergleichs nicht zu vernachlässigen sind. Hierin erkennen die Rechnungshöfe eine entscheidende Stellschraube im Vergleich zwischen KBV und ÖPP, die nach ihren Prüfungserkenntnissen oft zulasten der KBV angewandt wurde. ${ }^{56}$

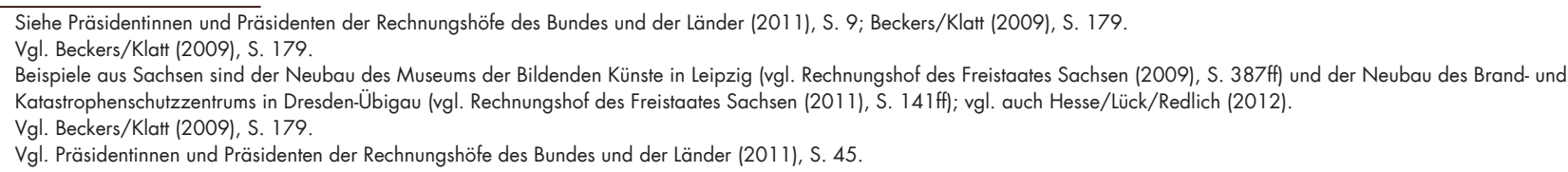




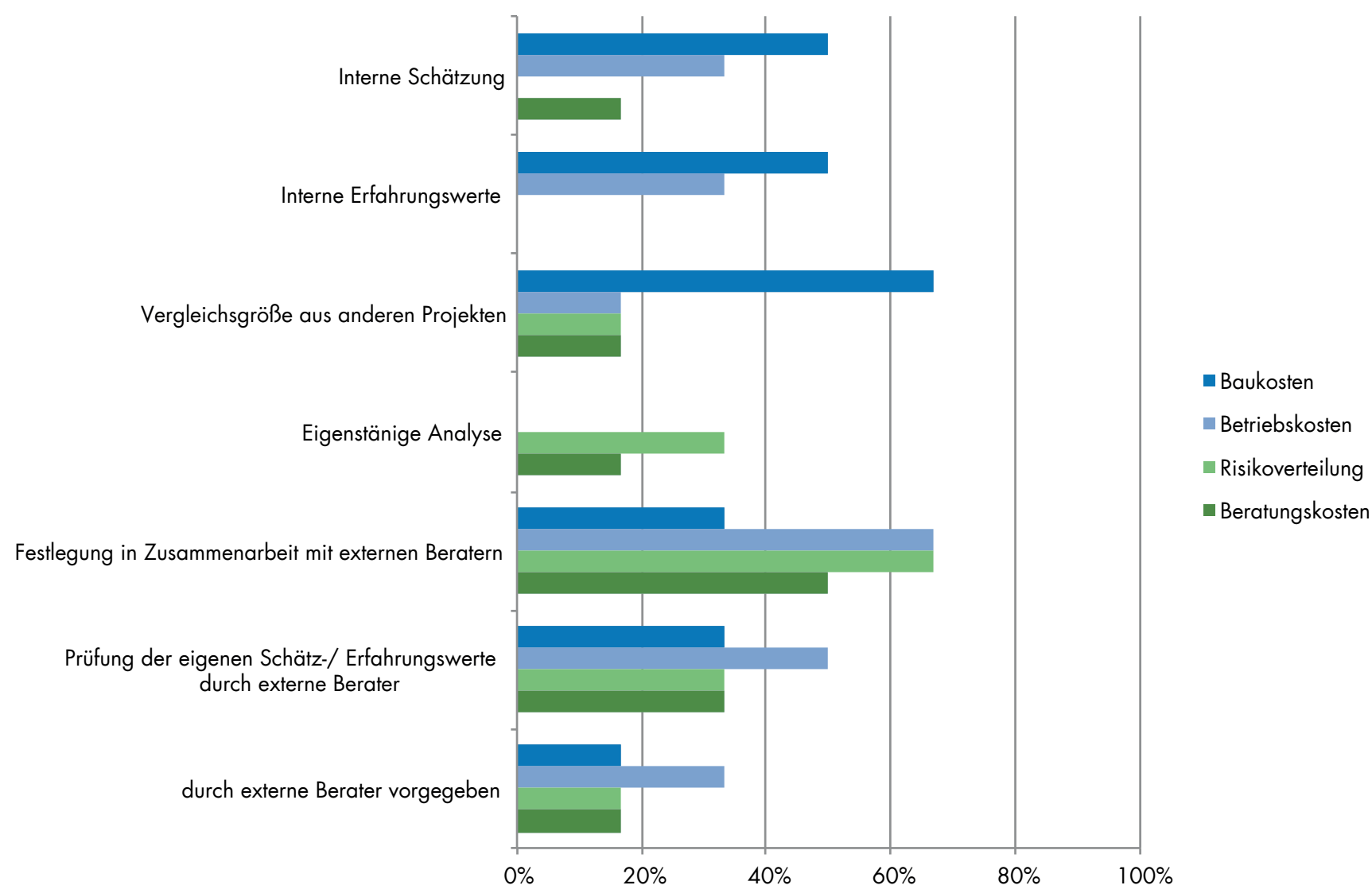

Quelle: Eigene Erhebung, eigene Darstellung, $n=6$ (Mehrfachnennungen waren möglich).

Einen kleinen empirischen Einblick über die Festlegung der Annahmen für den PSC in verschiedenen Kostenbereichen bei den teilnehmenden Kommunen, die bereits Erfahrungen mit ÖPP besitzen, zeigt Abbildung 8. Aufgrund des geringen Stichprobenumfangs lassen sich keine statistisch sicheren Aussagen ableiten, aber tendenziell kann bestätigt werden, dass die Kommunen in einem ÖPP-Verfahren den PSC-Vergleichswert nicht einfach durch den Berater vorgeben lassen. Insbesondere für den Bereich der Baukostenabschätzung können die Kommunen offenbar auf einen validen eigenen Erfahrungsschatz zurückgreifen, der mit zusätzlichen Ergänzungen der Berater weiterentwickelt wird. Allerdings wird zugleich eine Vermutung unterstützt, die bereits in einer vorangegangenen Studie des ÖPP-Kompetenzzentrums Sachsen thematisiert wurde: Die Kommunen haben augenscheinlich Schwierigkeiten, die entstehenden Folgekosten einer Investition konkret über den gesamten Lebenszyklus zu quantifizieren. ${ }^{57}$ Die Betriebskosten und die Risikoverteilung wurden daher primär mit zusätzlicher Hilfe von externen Beratern festgelegt. 


\section{Wirtschaftlichkeits- vergleich}

Der Wirtschaftlichkeitsvergleich ist bei KBV und ÖPP in einem mehrstufigen Verfahren vorgesehen. ${ }^{58}$ Dieser wird jedoch bei beiden Beschaffungsvarianten unterschiedlich gehandhabt.

Erscheint eine Projektrealisierung durch ÖPP als effizientes Mittel, wird zunächst eine vorläufige WU (vWU) erstellt. Da zu diesem Zeitpunkt noch keine vertieften Planungsunterlagen konzipiert wurden, kann es sich nur um eine Überschlagsschätzung handeln. Die Verwaltung ermittelt dabei Eckdaten des Projektes und bestimmt vor dem Hintergrund des anvisierten bzw. zur Verfügung stehenden Finanzrahmens die möglichen Qualitätsstandards. Hierfür kann auf Erfahrungswerte zurückgegriffen werden. Diese Analyse und Bestimmung des PSC muss grundsätzlich ebenso bei der KBV durchgeführt werden und sollte daher auch bei ÖPP für die Verwaltung umsetzbar sein. ${ }^{59}$

Die Kommunen, die angegeben haben, für ihre konventionellen Projekte eine WU zu erstellen, ${ }^{60}$ wurden daher näher befragt, mit welchen Instrumenten sie die Wirtschaftlichkeit der Maßnahmen nachweisen.

\section{Abbildung 9: Genutzte Instrumente des Wirtschaftlichkeitsvergleichs der KBV.}

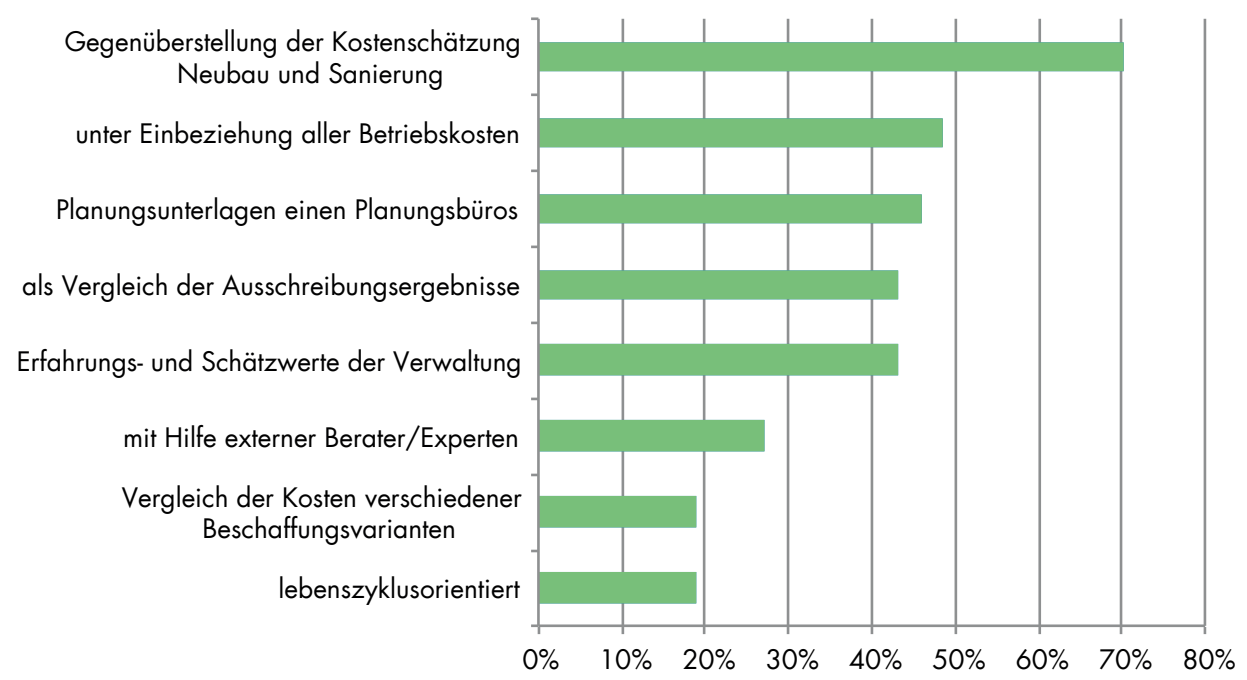

Quelle: Eigene Erhebung, eigene Darstellung, $n=37$. 
Abbildung 9 verdeutlicht, dass viele Kommunen mehrstufige Verfahren und verschiedene Instrumente einsetzen. Bei $70 \%$ der Kommunen werden die Kosten eines Neubaus der Infrastruktureinrichtung denen einer Sanierung gegenübergestellt. Dies stellt die häufigste Form eines Wirtschaftlichkeitsvergleichs dar. Allerdings wird auch die Bedeutung der Betriebsphase in vielen der antwortenden Kommunen aktiv gewürdigt. Fast die Hälfte bezieht Betriebskosten in den Wirtschaftlichkeitsvergleich ein. Allerdings bedeutet dies zugleich, dass in den übrigen $50 \%$ der Kommunen, die angegeben haben, bei ihren KBV-Projekten Wirtschaftlichkeitsvergleiche durchzuführen, die Betriebskosten in der Betrachtung keine oder nur eine untergeordnete Rolle spielen. Eine Orientierung am gesamten Lebenszyklus nimmt dagegen nur eine Minderheit (19\%) der Kommunen vor. Unter Berücksichtigung des reichlichen Viertels der Kommunen, das keinerlei WU durchführt, liegt der effektive Anteil der Kommunen, die die WU unter Berücksichtigung der Betriebsphase durchführen, bei gerade einmal rund einem Drittel.

Sanierung bzw. Neubau bei Einbeziehung der Betriebskosten werden in der überwiegenden Mehrzahl der Kommunen auf Grundlage der $K B V$ verglichen. Weniger als ein Fünftel prüft die Kosten verschiedener Beschaffungsvarianten. Die Nutzung externen Sachverstands ist allerdings durchaus üblich. In rund der Hälfte der Fälle verlassen sich die Kommunen auf die externe Zuarbeit von Planungsbüros. Weitere externe Berater und Experten werden bei ungefähr einem Viertel der Analysen einbezogen. In vielen Fällen greifen die Kommunen auf Erfahrungs- und Schätzwerte ihrer Verwaltung (43\%) zurück. Dies beinhaltet sowohl die Prüfung der Varianten Neubau/Sanierung als auch die Betrachtung der Betriebsphase. Es kann folglich durchaus davon ausgegangen werden, dass in vielen Kommunen die Abschätzung eines PSC ohne größere Probleme möglich ist (in der Stichprobe war es rund die Hälfte). Dies ist allerdings keinesfalls in allen Kommunen der Fall.

Zudem wird der (abschließende) Wirtschaftlichkeitsvergleich von über $40 \%$ durch einen Vergleich der Ausschreibungsergebnisse durchgeführt. Dies erscheint zunächst naheliegend, da durch das Ausschreibungsverfahren eine kostenminimale und damit kosteneffiziente Umsetzung gesichert wird. Es handelt sich beim Vergleich der Ausschreibungsergebnisse jedoch bereits um eine abschließende WU, die auch bei ÖPPVerfahren durchzuführen ist. Im Zuge der vorläufigen WU ist dieses Instrument ungeeignet, da keine inhaltlichen Spielräume zur Umsetzung mehr bestehen. Wie Kapitel VI 5 dieser Studie verdeutlicht, wird dabei hauptsächlich der Anschaffungspreis betrachtet. Andere Vergabekriterien spielen regelmäßig eine untergeordnete Rolle. Insofern handelt es sich nicht um eine Wirtschaftlichkeitsuntersuchung im eigentlichen Sinne.

In der Befragung sollte von den Kommunen, die eine WU aktiv nutzen, angegeben werden, wie stark die drei Gruppen „eigene Verwaltung", "externe Planungsbüros" und "externe Berater" typischerweise in die Erstellung einbezogen werden (Abbildung 10). Im Durchschnitt zeigt sich eine etwa hälffige Beteiligung der öffentlichen Hand und privater Berater. ${ }^{61}$ Diese Ergebnisse zeigen, dass die Einbindung privaten Knowhows nicht nur im Rahmen eines ÖPP-Projekts erfolgt, sondern ebenso bei der KBV regelmäßig darauf zurückgegriffen wird. 


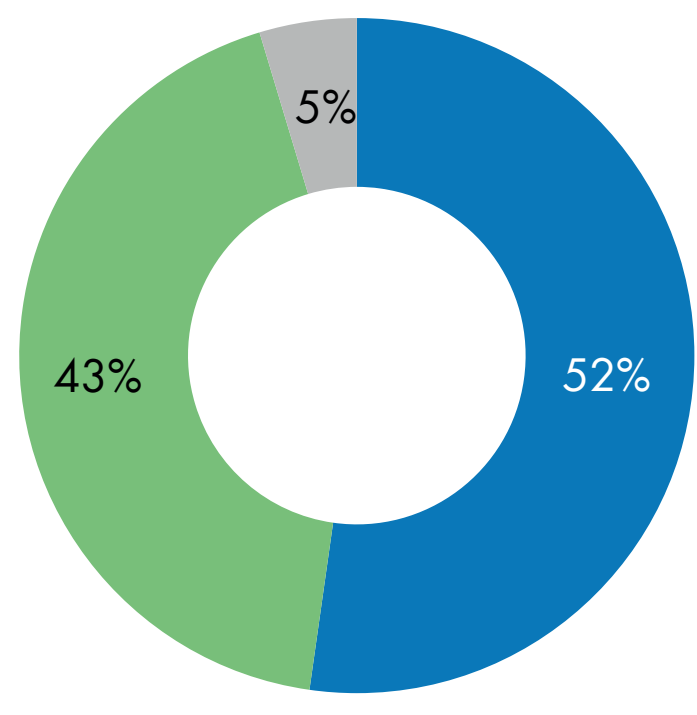

Quelle: Eigene Erhebung, eigene Darstellung, $\mathrm{n}=31$.

\author{
- Erstellung WU durch eigene \\ Verwaltung \\ Erstellung WU durch \\ externes Planungsbüro \\ Erstellung WU durch \\ externer Berater
}

Beim Wirtschafflichkeitsvergleich bei ÖPP-Projekten werden die als PSC bezeichneten Kosten der KBV denen einer ÖPP-Realisierung gegenübergestellt. Die Ermittlung der PSC-Werte erfolgt, wie bei der Wirtschaftlichkeitsuntersuchung der KBV üblich, aufgrund verwaltungsinterner Erfahrungs- und Schätzwerte sowie durch die Hinzuziehung von externen Planungsbüros, Beratern und Experten. Da trotz einer Vielzahl von kommunalen ÖPP-Projekten die meisten Kommunen mit dieser Beschaffungsvariante noch wenig Erfahrung haben, ist zur Ermittlung der (vorläufigen) ÖPP-Vergleichswerte zumeist auf das Know-how von projekterfahrenen Beratungsunternehmen zurückzugreifen. Die grundlegenden Rahmenbedingungen der ÖPP-Variante werden dabei von der
Kommune vorgegeben. Im Unterschied zum Wirtschaftlichkeitsvergleich der KBV wird bei ÖPP in der Regel von Anfang an der gesamte Lebenszyklus der anvisierten Infrastruktureinrichtung betrachtet. Neben Anschaffungskosten werden die Folgekosten für Wartung und Instandhaltung sowie die laufenden Betriebskosten analysiert. Um alle Kosten zu berücksichtigen und die Varianten sachgerecht miteinander vergleichen zu können, kann auch die Finanzierung Teil der Betrachtung sein. Ergibt die vWU einen Kostenvorteil der ÖPP-Variante, kann die Kommune sich für die Ausschreibung des Projektes als ÖPP entscheiden. Damit ist aber keinesfalls festgelegt, dass das Projekt am Ende als ÖPP realisiert wird. Die endgültige Vergabeentscheidung wird erstaufGrundlage 
der abschließenden WU getroffen.

Der Großteil der Gesamtkosten der Infrastrukturmaßnahme fällt in der Betriebsphase an. Die wichtigsten Rahmenbedingungen, die Effizienzpotentiale aufzeigen können, werden u. a. mit Ausgestaltung der technischen Anlagen, der Erarbeitung des Gebäudestandards (insbesondere energetische Standards) und der Raumgestaltung bereits in der Planungsphase gesetzt. Aus diesem Grund werden ÖPP-Projekte im Gegensatz zur KBV in der Regel funktional ausgeschrieben. Dem privaten Partner ist es so möglich, ab der Entwurfsplanung eigene Ideen und Lösungsansätze einzubringen und betriebskostenoptimiert zu planen, um eine kostenminimale Lösung bezogen auf die Gesamtkosten im Lebenszyklus zu erreichen. Bei der KBV ist dies aufgrund des Beschaffungsansatzes viel schwerer möglich. Zum einen erfolgen Planung, Bau und Betrieb durch unterschiedliche private Unternehmen. Zum anderen steht mit der Ausschreibung der Bauleistungen in vielen Bereichen noch nicht fest, wer für die späteren Betriebs- und Wartungsleistungen zuständig sein wird, denn diese Ausschreibungen erfolgen separat zu einem späteren Zeitpunkt. Innovative Lösungsansätze und Synergieeffekte, die aus der Zusammenarbeit von Planern, Facility-Managern und Baufachleuten entstehen können, bleiben deshalb bei der KBV vielfach ungenutzt. Die durch die Ausschreibung gewonnenen Ergebnisse sind deshalb zwar kostenminimal bezogen auf die Baukosten, jedoch oft nicht bezüglich der Gesamtkosten im Lebenszyklus.

Im Zuge der vertieften Planung der Projekte werden die in der vWU vorgenommen Kostenschätzungen der ÖPP-Variante durch reale Werte ersetzt. Dies wird, wie bei der KBV, mit Hilfe eines Vergleiches der in einem Ausschreibungsverfahren belastbaren Zahlen durchgeführt. Im
Gegensatz zur (abschließenden) WU der KBV, die im ersten Schritt darauf abzielt die wirtschaftlichsten Angebote für die Erstellung der Infrastruktureinrichtung zu ermitteln und erst im zweiten Schritt die Betriebsleitungen einzeln auszuschreiben und zu vergeben, liegen bei der abschließenden WU von ÖPP-Projekten verbindliche Angebote für beide Bereiche vor. Nur dadurch lassen sich die gesamten Kosten und Qualitätsniveaus aller im Lebenszyklus der Infrastruktur benötigten privaten Leistungen untereinander vergleichen, um dasinsgesamtwirtschaftlichste Angebot zu identifizieren. Dieses Vorgehen ermöglicht es, dass im Unterschied zur KBV belastbare Ausschreibungsergebnisse für den gesamten Lebenszyklus mit der Prognose des PSC verglichen werden können. Nur wenn dieser Vergleich zugunsten der ÖPP-Variante ausfällt, darf diese realisiert und damit dem wirtschaftlichsten ÖPP-Angebot der Zuschlag erteilt werden. Aus diesem Grund ist es, wie der Erfahrungsbericht der Rechnungshöfe aufzeigt, essentiell wichtig, dass der PSC an sich im Verfahren ergebende Änderungen der Rahmenbedingungen angepasst und fortgeschrieben wird. Innovative Lösungsansätze und Synergieeffekte, die aufgrund des ganzheitlichen Planungsansatzes entstehen, können allerdings nur der ÖPP-Variante angerechnet werden. Dies ist folgerichtig, da davon ausgegangen werden kann, dass die öffentliche Hand, die den PSC berechnet bzw. die für die Berechnung maßgebenden Daten liefert, alle Effizienzpotenziale ausnutzt und keine Optimierungsmöglichkeiten vorsätzlich unterlässt.

Bei der Prüfung der abschließenden Wirtschaftlichkeitsvergleiche beanstandeten die Rechnungshöfe deren Berechnung. Die Grundannahmen bezüglich 
Transaktionskosten, Risikoverteilung und Berechnung der Finanzierungsvarianten seien vielfach zulasten der KBV ausgefallen, wodurch eine ÖPP-Realisierung begünstigt wurde. ${ }^{62} \mathrm{Die}$ Differenzen der berechneten Effizienzrendite sind teilweise erheblich. In dieser Betrachtung wurde die Vermutung geäußert, dass die WU in vielen Fällen "schöngerechnet" wurden, um eine ÖPP-Realisierung überhaupt zu ermöglichen. Werden die einzelnen Prüfungsergebnisse der Rechnungshöfe analysiert, so entsteht jedoch ein differenziertes Bild. Die Abweichungen in den Berechnungen sind zwar teilweise erheblich, doch nur in drei Fällen - wobei einer nicht als ÖPP realisiert wurde - führte dies dazu, dass nach dem Ergebnis der Rechnungshöfe das Projekt nicht in Form einer ÖPP hätte durchgeführt werden dürfen. In den übrigen Fällen, bei denen von den Rechnungshöfen vergleichende Berechnungen vorgenommen wurden, bestätigten die Berechnungen der Rechnungshöfe bezüglich der Wahl der Beschaffungsvariante das Ergebnis der WU. In den weiteren angeführten Fällen haben die Rechnungshöfe aber keine eigenen Vergleichsrechnungen durchgeführt bzw. durchführen können. ${ }^{63}$ Dabei wird allgemein darauf verwiesen, dass die genauen Effizienzrenditen nicht schlüssig nachgewiesen wurden. 
Abbildung 11: Vergleich der errechneten Effizienzrendite von

Beratern/Verwaltungen und Rechnungshöfen.

\begin{tabular}{|c|c|c|c|c|}
\hline $\begin{array}{l}\text { ÖPP-Projekt } \\
\text { (Bundesland) }\end{array}$ & $\begin{array}{c}\text { Von Beratern/ Ver- } \\
\text { waltungen ermittelte } \\
\text { ÖPP-Effizienzrendite } \\
\text { (Barwerte) (in \%) }\end{array}$ & $\begin{array}{c}\text { Berechnungen der } \\
\text { Rechnungshöfe (Bar- } \\
\text { werte) (in \%) }\end{array}$ & $\begin{array}{l}\text { Differenz Effizienzren- } \\
\text { dite geringer (-) höher } \\
\qquad(+) \text { (in \%) }\end{array}$ & $\begin{array}{c}\text { Gäbe es nach den } \\
\text { Berechnungen der } \\
\text { Rechnungshöfe eine } \\
\text { Differenz der Entschei- } \\
\text { dung für oder gegen } \\
\text { ÖPP? } \\
\end{array}$ \\
\hline $\begin{array}{l}\text { Ministerium der Finan- } \\
\text { zen (BB) }\end{array}$ & 2,0 & $-6,5$ & $-8,5$ & $\mathrm{Ja}$ \\
\hline $\begin{array}{l}\text { Justizzentrum Heidel- } \\
\text { berg (BW) }\end{array}$ & 9,95 & 9,66 & $-0,29$ & Nein \\
\hline JVAt Offenburg (BW) & 1,94 & 1,67 & $-0,27$ & Nein \\
\hline $\begin{array}{l}\text { Duale Hochschule } \\
\text { Heidenheim (BW) }\end{array}$ & $-0,54$ & $-0,54$ & 0 & Nein \\
\hline $\begin{array}{l}\text { Duale Hochschule } \\
\text { Mannheim (BW) }\end{array}$ & & & - & \\
\hline $\begin{array}{l}\text { Fachhochschule Aalen } \\
\text { (BW) }\end{array}$ & 3,26 & 3,40 & 0,14 & Nein \\
\hline $\begin{array}{l}\text { Polizeirevier Ellwan- } \\
\text { gen (BW) }\end{array}$ & $-1,97$ & $-0,84$ & 1,13 & $\begin{array}{c}\text { Nein, nicht als ÖPP } \\
\text { realisiert }\end{array}$ \\
\hline $\begin{array}{l}\text { Katharinenschule in } \\
\text { der HafenCity }(\mathrm{HH})\end{array}$ & 25,76 & & - & \\
\hline $\begin{array}{l}\text { Neuorganisation im } \\
\text { Hamburger Schulbau } \\
\text { (ÖÖP) (HH) }\end{array}$ & 11,02 (nominal) & 4,83 (nominal) & $-6,19$ & Nein \\
\hline JVA Bremervörde (NI) & 5,68 & & - & \\
\hline $\begin{array}{l}\text { Berufsbild. Schulen II } \\
\text { Kaiserslautern (RP) }\end{array}$ & 4,44 & $-6,03$ & $-10,47$ & $\begin{array}{c}\text { Ja, aber nicht als ÖPP } \\
\text { realisiert }\end{array}$ \\
\hline Südbad Trier (RP) & 4,0 & $-21,50$ & $-25,5$ & $\mathrm{Ja}$ \\
\hline \multicolumn{5}{|l|}{ Schulen und } \\
\hline Kitas in Halle (ST) & 1-35 (Schulen) & & & \\
\hline 2,8-15 (Kitas) & & - & & \\
\hline $\begin{array}{l}\text { Schulen in Mag- } \\
\text { deburg Paket I (5 } \\
\text { Schulen) (ST) }\end{array}$ & 11 & & - & \\
\hline JVA Burg (ST) & 12 & & - & \\
\hline
\end{tabular}

Quelle: Darstellung in Anlehnung an Präsidentinnen und Präsidenten der Rechnungshöfe des Bundes und der Länder (2011). 
Die wirtschaftliche Durchführung aller Infrastrukturprojekte ist unabhängig von der Beschaffungsvariante transparent und belastbar nachzuweisen. Viele der von den Rechnungshöfen in diesem Zusammenhang angemahnten Defizite bei der WU von ÖPP bestehen ebenso bei der WU der KBV. In den öffentlichen Fokus geraten diese bei der KBV vielfach nicht, weil entweder gar keine WU oder kein Vergleich von unterschiedlichen Beschaffungsvarianten vorgenommen wird und damit keine Mehrkosten gegenüber einer alternativen Variante explizit ausgewiesen werden können. Der Nachweis von Risikokosten und Risikoverteilung ist vielfach nicht notwendig, da insbesondere Schnittstellenrisiken hauptsächlich von der öffentlichen Hand getragen werden. Ein zentraler Punkt der WU bei ÖPP ist die Lebenszyklusbetrachtung. Diese wird bei der KBV nur unzureichend einbezogen. Weniger als die Hälfte der Kommunen, die bei der Befragung angegeben haben, eine WU durchzuführen, beziehen die Betriebskosten in ihre WU ein. Dies deckt sich mit den Erkenntnissen aus anderen empirischen Erhebungen. ${ }^{64}$ In der Folge kann es bei konventionell durchgeführten Projekten zu Kosten- und Bauzeitüberschreitungen kommen, die immer wieder von den Rechnungshöfen angemahnt werden. ${ }^{65}$ Insgesamt steht die WU bei der KBV im Vergleich hinter der von ÖPP zurück. Die Ergebnisse dieser Studie unterstreichen jedoch ebenso, dass Wirtschaftlichkeitsvergleiche grundsätzlich bei allen Beschaffungsvarianten mit Schwierigkeiten verbunden sind. Zur deren Lösung schlagen die Rechnungshöfe als Verfahren die ABC-Ausschreibung als eine Form der Parallelausschreibung vor. ${ }^{66}$ Diese wird im Folgenden näher betrachtet.

\section{Parallelausschreibung}

In ihrem Erfahrungsbericht kritisieren die Rechnungshofvertreter, dass bei der Berechnung des PSC oft nur auf Kostenkennund Schätzwerte bereits abgeschlossener Projekte zurückgegriffen wird. Aus Sicht der Rechnungshöfe ist so kein belastbarer Wirtschaftlichkeitsvergleich möglich. Sie fordern die Verwendung realer Zahlenwerte für die WU. Dies soll durch die Nutzung von ABC-Ausschreibungen als eine Form der Parallelausschreibung erreichtwerden, da hier nach Ansicht der Rechnungshofvertreter der Lebenszyklusgedanke nicht ausgeschlossen wird. ${ }^{67}$

Bei einer ABC-Ausschreibung werden die drei Realisierungsvarianten Bau (Los A), Finanzierung (Los B) sowie Bau \& Finanzierung (Los C) ausgeschrieben. Diese Form der ABC-Ausschreibung ist als "Thüringer Modell" bekannt. Durch einen Vergleich der unterschiedlichen Varianten kann die ausschreibende Verwaltung die wirtschaftlichste Angebotskombination identifizieren. Dabei stehen ihr folgende Wahl- und Verknüpfungsmöglichkeiten zur Verfügung: Entweder vergibt sie allein das Bauvorhaben an den besten Anbieter aus Teil A und verbindet dieses mit einer eigenen Finanzierung über den Kommunalhaushalt. Die Verwaltung kann aber auch die beiden besten Angebote aus Teil A (Bauleistungen) und Teil B (Finanzierungsleistungen) kombinieren. Bildet das Investorenvorhaben aus Teil $C$ das wirtschaftlichste Angebot aller Verknüpfungsoptionen, so wird ihm der Zuschlag erteilt. 
Die Leistungen des Bauvorhabens werden dabei in der Regel sowohl unter $A$ als auch unter $C$ für Generalunternehmer ausgeschrieben. ABC-Ausschreibungen sind deshalb geeignet, Finanzierungsvarianten gegenüberzustellen. Bei den ÖPP-Projekten der "ersten Generation", die insbesondere auf die Integration von Bau- und Finanzierung abzielten und den Betrieb vernachlässigten, haben sich ABC-Ausschreibungen nach Ansicht des Rechnungshofs BadenWürttemberg zur Identifizierung der geeigneten Finanzierung durchaus bewährt. ${ }^{68}$ Dieses Ausschreibungsmodell bedeutet für die öffentliche Hand jedoch einen enormen Mehraufwand, der u. a. dadurch entsteht, dass alle erhaltenen Angebote transparent verglichen werden müssen. ${ }^{69}$

Um eine belastbare Ermittlung realer Zahlenwerte des PSC für einen Wirtschafflichkeitsvergleich zu ermöglichen, muss die $A B C$-Ausschreibung jedoch ebenso die Fachlosevergabe berücksichtigen. Im Teil A müsste somit eine Aufspaltung in eine Fachlose- (A1) und eine GU-Variante (A2) vorgenommen werden. Im diesem Fall würde die Planung für die Ausschreibung bis zur Leistungsphase 5 der Honorarordnung für Architekten und Ingenieure (HOAl), der Ausführungsplanung, erarbeitet werden, da erst ab dieser Planungstiefe eine Fachlosevergabe durchgeführt werden kann. Die Leistungsphase $5 \mathrm{HOAl}$ beinhaltet die zeichnerische Darstellung des Objekts mit allen für die Ausführung notwendigen Details. Effizienzpotentiale und Synergieeffekte für einen GU bestehen dabei in der Schnittstellenkoordination. Anreize, innovative Lösungsansätze zu erarbeiten und betriebskostenoptimiert zu planen, bestehen jedoch kaum.

Die Betriebsleistungen werden bei der
ABC-Ausschreibungsvariante nicht oder nur als Nebenangebote berücksichtigt, wodurch eine lebenszyklusorientierte Betrachtung und ein entsprechender Vergleich der Angebote nicht gewährleistet werden. Doch nur damit können wirtschaftliche Lösungskonzepte gefunden werden, die die gerade in Bezug auf die gesamte Vertragslaufzeit sehr kostenintensive Phase des Betriebs optimieren. Für die lebenszyklusorientierten ÖPP-Projekte (ÖPP-Projekte der zweiten Generation), ${ }^{70}$ die seit den letzten zehn Jahren den Markt dominieren, ist jedoch gerade die Berücksichtigung der Betriebsleistungen während der Planungsphase zur Ausschöpfung vorhandener Optimierungspotenziale elementar. Durch eine frühzeitige Einbindung eines privaten Partners in die Planung - zumeist bereits ab Leistungsphase $3 \mathrm{HOAl}$ - erhält dieser im Hinblick auf den später selbst durchzuführenden Betrieb die Möglichkeitund Anreize, innovative Konzepte zu entwerfen und eine betriebsoptimierte Projektrealisierung vorzunehmen. Durch dessen Einbeziehung in möglichst viele Wertschöpfungsstufen können zudem Effizienzpotentiale und Synergieeffekte entstehen.

Darüber hinaus sind die Erfahrungen der Kommunen mit $\mathrm{ABC}$-Ausschreibungen eher gering. Dies spiegeln auch die Ergebnisse der im Rahmen der Studie befragten Kommunen wider (Abbildung 12). So haben knapp $90 \%$ noch keine ABC-Ausschreibung genutzt, während lediglich $4 \%$ auf diese Variante bereits zurückgegriffen haben. Bei diesen Kommunen liegt jedoch kein Zusammenhang zwischen der Nutzung von ABC-Ausschreibungen und ÖPP vor, da sie allesamt noch keine lebenszyklusorientierten ÖPP-Projekte durchgeführt haben.

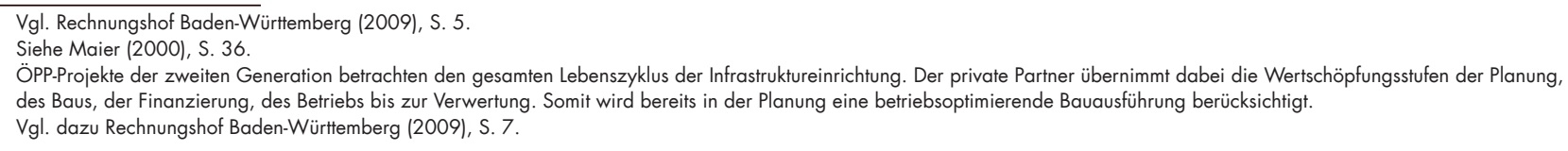




\section{Abbildung 12: Nutzung von ABC-Ausschreibungen insgesamt.}

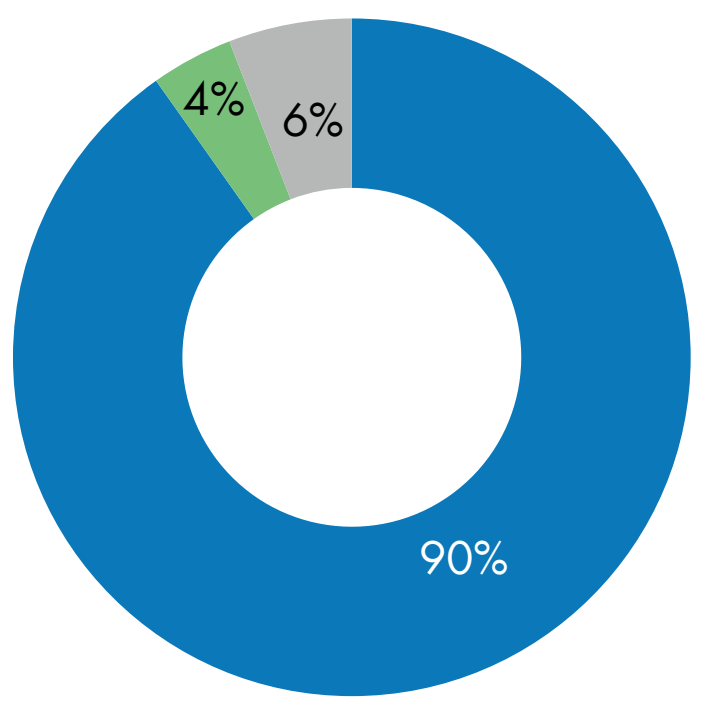

Quelle: Eigene Erhebung, eigene Darstellung, $\mathrm{n}=51$.

Aus diesen Gründen sind ABC-Ausschreibungen nicht geeignet, der Forderung der Rechnungshöfe nach belastbaren (realen) Zahlen für den Wirtschaftlichkeitsvergleich Rechnung zu tragen. Zudem ist die ABC-Ausschreibung in Form des "Thüringer Modells" für ÖPP-Projekte, die einen Lebenszyklusansatz verfolgen, nicht zweckmäßig. Für eine Berücksichtigung des Betriebs und um belastbare Wirtschaftlichkeitsvergleiche mit realen Werten zu ermöglichen, müsste die $A B C$-Ausschreibung um weitere Ausschreibungsbestandteile ergänzt werden. Die ABC-Ausschreibung in dieser Hinsicht zu modifizieren, gestaltet sich jedoch in der praktischen Umsetzung schwierig. Die dafür notwendigen ABCD- bzw. ABCDE-Ausschreibungen ${ }^{71}$ würden den Aufwand und die Transaktionskosten enorm ansteigen lassen. In der Folge wäre keine wirtschaffliche Realisierung dieser Projekte möglich.

$$
\begin{aligned}
& \text { nein } \\
& \text { ja } \\
& \text { keine Angabe }
\end{aligned}
$$

Als deutlich weniger verwaltungsaufwändige Alternativ bleibt, die Qualität der PSCSchätzwerte für die KBV weiter zU verbessern und sie zielgenauer und belastbarer zu bestimmen. An der Erreichung dieses Ziels können die Rechnungshöfe aufgrund ihrer umfangreichen und fundierten Erfahrungen aktiv mitwirken. 


\section{Vergabekriterien (Lebenszyklusansatz)}

Es wurde bereits deutlich, dass die Betrachtung unterschiedlicher Wertschöpfungsstufen (Planung, Bau, Finanzierung, Betrieb, Verwertung) den entscheidenden Punkt für die Wirtschaftlichkeit von ÖPP-Projekten im Vergleich zur KBV darstellt. Der Lebenszyklusansatz bei ÖPP-Projekten zwingt die öffentliche Hand dazu, sich bereits in der Phase der Planung über entstehende Folgekosten während des Betriebs oder über Kosten für Sanierungs- und Instandhaltungsmaßnahmen einer Infrastruktur Gedanken zu machen und diese bereits vor Baubeginn quantitativ abzuschätzen.

Eine lebenszyklusorientierte Betrachtung veranlasst auch die private Seite, die Bauleistungen betriebsoptimierend zu planen und durchzuführen. Da der private Partner das Ziel verfolgt, seine Kosten über die gesamte Vertragslaufzeit zu optimieren, wird er dazu tendieren, auf eine hohe Qualität der verwendeten Baustoffe und der Bauleistungen insgesamt zu achten, um die Kosten für spätere Sanierungs- und Instandhaltungsmaßnahmen zu minimieren sowie weitere Facility Management-Leistungen soweit wie möglich zu optimieren. Nicht zuletzt spielen hier Aspekte der energetischen Standards und deren Folgen eine Rolle. Die langfristige Bindung des privaten Partners verpflichtet ihn, aktuelle Vorgaben bei der Planung einzuhalten. ${ }^{72}$ Zum Teil werden Energieverbrauchsmengen garantiert und somit Anreize für die Nutzer gesetzt, sodass die faktische Einhaltung der Planvorgaben nicht nur in seinem eigenen Interesse, sondern auch im Nutzerinteresse liegt. Sollte die Einhaltung nicht erfolgen, muss er selbst geeignete Maßnahmen ergreifen. Während in diesem
Fall die öffentliche Hand in der Regel nach wie vor das Risiko von Preissteigerungen von Energieträgern zu tragen hat, hat sie im Fall der KBV zusätzlich das Risiko einer fehlerhaften Planung sowie das erhebliche Risiko eines vom Plan abweichenden tatsächlichen Energieverbrauchs von Kommunalseite vollumfänglich $\mathrm{zU}$ tragen. ${ }^{73}$ Durch die Verankerung von Bonus- und Malus-Regelungen ${ }^{74}$ im Vertragswerk verfügt die öffentliche Hand über einen weiteren Anreizmechanismus, der die Wahrung des Zustands der Infrastruktur auf einem hohen qualitativen Level unterstützt.

Durch die Festlegung von sachgerechten Vergabekriterien kann schon früh diesen qualitativen Aspekten Rechnung getragen werden. Um einen Überblick über die Spannweite der relevanten Vergabekriterien bei ÖPP und der konventionellen Realisierung zu gewinnen, wurden die Kommunen nach den Aspekten befragt, die sie bei der Vergabeentscheidung berücksichtigen. Die Ergebnisse für ÖPP (Abbildung 13) und die KBV (Abbildung 14) sind weitestgehend kongruent. In beiden Gruppen war der Preis bei allen Kommunen das wichtigste Kriterium. Sowohl bei den ÖPP-Projekten (57\%) als auch bei den konventionellen Realisierungen (62\%) wurde die Energieeffizienz bei der Mehrzahl der Kommunen bei der Vergabe miteinbezogen. Dies überrascht nicht vor dem Hintergrund der momentan stattfinden Prozesse im Rahmen der Energiewende und der damit einhergehenden zunehmenden Bedeutung des Themas in der Gesellschaft. ${ }^{75}$

Die Sicherung der Funktionalität bzw. Gestaltqualität sowie der Umweltschutz werden bei ÖPP-Ausschreibungen öfter miteinbezogen als bei der KBV, was die 
Einschätzung einer stärkeren Berücksichtigung von qualitativen Aspekten bei ÖPP unterstützt. Bei der Interpretation dieser Ergebnisse ist aber zu beachten, dass die öffentliche Hand bei einer fachlosen Vergabe aufgrund der dafür notwendigen Planung bereits detaillierte Weichenstellungenfür dieVergabeentscheidung gelegt hat. Funktionalität und Qualität der ausgeschriebenen Leistungen entsprechen daher den Planungsvorgaben. Angebote, die einen höheren funktionalen Wert aufweisen könnten, werden dabei stark eingeschränkt.
Erst eine die Bau- und Betriebsausführung berücksichtigende Planung, die bei ÖPP üblich ist, ermöglicht dessen Optimierung. Insofern stellt sich die Funktionalität bezogen auf die ausgeschriebenen Leistungen bis zu einem gewissen Grad praktisch automatisch ein. Angebote, die zwar möglicherweise einen höheren funktionalen Wert haben, aber nicht den Vergabeanforderungen entsprechen, werden aus dem Verfahren ausgeschlossen.

\section{Abbildung 13: Kriterien der Vergabeentscheidung bei der KBV.}

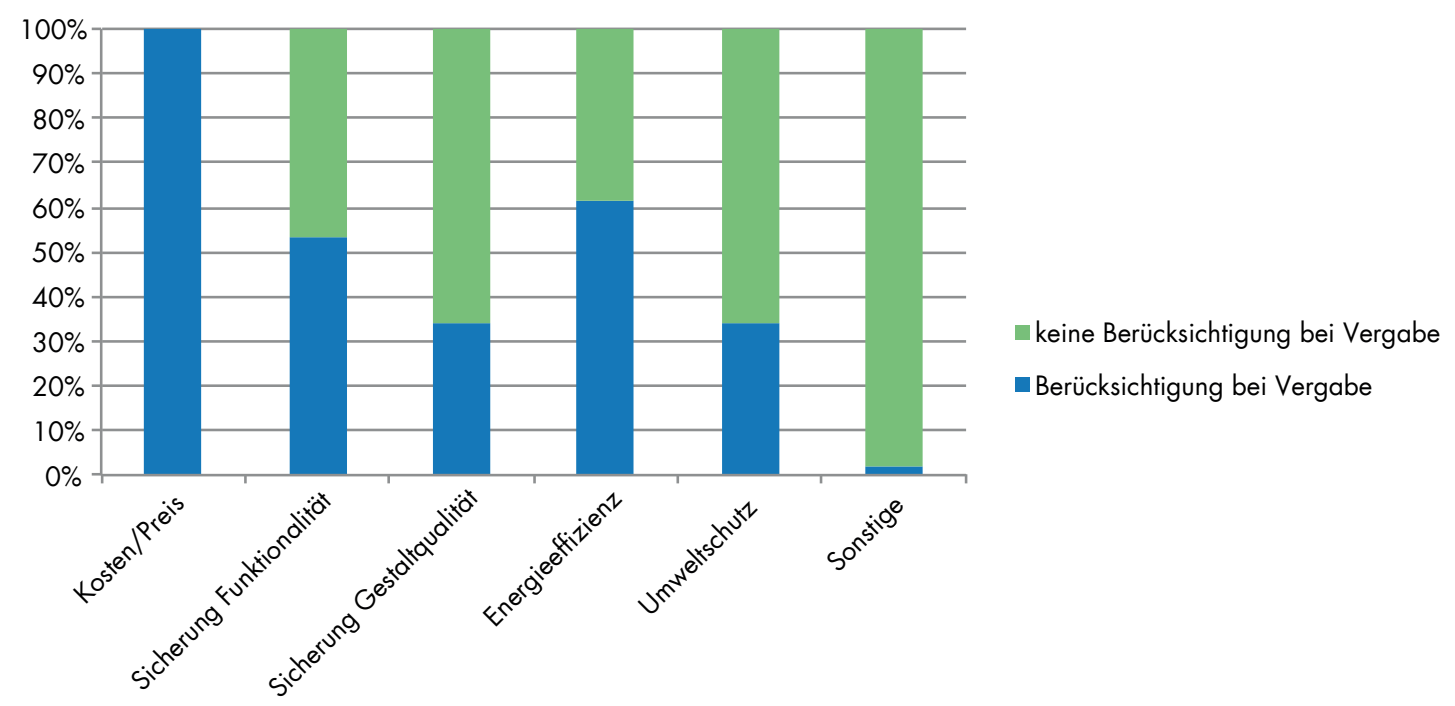

Quelle: Eigene Erhebung, eigene Darstellung, $n=47$. 


\section{Abbildung 14: Kriterien der Vergabeentscheidung bei ÖPP-Projekten.}

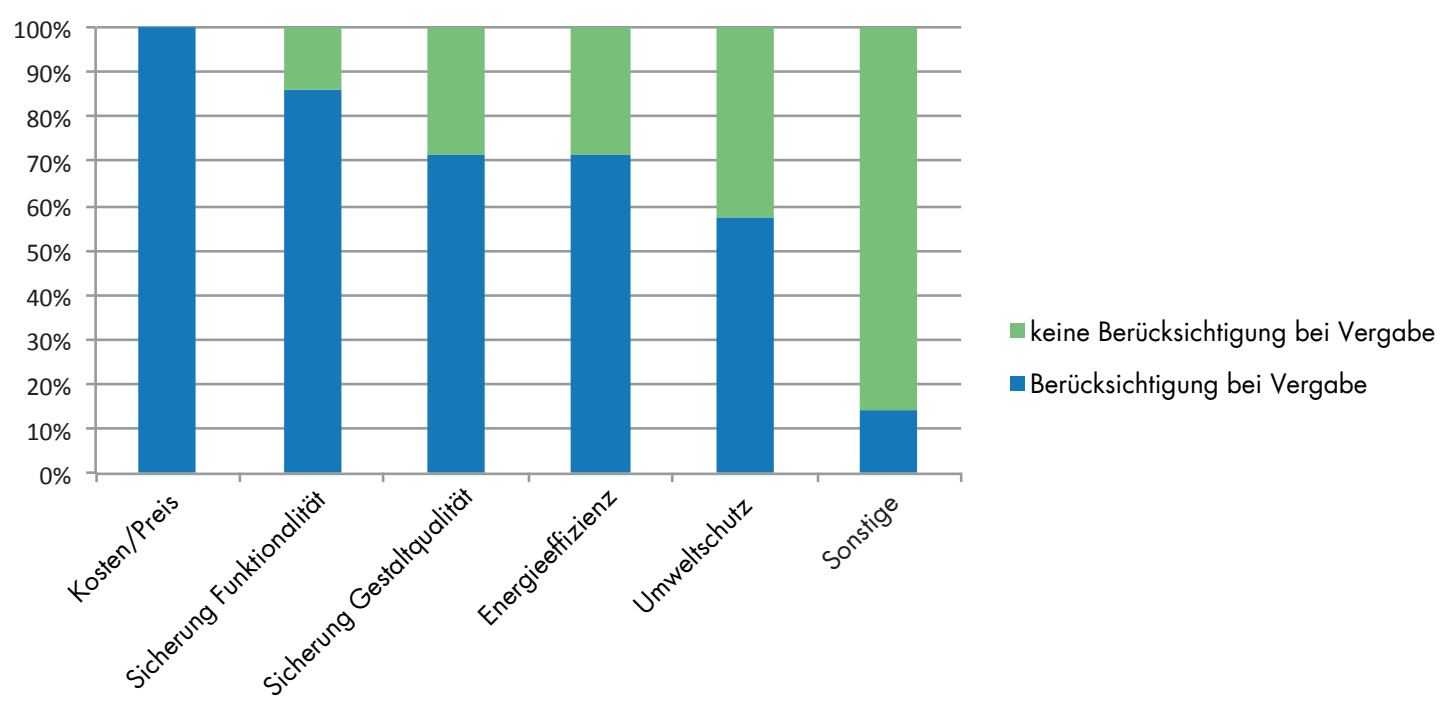

Quelle: Eigene Erhebung, eigene Darstellung, $n=7$

Ein Blick auf die tatsächliche Gewichtung der Vergabekriterien bei der KBV (Abbildung 15) offenbart, dass bei den teilnehmenden Kommunen der Preis das maßgebliche Vergabekriterium darstellt. ${ }^{76}$ Der Preis wird bei der Vergabeentscheidung durchschnittlich mit $80 \%$ gewichtet. Die Sicherung der Funktionalität sowie die Energieeffizienz haben ein deutlich geringeres Gewicht und ergänzen die Beschaffung nach dem Preis als untergeordneter Faktor bzw. im Einzelfall. Die Sicherung der Gestaltqualität sowie der Umweltschutz (über die gesetzlichen Vorgaben hinaus) haben ein kaum nennenswertes Gewicht bei der Vergabeentscheidung. 


\section{Abbildung 15: Durchschnittliche Gewichtung der Vergabekriterien bei der KBV.}

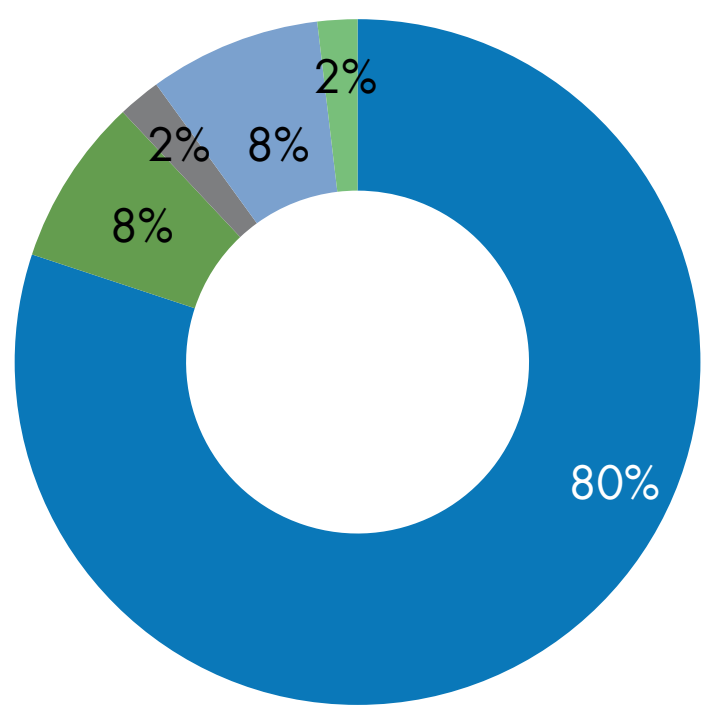

Quelle: Eigene Erhebung, eigene Darstellung, $n=47$.
- Kosten/Preis

- Sicherung der Nutzung/Funktionalität

- Sicherung der Gestaltqualität

Energieeffizienz

- Umweltschutz

\section{Einschätzung der Projektrisiken}

Die optimierte Aufteilung der Risiken zwischen der öffentlichen Hand und dem privaten Partner wird oft als ein Argument ins Feld geführt, das die Vorteilhaftigkeit des ÖPP-Ansatzes gegenüber der konventionellen Realisierung unterstreichen soll. Die Aufteilung erfolgt dabei nach der Maßgabe, dass jeder Partner die Risiken trägt, bei denen er die Wahrscheinlichkeit des Risikoeintritts und die eventuellen Risikofolgen am besten beeinflussen kann. Vor diesem Hintergrund ist es gängige Praxis, dass Planungs-, Bau- und Inbetriebnahmerisiken (z. B. Terminüberschreitungen, Mängel bei der baulichen Qualität) vom privaten Partner getragen werden. Die Risikoübernahme durch den Privaten wird über die Einpreisung der kalkulatorischen Risikokosten vollzogen. Insofern ist es wichtig, beim Vergleich der KBV mit der ÖPP-Variante für beide Verfahren eine Bewertung der projektrelevanten Risiken vorzunehmen.

In ihrem Erfahrungsbericht kritisieren die Rechnungshöfe, dass die Bewertung der Risiken oft auf einer unzureichenden Informationsgrundlage basiert und diese daher qualitativ mängelbehaftet sind. Durch dieses Vorgehen sind insbesondere die Risiken für die KBV nur vage prognostizierbar. ${ }^{77}$ In diesem Zusammenhang stellt sich die Frage nach den Gründen für die unzureichende Informationsgrundlage auf kommunaler Seite. Die Vermutung liegt nahe, dass diese risikorelevanten Daten oft nicht erhoben bzw. nicht in ausreichendem Maße ausgewertet werden. Vor dem Hintergrund dieser 
Überlegungen wurden die Kommunen gefragt, ob sie bei der konventionellen Realisierung in der Regel Risikoanalysen durchführen. Die erhaltenen Antworten unterstützen die oben genannte Annahme (Abbildung 16). $78 \%$ der Kommunen gaben an, keine Risikoanalyse im Falle der konventionellen Beschaffung zu erstellen. Die Kommunen, bei denen eine Risikoanalyse durchgeführt wird (18\%), stützen sich bei deren Erstellung meistens auf verwaltungsinterne Schätzwerte und externe Berater.

\section{Abbildung 16: Durchführung von Risikoanalysen im Rahmen der KBV.}

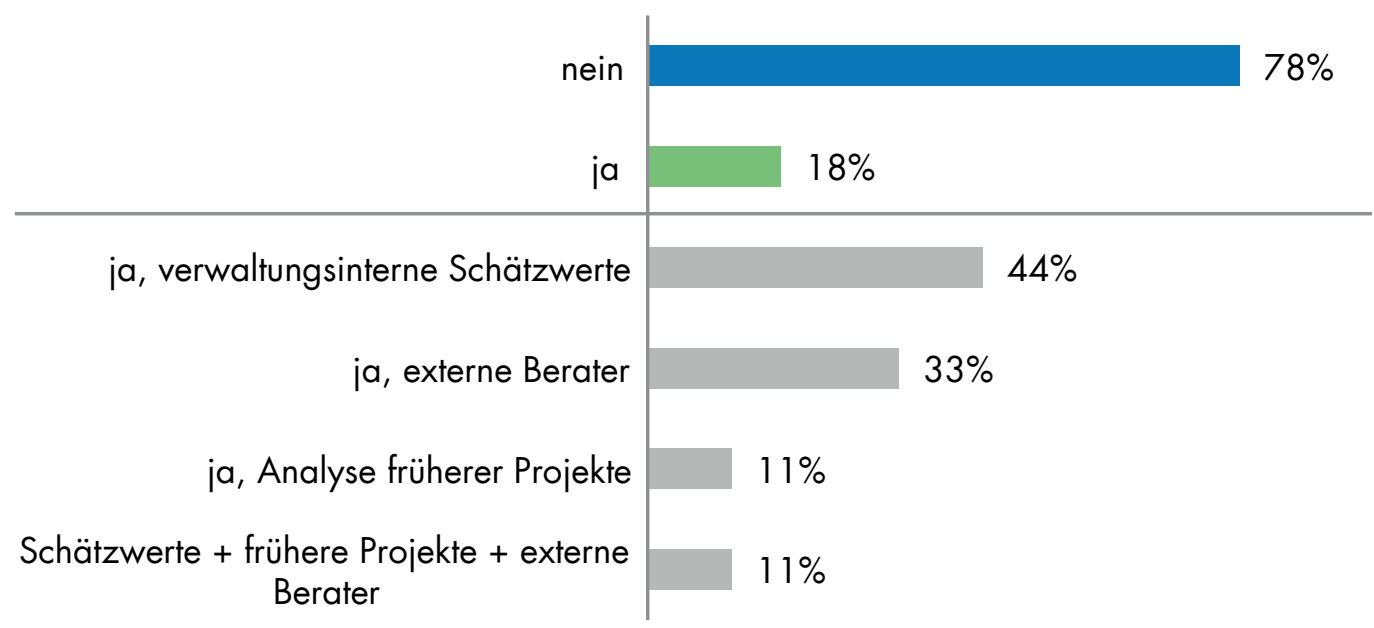

zu $100 \%$ fehlende Werte $=$ "keine Angabe"

Quelle: Eigene Erhebung, eigene Darstellung, $n=49$.

Die Betrachtung von Risiken ist aufgrund der Risikoteilung zwischen öffentlichem und privatem Partner ein wesentliches Merkmal des ÖPP-Prozesses. Es kann festgestellt werden, dass bei der WU im Rahmen von ÖPP-Projekten deutlich häufiger Risikoanalysen zum Einsatz kommen, welche sich sowohl auf die KBV als auch auf die ÖPP-Variante beziehen. Die
Unterschiede sind so groß, dass sie trotz der geringen Stichprobenumfänge als statistisch gesichert angesehen werden können. ${ }^{78}$ 


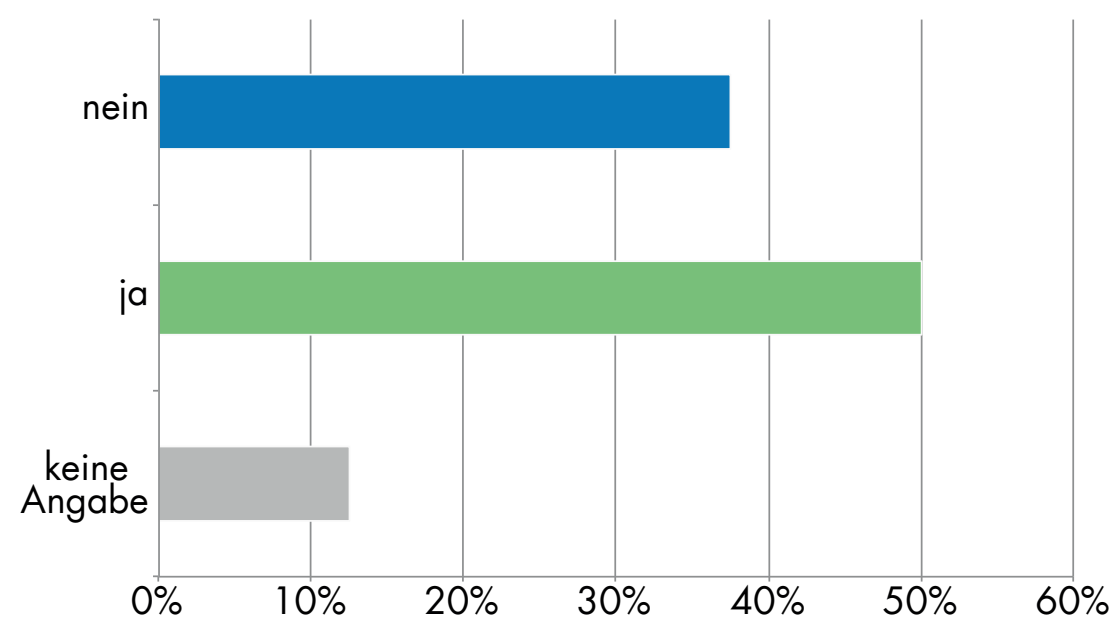

Quelle: Eigene Erhebung, eigene Darstellung, $n=8$.

\section{Transparenz und bürgerschaftliche Beteiligung}

Das Thema Transparenz besitzt in der öffentlichen Debatte über ÖPP eine große Relevanz. ÖPP-Kritiker beanstanden insbesondere die Nichtveröffentlichung von Verträgen, sodass eine Bewertung der Wirtschaftlichkeit eines PPP-Projekts nicht objektiv durch die Öffentlichkeit nachvollzogen werden kann. Die Kritik basiert vor allem darauf, dass in der vWU auf die Berechnungen der öffentlichen Hand für den PSC pauschale Zu- und Abschläge zur Bestimmung der ÖPP-Vergleichswerte erhoben werden. Dabei wird vielfach argumentiert, dass nur durch diese eine maßgeblich höhere Wirtschafflichkeit des ÖPP-Ansatzes im Vergleich zur KBV erreicht wird. ${ }^{80}$

Eine Optimierung der Transparenz in Wirtschaftlichkeitsuntersuchungen sollte ein ständiges Ziel sein. Da es sich um öffentliche Projekte handelt, werden damit ebenso die demokratischen Strukturen gestärkt. Allerdings kann eine größere Transparenz durchaus mit Nachteilen verknüpft sein. Die Offenlegung der Zahlen kann die Effizienz des Bieterwettbewerbs einschränken und einige Wettbewerber dazu veranlassen, nicht alle innovativen Lösungsvorschläge anzubieten. ${ }^{81}$ Nichtsdestotrotz ist Transparenz ein wichtiges Gut in demokratischen Gesellschaften, welches das Vertraven in das Handeln der öffentlichen Hand maßgeblich beeinflusst und somit im größtmöglichen Maße sichergestellt werden sollte, vor allem, wenn es sich um öffentliche Mittel handelt. In diesem Zusammenhang gibt es bei ÖPP eine Transparenzinitiative, in deren Rahmen einige Projektverträge veröffentlicht wurden. ${ }^{82}$

\footnotetext{
Transformation der Frage "Wurden ÖPP und die konventionelle Realisierung ohne Berücksichtigung von Risiken verglichen?".

Siehe dazu in dieser Studie Kapitel VI2 Ermittlung des PSC. Vgl. auch u. a. Präsidentinnen und Präsidenten der Rechnungshöfe des Bundes und der Länder (2011), S. 11.

Siehe Beckers/Wagemann/Klatt (2013), S. $178 \mathrm{f}$.

Siehe dazu http://www. partnerschaften-deutschland.de/transparenzplattform.
} 


\section{Einbeziehung und Information der Öffentlichkeit}

Das Thema Transparenz wird in Ansätzen im Gemeinsamen Erfahrungsbericht der Rechnungshöfe aufgegriffen. In diesem wird festgestellt, dass WU in einzelnen Fällen erst nachträglich erstellt wurden. ${ }^{83} \mathrm{Im}$ Bericht wird empfohlen, für die Vergabeentscheidung die verantwortlichen Entscheidungsgremien frühestmöglich $\mathrm{zu}$ informieren und für eine bessere Aussagekraft die Ergebnisse der WU nicht nur in relativen, sondern auch in absoluten Zahlenwerten anzugeben. ${ }^{84}$ In der öffentlichen Kritik an ÖPP wird dabei immer wieder angemerkt, dass insbesondere die entscheidungsrelevante Wirtschaftlichkeitsuntersuchung und die notwendigen Verträge zwischen privatem Unternehmen und der öffentlichen Hand schwer zugänglich sind. ${ }^{85}$ Die Zurückweisung der Forderung nach Offenlegung von Vertragsinhalten durch die privaten Unternehmen mit der Begründung, Geschäftsund Betriebsgeheimnisse wahren zu wollen, wurde scharf kritisiert. ${ }^{86}$ Darüber hinaus spielt im Zusammenhang mit der Transparenzkritik an ÖPP immer wieder eine von den Kritikern als mangelhaft betrachtete, allgemeine Information der weiteren Öffentlichkeit über die Projekte eine entscheidende Rolle. Werden, wie im Bericht der Rechnungshöfe, die Projekte isoliert dargestellt, ist eine gewisse Intransparenz zu erkennen, die dem ÖPP-Ansatz geschuldet scheint. In keiner der in der vorliegenden Studie antwortenden ÖPP-Kommunen wurden die Verträge veröffentlicht. Den Bürgern sind bei diesen nur selten Mitentscheidungs- und Partizipationsmöglichkeiten eingeräumt worden. Architektur- und Raumkonzepte sowie detaillierte Projektinformationen standen in wenigen Fällen zur öffentlichen Einsicht. Es stellt sich jedoch die Frage, ob ein mögliches Defizit an Transparenz allein ein ÖPP-immanentes Problem darstellt. 


\section{Abbildung 18: Information der Bürger bei ÖPP-Projekten.}

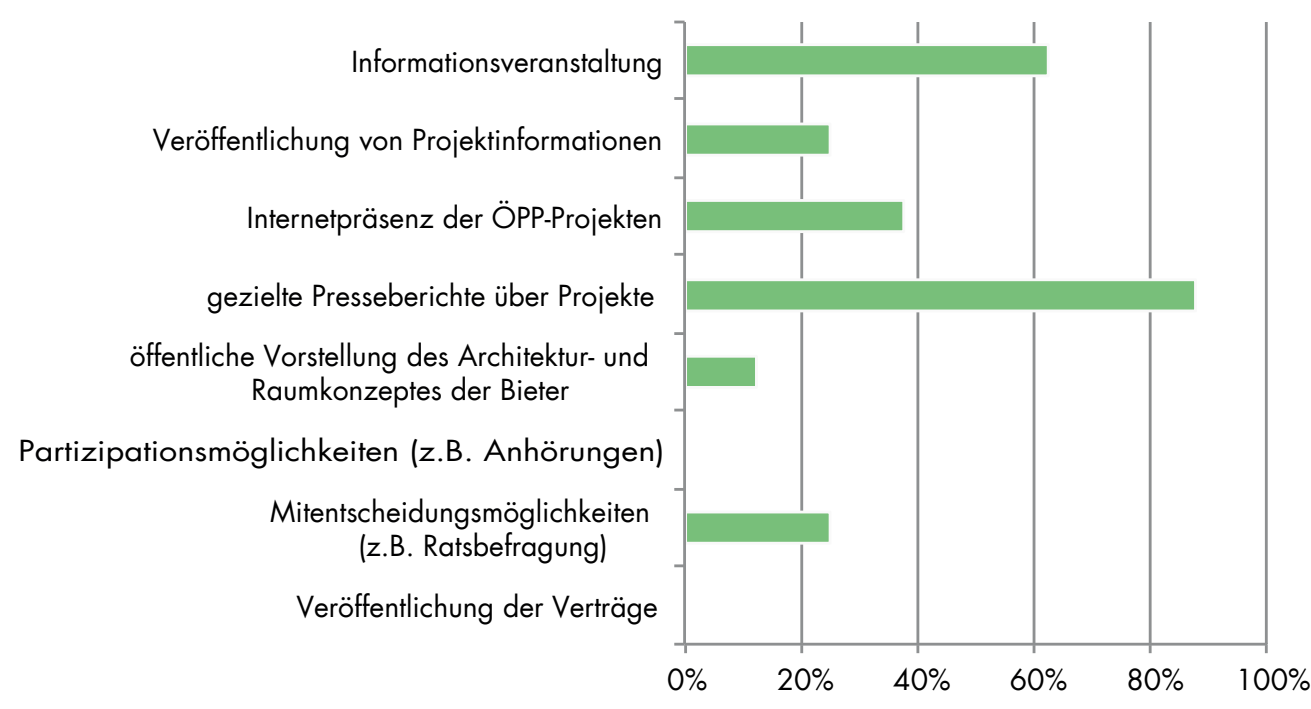

Quelle: Eigene Erhebung, eigene Darstellung, $\mathrm{n}=8$.

Der Vergleich der Befragungsergebnisse bei den ÖPP-Projekten mit den Antworten aller Kommunen zur Partizipation und Information der Bürger bei konventionellen Projekten zeigt, dass die angemahnte Verfahrensintransparenz eine Herausforderung bei allen öffentlichen Beschaffungsvarianten darstellt.

Abbildung 19 zeigt, dass die mit privaten Unternehmen geschlossenen Verträge bei konventionellen Projekten bei über 70 \% der teilnehmenden Kommunen nie oder nur selten veröffentlicht werden. ${ }^{87}$ Ebenso selten oder nie stellen jeweils rund $60 \%$ über die Ausschreibungsunterlagen hinausreichende Projektinformationen zur Verfügung oder richten eine Webseite zum Projekt ein (61\%). Wie die Befragung ergeben hat, findet eine direkte
Einbindung der Bürger und Nutzer bei konventionellen Projekten eher selten statt. Zwar geben immerhin fast ein Viertel der Kommunen an, dass sie Partizipationsmöglichkeiten bieten, doch bleiben diese häufig auf Informationsveranstaltungen und gesetzliche Anhörungspflichten beschränkt. ${ }^{88}$ In den ÖPP-Kommunen wurde die Öffentlichkeit fast immer durch Medienberichte gezielt über die ÖPP-Projekte informiert. Dabei ist zu berücksichtigen, dass ÖPP-Projekte in der öffentlichen Wahrnehmung stärker polarisieren und dass deshalb über diese regelmäßig auch intensiv berichtet wird. Vielfach werden diese dabei in der Medienberichterstattung kritisch gewürdigt. ${ }^{89}$ Bei der konventionellen Realisierung werden bei den befragten Kommunen bei 80 \% der Projekte regelmäßig Die existierenden Abweichungen zu den Befragungsergebnissen der ÖPP-Kommunen sind der geringen Fallzahl geschuldet. In einigen Kommunen werden ebenso die ÖPP-Verträge
veröffentlicht. Siehe dazu genauer die Transparenzplatfform der ÖPP Deutschland AG. Bislang (Stand Februar 2013) sind die Verträge von neun ÖPP-Projekten veröffentlicht worden http://www.partnerschaften-deutschland.de/oepp-markt/transparenzplattform/.

88 Zur Verwendung verschiedener informeller Beteiligungsverfahren bei Infrastrukturprojekten vgl. Albrecht/Grütner/Lenk/Lück/Rottmann (2012)

89 Vgl. u. a. Tempel (2010), Simantke (2013). 
- d. h. immer oder zumindest oft - Presseberichte verfasst. Ebenso werden für die Bürger in der Mehrzahl der konventionellen und ÖPPProjekte Informationsveranstaltungen durchgeführt. Beides scheint aber nicht zwingend der Fall zu sein. In Bezug auf den Wirtschaftlichkeitsvergleich bzw. die Wirtschaftlichkeitsuntersuchung wird deutlich, dass bei der KBV die Ratssitzungen zu diesem Thema in jeder fünften Kommune nicht öffentlich sind. In knapp einem weiteren Fünftel der Kommunen wird gelegentlich die Öffentlichkeit zugelassen. Demgegenüber steht, dass ebenso $40 \%$ der Kommunen mitteilten, oft oder sogar immer öffentliche Ratssitzungen zur Wirtschaftlichkeit durchführen. Diesbezüglich ist allerdings nochmals anzumerken, dass Wirtschaftlichkeitsuntersuchungen in den für die Kommunen relevanten Haushaltsvorschriften ${ }^{90}$ zwar ausdrücklich bestimmt sind, deren Ausgestaltung und Umfang aber den Kommunen selbst obliegt. Dies hat zur Folge, dass diese zum Teil faktisch gar nicht durchgeführt werden. ${ }^{91}$ Dies könnte erklären, wieso von einem relativ hohen Anteil - ein Fünftel der Kommunen keine Antwort gegeben wurde.

\section{Abbildung 19: Information der Bürger bei konventionellen Projekten.}

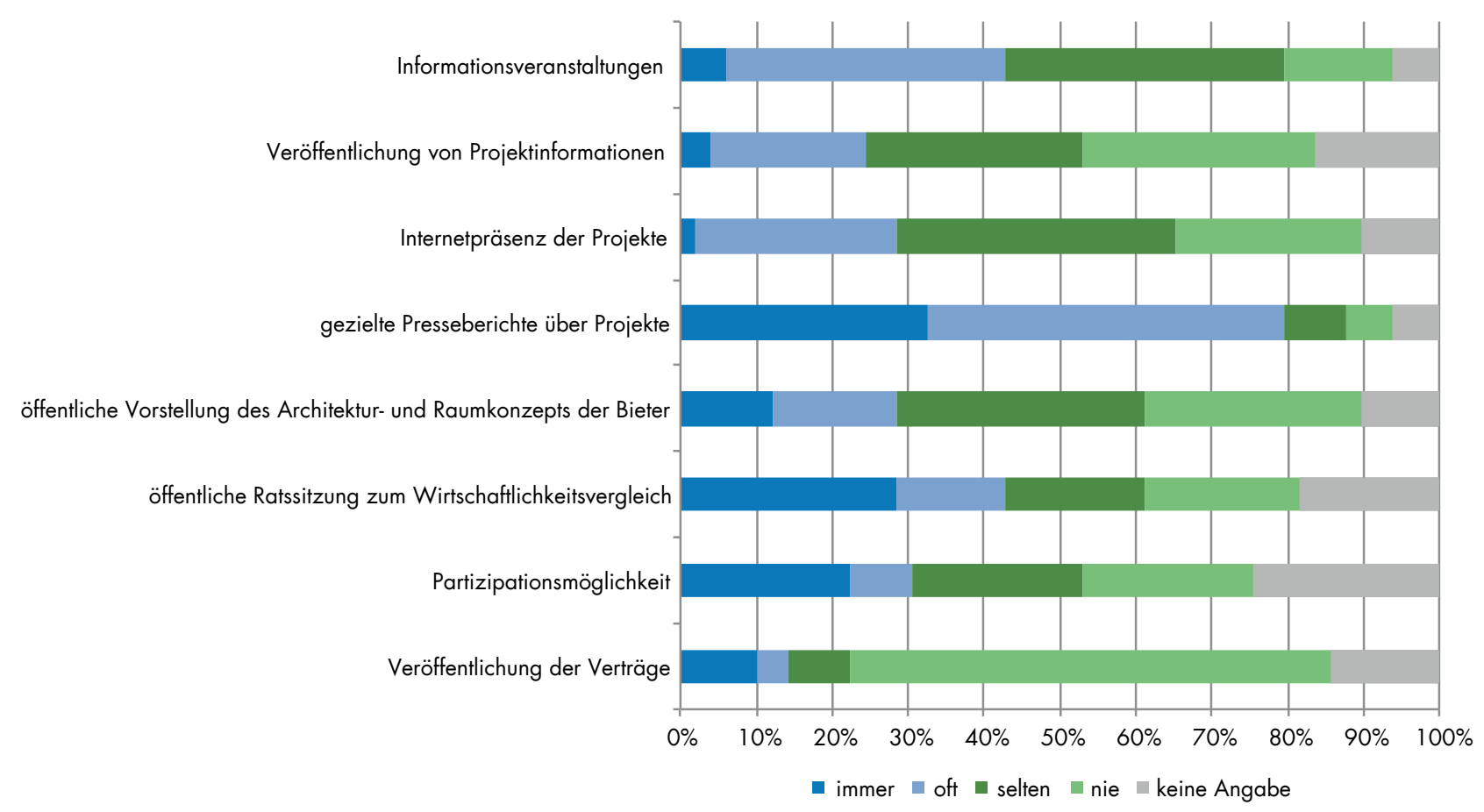

Quelle: Eigene Erhebung, eigene Darstellung, $n=46$. 


\section{Projektsteuerung}

Die öffentliche Hand befindet sich bei ÖPPProjektrealisierungen in der Position des Auftraggebers (Prinzipals) und das Unternehmen der Privatwirtschaft in der des Auftragnehmers (Agenten). Eine solche Vertragssituation ist gekennzeichnet durch konfligierende Interessenlagen. Während Politik und Verwaltung die Wahrung der öffentlichen Interessen anstreben, stellt der private Partner die eigene Gewinnerzielung in den Mittelpunkt seiner Handlungen. ${ }^{92}$ Zwischen beiden Vertragspartnern bestehen Informationsasymmetrien, die zu einseitigen Informationsvorsprüngen und Fehlanreizen führen. ${ }^{93}$ Bezogen auf ein ÖPP-Projekt bedeutet dies, dass der private Vertragspartner diese Informationsvorsprünge für sich nutzen kann, um seinen Gewinn zu maximieren. Dies könnte durch überhöhte Kostenforderungen oder die Reduzierung des eigenen Leistungsniveaus $z$. B. durch die Nutzung von qualitativ geringwertigen Baumaterialien geschehen. ${ }^{94}$

Ferner können bei dieser Vertragsausgestaltung "Hold up"-Situationen auftreten, die sich dadurch kennzeichnen, dass der Agent über weitreichende Verhandlungsmacht verfügt. Da sich der Prinzipal in einem Abhängigkeitsverhältnis gegenüber dem Agenten befindet, kann der Agent seine Verhandlungsmacht ausspielen. Diese Anreizprobleme erfordern demzufolge die Nutzung von geeigneten Governanceund Steuerungsmechanismen, die es ermöglichen, das Handeln des Agenten in adäquater Weise zu kontrollieren und ggf. zu sanktionieren. Bei ÖPP-Projekten wird versucht diese Problematik durch klar zuweisbare
Verantwortungsstrukturen, Risikoteilung und verbindliche Leistungskataloge mit vertraglichen Anreizsystemen zu minimieren. Doch auch in der KBV existiert das PrinzipalAgenten-Problem. Durch den Rückgriff auf private Kompetenz z. B. durch die Nutzung privaten Planungs-Know-hows, die privatwirtschaftliche Umsetzung der Bauleistungen oder die Durchführung der Betriebsleistungen durch private Unternehmen, liegen bei der KBV zahlreiche Abhängigkeitssituationen vor. Im Gegensatz zu ÖPP-Projekten stehen der öffentlichen Hand bei der KBV aber zahlreiche Agenten mit jeweils eigenen Interessen gegenüber. Fehlanreize, die durch die PrinzipalAgenten-Problematik entstehen, sind folglich kein alleiniges Defizit des ÖPP-Ansatzes. ${ }^{95}$ Dies wird in der Diskussion über Vor- und Nachteile dieser Beschaffungsvariante gegenüber der KBV oft vernachlässigt. Allerdings ist bei ÖPP-Projekten die "Hold up"-Problematik als schwerwiegender einzustufen, da der private Partner nicht nur einzelne Leistungen in einer Phase, sondern die Durchführungsverantwortung für das gesamte Leistungsspektrum aller übertragenen Phasen trägt. ${ }^{96}$ In gleicher Weise trägt die längere vertraglich fixierte Zusammenarbeit zu diesem Tatbestand bei. ${ }^{97}$

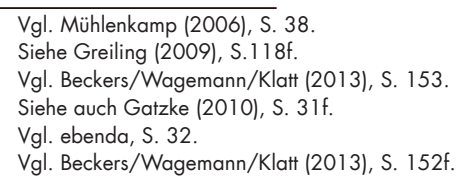




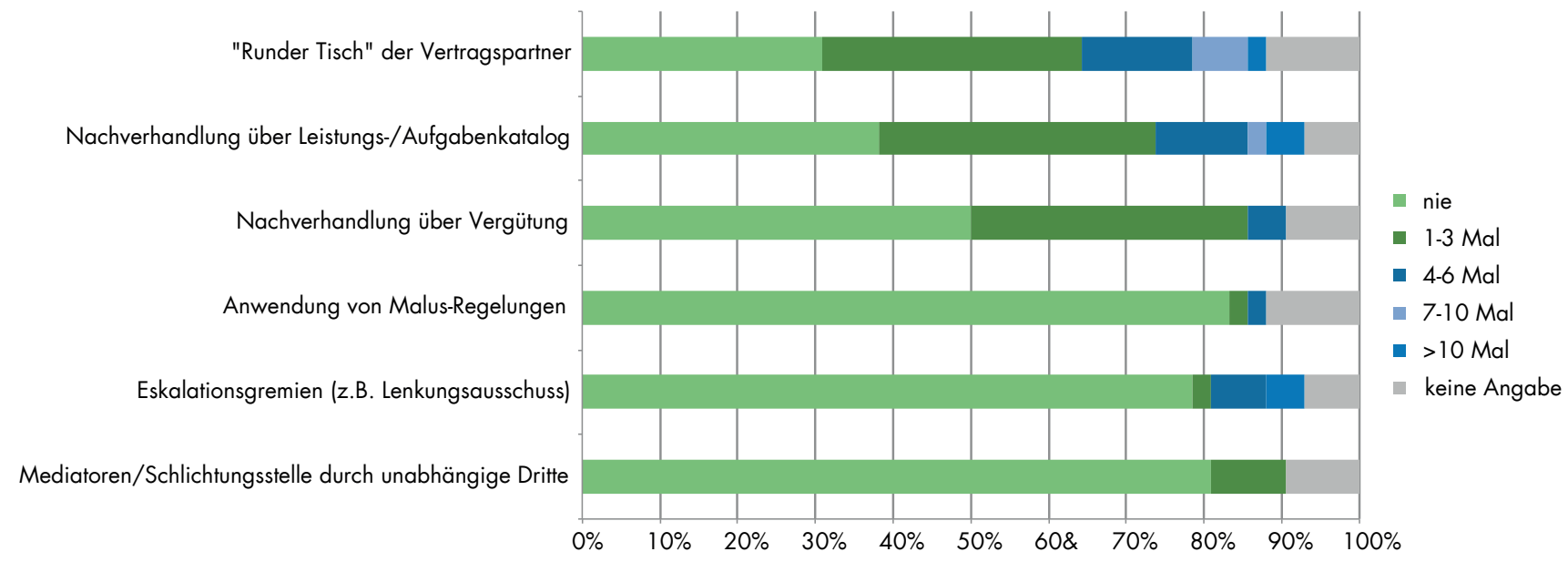

Quelle: Eigene Erhebung, eigene Darstellung, $n=42$.

Eine funktionierende Projektsteuerung ist somit sowohl für die KBV als auch für ÖPP von enormer Relevanz. Die Antworten der im Rahmen dieser Studie befragten Kommunen zeigen, dass sowohl bei der KBV als auch bei ÖPP eher dialogorientierte Steuerungsmechanismen genutz† werden. Bei der KBV werden Gesprächsrunden zwischen den Vertragspartner in Form eines "Runden Tisches" von knapp 57 \% der Kommunen einberufen, wobei bei rund einem Drittel aller Kommunen dies in der Regel ein bis drei Mal im Projektverlauf geschieht. Nachverhandlungen über den Leistungs- und Aufgabenkatalog (55\%) sowie über die zu zahlende Vergütung (41\%) kommen ebenfalls relativ häufig zur Anwendung. Bei den ÖPP-Projekten deckt sich das Bild größtenteils mit dem der konventionellen Projekte. Auch hier kommen dialogorientierte Steuerungsmechanismen am häufigsten zum Einsatz, was dem Anspruch an ÖPP als eine kooperative Partnerschaft Rechnung trägt.
Harte Steuerungsmechanismen, die zu vertraglich geregelten, monetären Folgen für den Auftragnehmer führen, werden bei ÖPP jedoch häufiger als bei der KBV genutzt. Exemplarisch wird dabei auf die Anwendung von Malus-Regelungen rekurriert. Bei diesen kann die Nichteinhaltung von festgelegten qualitativen und quantitativen öffentlichen Zielvorgaben mit finanziellen Abschlägen sanktioniert werden. In ÖPP-Verfahren werden dafür die Leistungen des privaten Partners umfänglich vertraglich geregelt und kontinuierlich überwacht. Beides müsste grundsätzlich bei allen Infrastrukturprojekten geschehen. Doch scheint dies aus zwei Gründen bei der KBV häufig nicht der Fall zu sein: Zum einen stehen hier bei Infrastrukturprojekten häufig die Baukosten im Vordergrund und zum andern ist die Zurechenbarkeit von Leistungsabweichungen oft schwierig. Zum ersten Punkt wurde bereits gezeigt, dass in vielen Kommunen 
die Betriebskosten und -qualität weniger stark präsent sind als die Baukosten. ${ }^{98}$ Die eindeutige Zurechenbarkeit von Leistungsabweichungen ist für die Durchsetzung von harten Steverungsmechanismen, wie Malus-Regelungen, neben der Festlegung von quantitativenund qualitativen Leistungsstandards und deren Überwachung zwingend notwendig. In den Vorbetrachtungen von KBV und ÖPP sollten neben den Bau- grundsätzlich auch die Betriebskosten berücksichtigt werden. In Bau und Betrieb einer Infrastruktureinrichtung sind bei beiden Beschaffungsalternativen vielfältige private Unternehmen eingebunden. Anders als bei ÖPP gibt es bei KBV-Projekten in der Regel aber kein privates Unternehmen, das die Durchführungsverantwortung für die Infrastruktureinrichtung in einer Phase oder gar die für den gesamten Lebenszyklus besitzt: Planung, Bau und Betrieb finden dadurch nicht aus einer Hand statt, sondern jede Phase liegt in der Verantwortung von vielen verschiedenen Unternehmen. Dadurch treten vielfältige Schnittstellenprobleme auf, welche die Überwachung und die eindeutige Zuweisung von quantitativen und qualitativen Leistungsabweichungen bei der KBV erschweren. Im Gegensatz dazu ist bei ÖPP-Projekten ein privater Partner in der Gesamtverantwortung zumeist auch für Betriebsleistungen verantwortlich. Entstehende quantitative und qualitative Abweichungen von bestehenden Leistungsstandards lassen sich diesem im gesamten Lebenszyklus zumeist eindeutig zuweisen. In der Anwendung sollten sich harte Steverungsmechanismen folglich bei ÖPP besser umsetzen lassen als bei der KBV. 


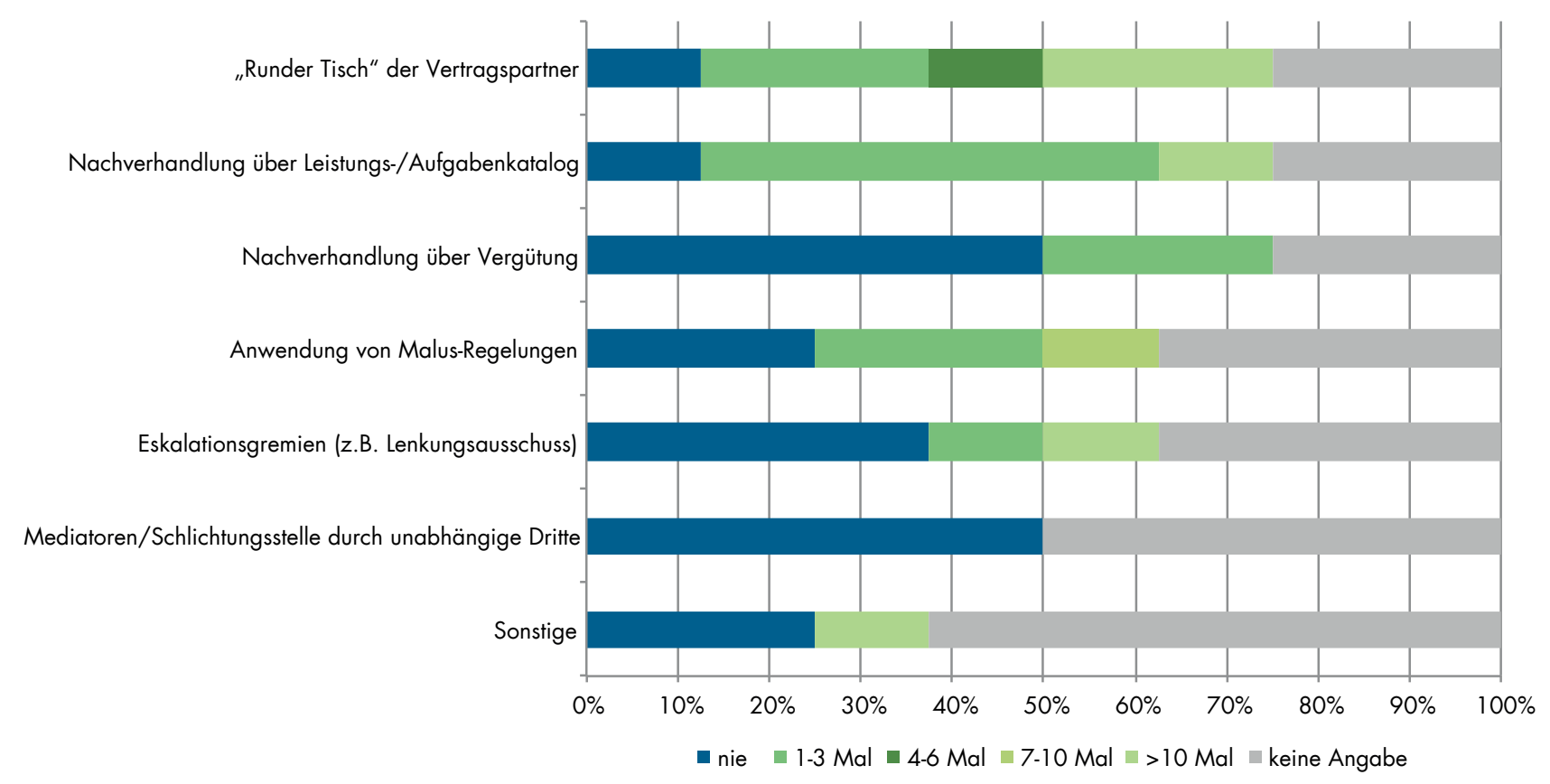

Quelle: Eigene Erhebung, eigene Darstellung, $n=8$.

Die Ergebnisse der Studie bestätigen dies insofern, da von den antwortenden Kommunen im Vergleich $4 \%$ bei KBV- und über $37 \%$ bei ÖPP-Projekten Malus-Regelungen bereits angewendet haben. Die relativ häufigere Nichtanwendung dieses Steuerungsinstruments durch die antwortenden Kommunen bei KBV (80\%) im Gegensatz zu ÖPP (25\%) muss aber nicht zwangsläufig an Problemen der Zurechenbarkeit oder einer fehlenden Implementierung des Instruments liegen. Ebenso kann eine zielgenaue Leistungserbringung vorliegen. Umgekehrt gilt allerdings ebenso, dass eine häufigere Anwendung von MalusRegelungen nicht Ausdruck von schlechter Leistungserfüllung ist. Vielmehr zeigt sich darin, dass eine qualitativ und quantitativ messbare Betriebssteverung mit kontinuierlicher Überwachung besteht.
Mit Blick auf die Projektsteuerung spielen aber nicht nur Steuerungsinstrumente eine Rolle, die vornehmlich mit Beginn der Bau- und Betriebsphase bedeutsam werden. Ebenso wichtig ist eine durchgehende Begleitung des Projektes durch eine Projektgruppe, die aus den verschiedenen, in das Projekt involvierten Stakeholdern zusammengesetzt ist. Die Arbeit dieser Gruppe beginnt oft bereits im Frühstadium des Entscheidungsprozesses mit der Ermittlung des tatsächlichen Bedarfs und begleitet das Projekt durchgehend bis zum Ende der Vertragslaufzeit. In rund der Hälfte der Kommunen (55\%) wird bei der konventionellen Beschaffung eine solche projektbezogene Arbeitsgruppe gebildet. Ihre Aufgaben sind in der Abbildung 22 dargestellt. Am Wichtigsten sind hierbei die Erarbeitung 
von Zielstellungen, die Bedarfsermittlung sowie die Erstellung des Raumkonzepts. Die Arbeit einer Projektarbeitsgruppe ist folglich vor allem in einem frühen Stadium der Planungsphase bedeutsam.

\section{Abbildung 22: Aufgaben der Projektgruppe bei konventionellen Projekten.}

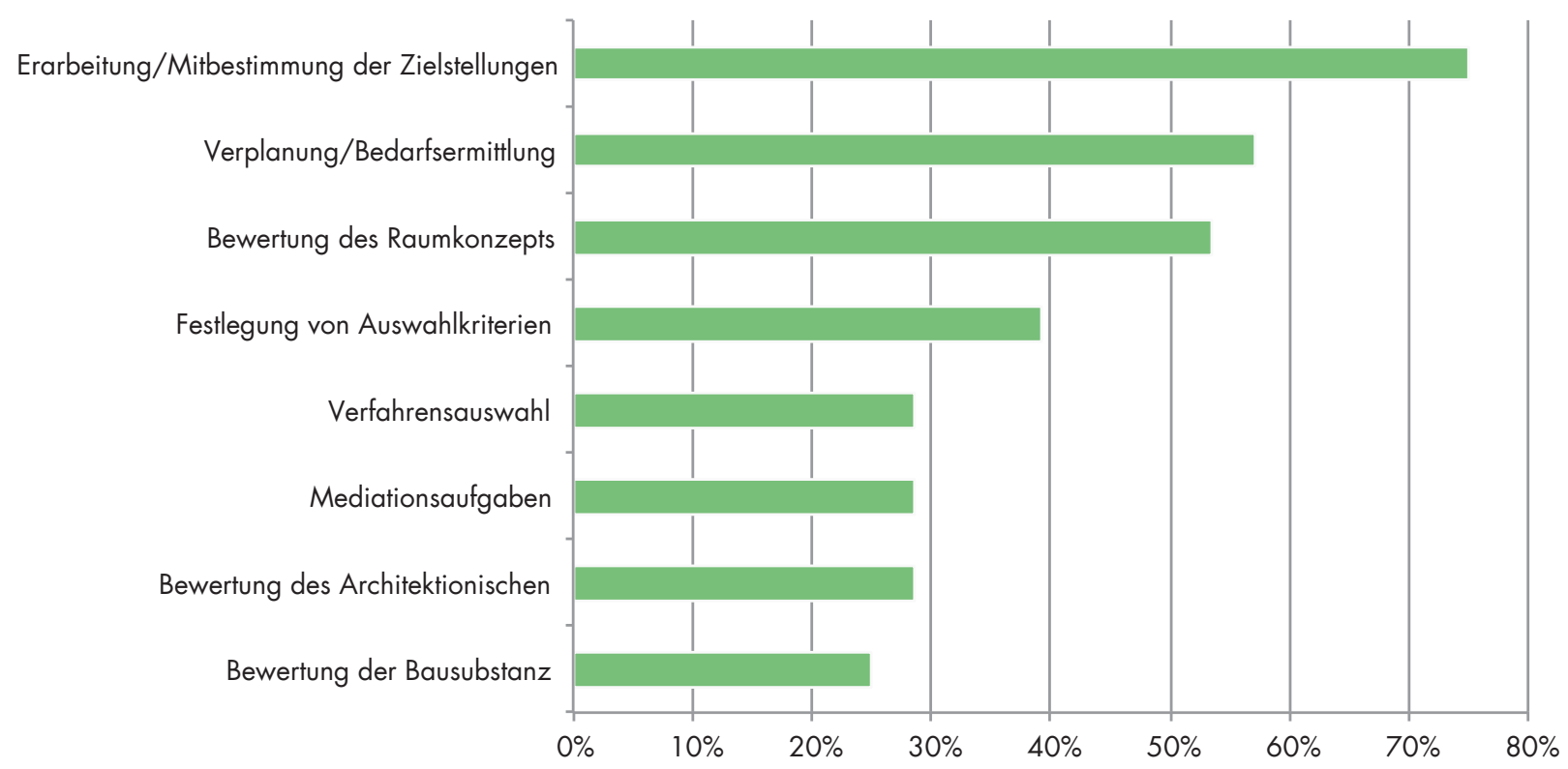

Quelle: Eigene Erhebung, eigene Darstellung, $\mathrm{n}=28$. 


\section{Haushaltstechnische Behandlung}

Die im Zusammenhang mit ÖPP intensiv geführte Transparenzdebatte basiert vor allem auf dem Vorwurf, dass ÖPP-Projekte dazu genutzt werden, Schulden in öffentlichen Haushalten zu verschleiern und Kosten möglichst intransparent darzustellen. ${ }^{99}$ Durch die Umstellung der kommunalen Haushalte auf die doppische Buchführung wird diesen Problemen jedoch größtenteils die Grundlage entzogen. Während bei der Kameralistik nur zahlungswirksame Haushaltsvorgänge verbucht werden, bildet die Doppik durch die Erweiterung um die Ergebnisrechnung den gesamten Ressourcenverbrauch innerhalb einer Periode $a b$ und stellt im Rahmen der Bilanz auch alle noch offenen Zahlungsverpflichtungen dar.

Die Zahlungsströme von KBV und ÖPP unterscheiden sich aus der Sicht der Kommune auf den ersten Blick sehr. Auf den zweiten Blick sind die Unterschiede aber geringer als es zunächst scheint, wobei sich selbstverständlich technische Abweichungen hinsichtlich der angesprochenen Konten und Darstellungsweisen ergeben. ${ }^{100}$ Insgesamt sind die Gemeinsamkeiten und Unterschiede von der gewählten Finanzierungsform abhängig.

Bei Projekten, die als KBV durchgeführt werden, sind die gesamten Zahlungen für die Planungs- und Bauleistungen bis zu deren Fertigstellung zu entrichten (Zahlung bei Übergabe bzw. Abschläge nach Baufortschritt). Die zu erbringenden Investitionsauszahlungen sind in voller Höhe in den kameralen Haushalt bzw. die doppische Finanzrechnung einzustellen. Erfolgt die Finanzierung durch Kredite, sind auch die Einnahmen aus der Kreditaufnahme als zahlungswirksamer Haushaltsvorgang sofort in voller Höhe im Haushalt einzustellen. Bei Kreditfinanzierung werden in den Folgejahren Auszahlungen für den Schuldendienst (Zinsen und Tilgung bzw. Rücklagenbildung bei Endfälligkeit) geleistet, wobei nicht notwendigerweise eine Kongruenz zwischen der Nutzungsdauer und der Kreditlaufzeit herrscht. Selbstfinanzierte Anteile tauchen hingegen in den Folgejahren sowohl in der kameralen Haushaltsrechnung als auch in der doppischen Finanzrechnung nicht mehr auf. ${ }^{101}$

Bei einer ÖPP-Realisierung ist charakteristisch, dass sämtliche Zahlungen periodisch im geplanten Lebenszyklus der Infrastruktureinrichtung (zumeist ÖPP-Vertragsdaver) anfallen. Vor der Fertigstellung findet zunächst kein zahlungswirksamer Haushaltsvorgang statt. Umfasst das ÖPP-Projekt die Finanzierung mittels Forfaitierung, so entfällt eine einmalige Anfangsauszahlung, die auch nicht durch eine korrespondierende einmalige Einzahlung aus der ggf. erforderlichen Kreditaufnahme begleitet wird. Dies bedeutet aber keinesfalls eine Verschleierung der Belastungen. Die jährlich durch die öffentliche Hand zu erbringenden Zahlungen umfassen sowohl laufende Betriebsleistungen als auch Anteile für die Planungs- und Bauleistungen. Damit korrespondieren die Zahlungsströme mit der tatsächlichen Nutzungsdauer und es findet eine Angleichung von Zahlungsmittelund Ressourcenverbrauch statt. Insbesondere werden selbstfinanzierte Anteile an den Investitionsauszahlungen während der Laufzeit direkt sichtbar. In der doppischen Haushaltsführung werden somit Ergebnis- und Finanzrechnung synchronisiert. Die noch offenen Verpflichtungen aus ÖPP-Verträgen sind durch bilanzielle Passivposten erkennbar.

\footnotetext{
99 Vgl. Zur Problematik ÖPP und öffentliche Verschuldung Mühlenkamp (2012). Zum Vorwurf der verdeckten öffentlichen Verschuldung mit Hilfe von ÖPP u.a. Die Linke (2012) und Schumann (2013).

Zur Darstellung von ÖPP-Projekten im kameralen und doppischen Haushalt erstellt das ÖPP-Kompetenzzentrum Sachsen derzeit eine eigenständige Studie, die Ende des Jahres 2013 erscheinen wird.

Die Stellung in der Ergebnisrechnung mit Aktivierung der Positionen und Abschreibung über die Laufzeit werden an dieser Stelle nicht behandelt.
} 
Die skizzierten Unterschiede können jedoch relativiert werden. Die Zahlungsströme von ÖPP-Projekten mit Forfaitierung entsprechen prinzipiell dann denen einer KBV-Umsetzung, wenn in der KBV-Variante vollständig kreditfinanziert und hinsichtlich der kommunalen Kredite ein Gleichlauf der Nutzungsdauer von Infrastruktur und Kreditlaufzeit hergestellt wird. Der Unterschied liegt in diesem Fall lediglich in den Spitzen, die beim Kommunalkredit auf der Ausgabenseite (Investitionen) und Einnahmeseite (Einnahme aus Krediten) bestehen, sich aber vollständig saldieren. Insofern lässt sich eine "Neutralisierung" der Zahlungsstrukturen zwischen ÖPP und KBV herstellen, wenn eine entsprechende nutzungsäquivalente Finanzierung gewählt wird.

Eine Finanzierung mittels Forfaitierung ist jedoch nicht notwendige Voraussetzung für die lebenszyklusorientierte Umsetzung. Ebenso ist eine Trennung der Betriebs- von den Investitionsanteilen denkbar. Während die Betriebsleistungen parallel zur Nutzungsdauer haushaltswirksam werden, kann der Investitionsanteil wie bei einer KBV haushaltstechnisch dargestellt werden.

Insgesamt ist die haushaltstechnische Belastung von Infrastruktureinrichtungen im Allgemeinen und von ÖPP-Projekten im Besonderen in der Doppik klarer ersichtlich und kann besser nachvollzogen werden als im kameralen System, da nun auch Positionen für die erst in Zukunft anfallenden Zahlungen eines Investitions- oder ÖPP-Projekts gebildet werden müssen. Zudem sind die korrespondierenden Aktiv- und Passivposten in der kommunalen Bilanz ausgewiesen. Im kameralen Haushalt war dies bisher nicht der Fall, was in der Vergangenheit dazu führte, dass zukünftige Zahlungsverpflichtungen aus ÖPP-Projekten nicht gleichberechtigt neben
Kreditmarktschulden auszuweisen waren. Diese Verbesserungen sind auf einen allgemeinen Transparenzgewinn durch die Doppik zurückzuführen. Vermeintliche Transparenzdefizite vom ÖPP bei kameraler Haushaltsführung sind nicht der Beschaffungsvariante anzulasten. Im rein zahlungsorientierten System der Kameralistik hat ein ÖPP-Projekt mit Forfaitierung sogar deutliche Transparenzvorteile gegenüber der KBV, da die Haushaltsbelastungen auf die tatsächliche Nutzungsdaver projiziert werden.

Vondenteilnehmenden Kommunennutzen rund zwei Drittel (65 \%) bereits die kaufmännische Buchhaltung, während die Kameralistik nur noch von einem Drittel verwendet wird. ${ }^{102}$ In allen Bundesländern sind die Regelungen zur Einführung der Doppik auf der kommunalen Ebene gesetzlich verankert, ${ }^{103}$ jedoch sind die Umstellungsfristen je nach Bundesland unterschiedlich festgesetzt. So sind die Kommunen in der Mehrzahl der Bundesländer bereits verpflichtet, doppisch zu buchen. Bis 2011 wurde in acht Ländern das kommunale Rechnungswesen umgestellt, 2013 folgte Sachsen. Andere Länder haben ihren Städten und Gemeinden noch längere Umstellungsphasen zugebilligt. So müssen die Kommunen in Baden-Württemberg erst ab dem Jahr 2020 ihre Haushalte endgültig auf das neue Buchungswesen umstellen. In Thüringen (seit 2009), Bayern und Schleswig-Holstein (beide seit 2007) haben die Landesgesetzgeber ihren Kommunen die Wahlmöglichkeit zwischen der Nutzung der Doppik oder der Kameralistik eingeräumt. ${ }^{104}$

Die Kommunen Berlin, Bremen und Hamburg zählen aufgrund ihres Status als Stadtstaaten zur Länderebene und werden hier nicht mit betrachtet. Hamburg und Bremen haben die Doppik bereits eingeführt. Berlin sieht lediglich die Umstellung auf die erweiterte Kameralistik vor, die Doppik wird vorerst nicht eingeführt. 


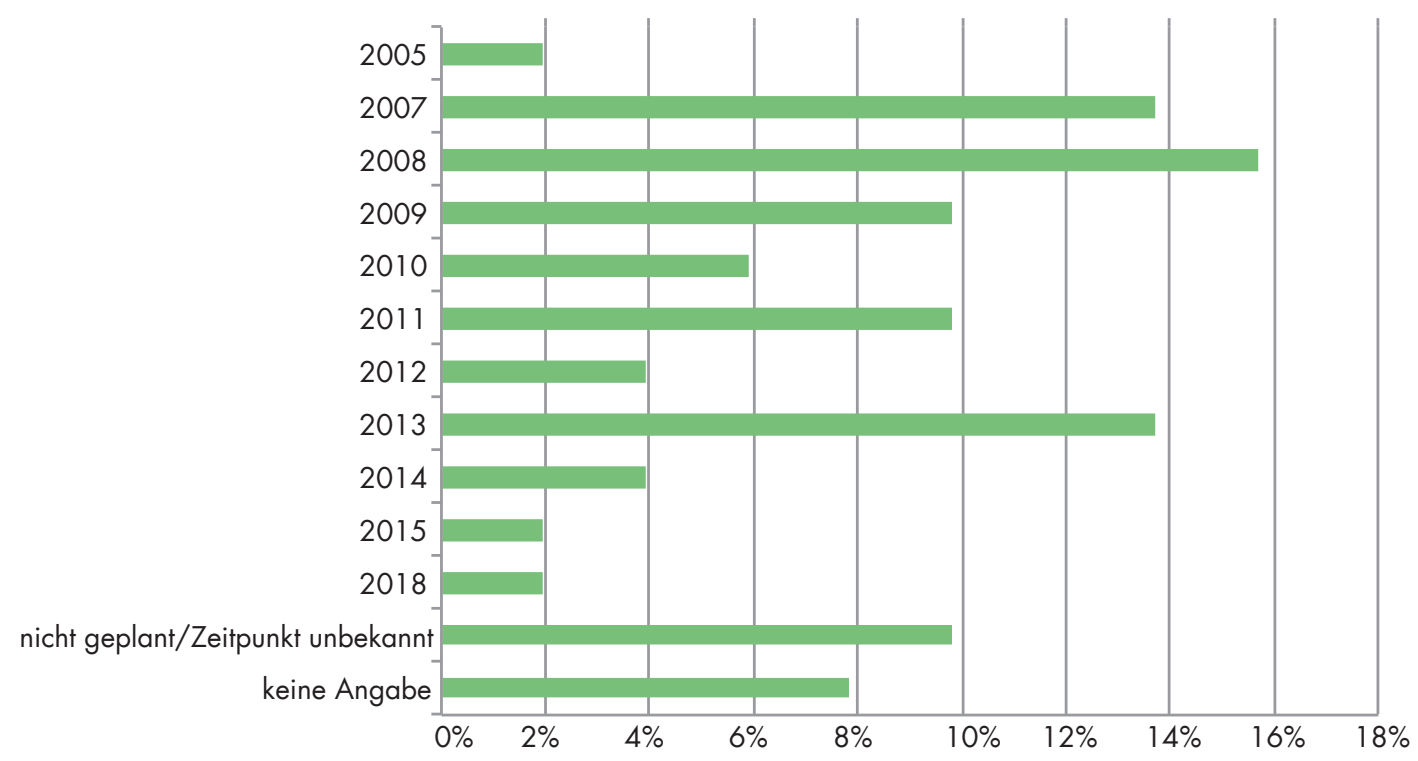

Quelle: Eigene Erhebung, eigene Darstellung, $\mathrm{n}=51$.

Abbildung 23 gibt eine Übersicht, wann die befragten Kommunen ihre Buchführung umstellten bzw. wann sie diese umstellen werden. Demnach hat die Mehrzahl der 51 Kommunen bereits die Doppik eingeführt, rund $22 \%$ werden die Umstellung in den Jahren von 2013 bis 2020 in Angriff nehmen. Bei 10\% der Kommunen ist der Zeitpunkt der Umstellung noch unbekannt bzw. es war zur Zeit der Befragung noch keine Umstellung in Planung. Somit gilt für die Mehrzahl der Kommunen, dass die Vorwürfe gegen die Intransparenz der haushaltstechnischen Darstellung der ÖPP-Projekte und den Rückgriff auf diese alternative Beschaffungsvariante aus reinen Finanzierungsaspekten für die kommunale Ebene nicht (mehr) aufrecht gehalten werden können. Zudem wird durch die Darstellung des gesamten Ressourcenverbrauchs in der Doppik ebenso die Transparenz der KBV erhöht. Werden Folgekosten wie Sanierungs- und
Instandhaltungsarbeiten aufgeschoben, wird der Ressourcenverzehr im doppischen Rechnungswesen dennoch in Höhe der Abschreibungen sichtbar. In der Kameralistik hingegen wird der Verschleiß der kommunalen Infrastruktur nicht ersichtlich, da nur zahlungswirksame Ströme berücksichtigt werden. 


\section{Abschließender Vergleich zwischen konventioneller und ÖPP-Realisierung}

Mit Hilfe empirischer Daten wurde in der vorliegenden Studie ein erster Versuch unternommen, die von den Rechnungshöfen in ihrem Erfahrungsbericht und die auch anderweitig geäußerte Kritik am ÖPP-Beschaffungsprozess in gegenüberstellender Betrachtung zu untersuchen. Hierbei steht der Vergleich der Beschaffungsrealität bei KBV und ÖPP im Vordergrund. Dies insbesondere, da die Anforderungen, die an die alternative Beschaffungsvariante ÖPP gestellt werden, grundsätzlich ebenso für die zumeist eingesetzte KBV ihre Gültigkeit besitzen. In diesem Kontext ist darauf hinzuweisen, dass das Ziel der Studie nicht darin liegt, ÖPP als den "Königsweg” der öffentlichen Beschaffung darzustellen, sondern einen kritischen Blick auf die Beschaffungsvariante als auch auf die an ihr geäußerte Kritik zu werfen. Viele der aufgedeckten Probleme und Unzulänglichkeiten sind nicht der Beschaffungsvariante ÖPP-immanent, sondern treten ebenso bei der KBV auf. Insgesamt wurde für den öffentlichen Beschaffungsprozesses in Gänze ein Verbesserungsbedarf aufgezeigt. Eine konsequente Implementierung von ÖPP immanenten Elementen, wie beispielsweise eine lebenszyklusorientierte WU und die Durchführung von Risikoanalysen, könnten dabei helfen, diesen abzubauen. Die geäußerte Kritik der Rechnungshöfe an OPP, die Medienberichterstattung und die existierenden gesellschaftlichen Vorbehalte vermitteln hingegen einen anderen Eindruck.

Dies führt zu der Vermutung, dass letztlich die öffentlichen Auftraggeber, die eine langfristige Partnerschaft mit einem privaten Unternehmer eingehen und somit ÖPP für ihre Infrastruktureinrichtungen nutzen, mehrheitlich eher unzufrieden sein müssten.
Dieses Bild bestätigt sich bei Vorträgen der Kommunen mit ÖPP-Erfahrung, die beispielsweise bei kommunalen Konferenzen, Projektgesprächen und Fachtagungen präsentiert werden, nicht. Die öffentlichen Partner der dort vorgestellten Projekte urteilen durchaus differenziert zum Thema ÖPP, kommen aber insgesamt zu einer positiven Gesamtbewertung. Zumeist wird die sehr gute Zusammenarbeit und partnerschaftliche Lösungsfindung hervorgehoben. Gleichzeitig wird dabei aber ebenso betont, dass viele Projekte vielfältige Herausforderungen überstehen mussten. Die Änderung politischer Mehrheitsverhältnisse und ein aufkommender gesellschaftlicher Widerstand spielen dabei durchaus eine Rolle, ebenso wie Kostensteigerungen während der ÖPP-Wi rtschaftlichkeitsuntersuchung, wie sie auch von den Rechnungshöfen benannt wurden. Diese Erhöhungen der Projektkosten bis zur abschließenden WU sind jedoch zumeist nicht den privaten Partnern anzulasten. Vielmehr liegen diese an möglicherweise zu niedrigen Kostenschätzungen in der vorläufigen WU, an einer unzureichenden Bestands- und Bedarfsanalyse und unvollständigen Einschätzung der benötigten Leistungen sowie an zusätzlichen und erst nachträglich eingebrachten Nutzeransprüchen. Diesbezüglich wird von öffentlicher Seite betont, dass ÖPP durch die langfristige finanzielle Sicherheit ein Instrument zur Disziplinierung darstellt. ${ }^{105}$ Der phasenübergreifende Planungsansatz von ÖPP kann in Verbindung mit der intensiven Einbeziehung des Know-hows und der Projekterfahrung des privaten Partners einerseits unbedachte Bedarfe und Nutzeransprüche noch während der Planung offenlegen. Zusätzliche Investitionsund Folgekosten für Leistungen, die bei der 
Erstellung des PSC für die KBV nicht bedacht wurden, können so transparent ausgewiesen und dessen Einbeziehung abgewogen werden. Andererseits kann der private Partner bei ÖPP besser als bei der KBV eigene (innovative) Planungs- und Lösungsansätze entwickeln und bereits in die Planungsphase einbringen. Vor dem Hintergrund der langfristigen Bindung des privaten Partners und der damit verbundenen Verantwortungs- und Risikoteilung kann dies dazu führen, dass wirtschaftlichere und bedarfsgerechtere Lösungen als bei der KBV gefunden werden.

Aufgrund des phasenübergreifenden Planungsansatzes ist es bei ÖPP zwingend notwendig, die Nutzer frühzeitig - bereits in der Planungsphase - einzubeziehen, um alle Anforderungen vor Zuschlagserteilung auch entsprechend berücksichtigen zu können. Dementsprechend spielen Anhörungs-, Vorschlags-, Mitentscheidungsund Gewichtungsmöglichkeiten sowie Bedarfsabfragen eine entscheidende Rolle und wurden bei allen befragten Kommunen während der Projektplanung eingesetzt. Die vorläufige WU als erste grobe Entscheidungsgrundlage wird jedoch häufig ohne fundierte Partizipationsmöglichkeiten der Nutzer erstellt. Dabei bilden, wie bei der KBV üblich, standardisierte Qualitäten und Kostensätze, verwaltungsinterne Erfahrungsund Schätzwerte sowie externes Know-how die Grundlage. Dabei ist zu beachten, dass die Implementierung des ÖPP-Ansatzes vielfach ein Novum darstellt. Das Verständnis, dass alle Projektanforderungen zu Beginn formuliert werden müssen, ist häufig nicht vorhanden und muss erstgeschaffen werden. ${ }^{106}$ Diesliegtdaran, dass bei vielen konventionellen Projekten, insbesondere was Nutzeranforderungen wie die Ausstattungsmerkmale von Räumen betrifft, diese entweder jederzeit bzw. bis zum Ende der Bauphase noch eingebracht werden konnten oder dass den Nutzern gar kein Mitspracherecht eingeräumt wurde. Dadurch entstehen bei ÖPP-Projekten Abweichungen zwischen der vorläufigen und der abschließenden WU, die einer bedarfsgerechteren Planung geschuldet sind, aber im Vergleich zur KBV zu einer größeren Kostensicherheitbeim Projektabschluss führen. Die zusätzlichen Leistungen und Kosten fallen ebenso bei der KBV an, weshalb auch vor diesem Hintergrund die Forderung, den PSC kontinuierlich fortzuschreiben, gerechtfertigt ist. Dabei ist allerdings zu beachten, dass innovative Planungs- und Lösungsansätze, die erst aufgrund des phasenübergreifenden Planungsansatzes von ÖPP gefunden wurden, auch nur diesem angerechnet werden können. ${ }^{107}$ Nachträge lassen sich bei kaum einem Projekt vermeiden, so auch nicht bei ÖPP. Doch ermöglicht dessen Ansatz im Gegensatz zur konventionellen Realisierung, diese sofort einzupreisen und die finanziellen Auswirkungen für die Gesamtnutzungsdaver aufzuzeigen. Wie Abbildung 24 zeigt, ist eine Abweichung zwischen den Annahmen der abschließenden WU und den tatsächlich eingetretenen Ergebnissen eine Ausnahmeerscheinung.

\footnotetext{
$106 \quad$ Kritisch anzumerken ist dazu allerdings, dass nicht alle im Lebenszyklus der Infrastruktureinrichtung notwendigen Anpassungen an die Nutzung von Anfang an bedacht werden können. Insofern bestehen diesbezüglich aber auch gewisse Grenzen. Auf die damit korrespondierende Problematik von unvollständigen Verträgen bei langfristigen vertraglichen Bindungen haben u. a. Budäus/Grüb 2008 hingewiesen. 


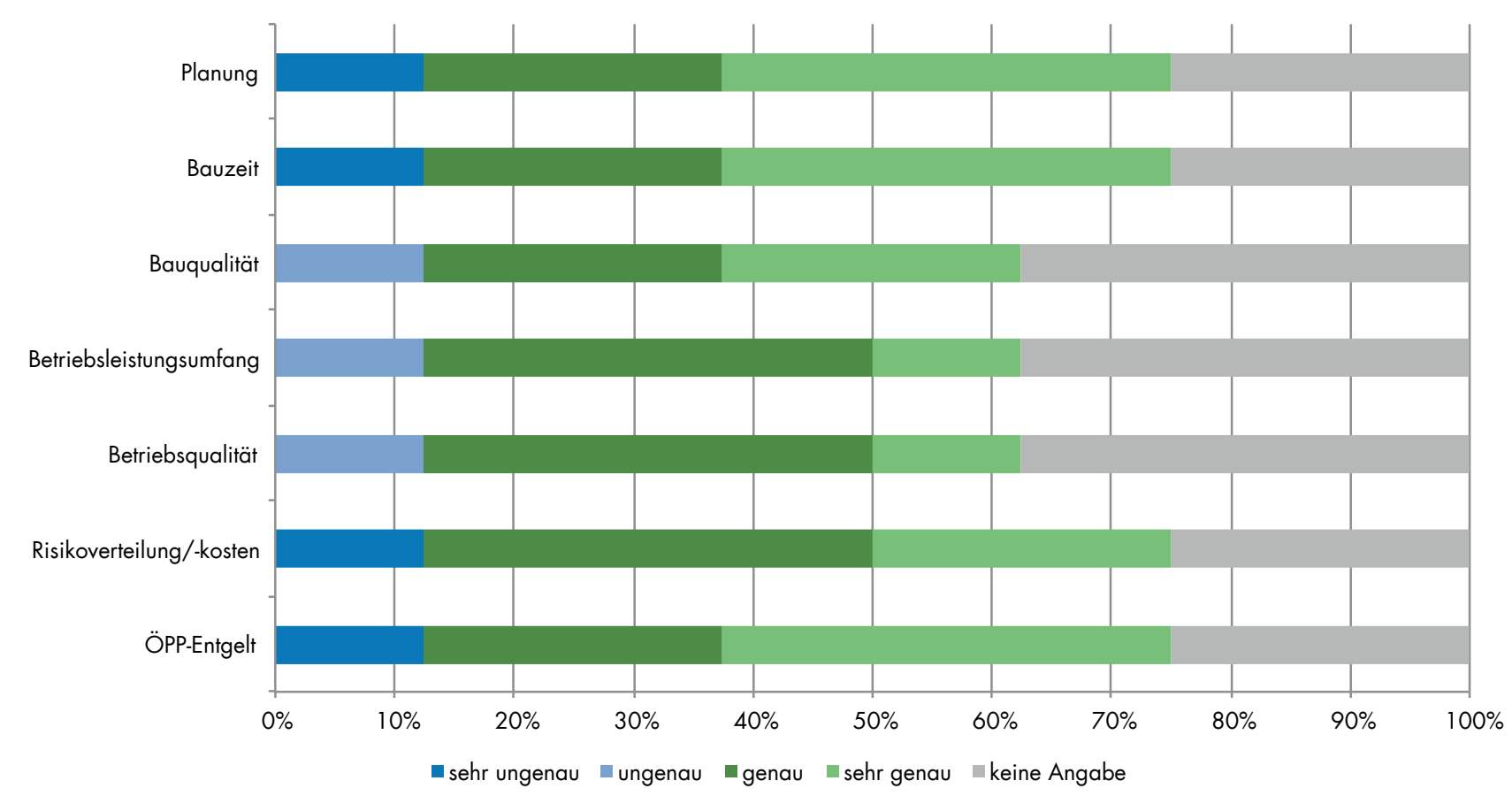

Quelle: Eigene Erhebung, eigene Darstellung, $n=8$.

Aufgrund des lebenszyklusorientierten Planungsansatzes, des Wirtschaftlichkeitsvergleichs und der die gesamte Projektlaufzeit umfassenden Einbeziehung eines privaten Partners ist es somit schwieriger als bei der KBV, rein politisch intendierte Projekte bis zum Projektstart der faktischen Berechnungsbasis zu entziehen, folglich "nach außen" zu optimieren. In vielen Fällen können durch eine klare Bestimmung der notwendigen Zielgrößen und Leistungen i. V. m. der frühzeitigen Einbeziehung der Nutzer Nachträge und Planungsänderungen aufgrund weiterer Leistungsanforderungen abgewehrt werden. Das Ergebnis der Umfrage verdeutlicht allerdings, dass die Nutzer noch stärker in die Projektierung einbezogen werden können. Insbesondere Mitentscheidungselemente wie Gewichtungen sind noch ausbaufähig.
$80 \%$ der Kommunen, die bereits ÖPP-Erfahrungen besitzen, wollen wieder auf dieses Beschaffungsinstrument zurückgreifen. Nur eine der befragten Kommunen wollte sich zu dieser Frage nicht äußern.

Insgesamt zeigen die Ergebnisse dieser Studie, dass die lebenszyklusorientiere Planung und Bewirtschaftung von Infrastruktur in der kommunalen Beschaffungsrealität noch zu wenig berücksichtigt wird. Da entweder gar keine WU oder diese mit einer zu starken Fokussierung auf die Anschaffungskosten durchgeführt wird, werden die Gesamtkosten der Infrastruktur vielfach nur unzureichend analysiert. Die KBV lässt dabei substanzielle Effizienzpotentiale ungenutzt. Aufgrund der Trennung von Planung, Bau und Betrieb entstehen Schnittstellenkosten, während 
innovative Lösungsansätze vielfach nicht oder zu spät entwickelt werden können. Gerade vor dem Hintergrund der knappen Ressourcen kann der ÖPP-Ansatz als alternative Beschaffungsvariante oder als Treiber für die Modernisierung der konventionellen Beschaffung für die öffentliche Hand ein hilfreiches Instrument darstellen. 
58 


\section{Literaturverzeichnis}

Albrecht, Romy/Grüttner, André/Lenk, Thomas/Lück, Oliver/Rottmann, Oliver (2012): Optionen moderner Bürgerbeteiligung bei Infrastrukturprojekten. Ableitungen für eine verbesserte Beteiligung auf Basis von Erfahrungen und Einstellungen von Bürgern, Kommunen und Unternehmen. Studie des Kompetenzzentrums Öffentliche Wirtschaft, Infrastruktur und Daseinsvorsorge e.V. an der Universität Leipzig.

Anton, Stefan/Diemert, Dörte (2012): Stabile Stadtfinanzen - nur mit Bund und Ländern, in: Deutscher Städtetag (Hrsg.), Gemeindefinanzbericht 2012, S. 9-23.

Arbeitsgruppe der ÖPP-Kompetenzzentren (2013): Stellungnahme einer Arbeitsgruppe der ÖPP-Kompetenzzentren der Länder Mecklenburg-Vorpommern, Niedersachsen, Nordrhein-Westfalen, Rheinland-Pfalz, Sachsen, SchleswigHolstein und Thüringen zum Bericht der Rechnungshöfe des Bundes und der Länder "Gemeinsamer Erfahrungsbericht zur Wirtschaftlichkeit von ÖPP, online verfügbar unter: http://www.uni-leipzig.de/fiwi/Kompetenzzentrum/ $\mathrm{pdf} /$ Stellungnahme\%2OBRH-Bericht.pdf.

Arbeitsgruppe Wirtschaftlichkeitsuntersuchungen bei PPP-Projekten (2006): Leitfaden Wirtschaftlichkeitsuntersuchungen bei PPP-Projekten, online verfügbar unter: http://www.bmvbs.de/cae/servlet/contentblob/3 1824/publicationFile/631/leiffaden-wirtschaftlichkeits-untersuchung-bei-ppp-projekten-september-2006.pdf.

Attac (o.J.): PPP stoppen - 12 Jahre sind genug! online abrufbar unter: http://www.attac.de/startseite/detailansicht/datum/2012/01/25/ppp-stoppen-12-jahre-sind-genug.

Baukosteninformationszentrum Deutscher Architektenkammern (BKI) (2012): BKI Objektdaten. NK4. Nutzungskosten.

Beckers, Thorsten/Klatt, Jan Peter (2009): Kosteneffizienz von Public-Private-Partnerships. Erwartungen und empirische Erkenntnisse, in: Wirtschaftsdienst, 3/2009, S. 176-183.

Beckers, Thorsten/Wagemann, Felix/Klatt, Jan Peter (2013): Wirtschaftlichkeitsuntersuchungen bei PPP-Vorhaben, in: Reichard, Christoph/Schröter, Eckhard (Hrsg.): Zur Organisation öffentlicher Aufgaben: Effizienz, Effektivität und Legitimität, Festschriff für Manfred Röber, S. 148-183.

Beratergruppe - PPP im öffentlichen Hochbau (2003): PPP im öffentlichen Hochbau. Band I: Leiffaden; online verfügbar unter: http://www.bmvbs.de/cae/servlet/contentblob/31808/publicationFile/10547/gutachten-ppp-imoeffentlichen-hochbau-band-1.pdf.

Bodner, Herbert (2011): Jeder achte kommunale Auftraggeber empfindet heute Widerstände in Presse und Öffentlichkeit als ÖPP-Hemmnis, Gastbeitrag vom 3. Juni 201 1, online verfügbar unter: http://www.oepp-plattform.de/ infocenter/opp-kommentar/_/artikel/jeder-achte-kommunale-auftraggeber-empfindet-heute.

Budäus, Dietrich/Eichhorn, Peter (Hrsg.) (1997): Public Private Partnership. Neue Formen öffentlicher Aufgabenerfüllung, 1.Aufl., Schriftenreihe der Gesellschaft für öffentliche Wirtschaft, H. 41, Baden-Baden.

Budäus, Dietrich/Grüb, Birigt (2008): Public Private Partnership (PPP): Zum aktuellen Entwicklungs- und Diskussionsstand, in: Bauer, Hartmut/Büchner, Christiane/Brosius-Gersdorf, Frauke (Hrsg.): Verwaltungskooperation. Public Private Partnerships und Public Public Partnerships, KWI Schriften 1, Magdeburg, S.33-50, online verfügbar unter: http://opus.kobv.de/ubp/volltexte/2009/2758.

Bundesministerium für Verkehr, Bau und Stadtentwicklung (BMVBS) (Hrsg.) (2009): Energieausweis für Gebäude - nach Energieeinsparverordnung (EnEV 2009), online verfügbar unter: http://www.bmvbs.de/cae/servlet/contentblob/24270/publicationFile/656/broschuere-energieausweis-fuer-gebaeude-nach-energieeinsparverordnungenev-2009.pdf.

Deutscher Bundestag (201 1): Transparenz in Public Private Partnerships im Verkehrswesen. BT-Drucks. 17/5258, Berlin. 
Deutscher Städtetag (2012): Stabile Stadtfinanzen -nur mit Bund und Ländern. Schlaglichter aus dem Gemeindefinanzbericht 2012 des Deutschen Städtetages, in: Beiträge des Deutschen Städtetages zur Stadtpolitik, Band 97, Berlin, online verfügbar unter: http://www.staedtetag.de/imperia/md/content/dst/veroeffentlichungen/gemeindefinanzbericht/gfb2012_broschuere_.pdf.

Deutscher Städtetag (2010): Konjunkturpaket II: Städte haben nachhaltig investiert und schöpfen Mittel aus - vereinfachte Vergabe verlängern, Pressemitteilung des Deutschen Städtetags, 30.12.2010, online verfügbar unter: http:// www.deutscher-städtetag.de/presse/mitteilungen/058101/index.html.

Gatzke, Nicolas (2010): Public Private Partnerships und öffentliche Verschuldung, in: Rürup, Bert/Sesselmeier, Werner (Hrsg.), Sozialökonomische Schriften, Nr. 41, Frankfurt a.M.

Grabow, Busso (2008a): PPP und Mittelstand - Untersuchung von 30 ausgewählten Hochbauprojekten in Deutschland, Deutsches Institut für Urbanistik, Berlin, online verfügbar unter: http://www.bmvbs.de/cae/servlet/contentblob/32222/publicationFile/682/ppp-und-mittelstand-endbericht.pdf.

Grabow, Busso et al. (2008b): PPP - Wirtschaftlichkeit, Qualitäten, Beratung, Partnerschaft. Ergebnisse einer ergänzenden Umfrage zum Projekt „PPP und Mittelstand". Deutsches Institut für Urbanistik, Berlin, online verfügbar unter: http://edoc.difu.de/edoc.php?id=L5I7GWKT.

Grabow, Bussow et al. (2009): PPP-Projekte in Deutschland 2009 - Erfahrungen, Verbreitung, Perspektiven, Deutsches Institut für Urbanistik, Berlin.

Greiling, Dorothea (2009): Public Private Partnership - a driver for efficient public services or just an example of wishful thinking, in: Zeitschrift für öffentliche und gemeinwirtschaftliche Unternehmen, Beiheft 37, S. 108-125.

Hamann, Götz (2009): Die nächste Privatisierungswelle, in: Zeit Online, 08.10.2009, online verfügbar unter: http://www.zeit.de/2009/42/PPP.

Hauptverband der Deutschen Bauindustrie (2013): Thesenpapier des Hauptverbandes der Deutschen Bauindustrie Anlässlich des Gesprächs mit dem AK Bau der Rechnungshöfe von Bund und Ländern, online verfügbar unter: http://www.oepp-plattform.de/media/attachments/130503_Thesenpapier_AK_BAU_LRH_v3_4.pdf.

Hesse, Mario/Lück, Oliver/Redlich, Matthias (2012): Kommunaler Investitionsbedarf und ÖPP in Sachsen. Ergebnisse einer empirischen Studie des ÖPP-Kompetenzzentrums Sachsen 2011/2012, online verfügbar unter: http:// www.uni-leipzig.de/fiwi/Kompetenzzentrum/pdf/121012 Studie PPP Umfrage Endfassung.pdf.

Hofreiter, Anton (2012): Die Finanzierungsfalle, taz.de, 12.02.2012, online verfügbar unter: http://www.taz. de/!87535.

IfD Allensbach (201 1): Die Zufriedenheit mit ÖPP-Projekten im Schulbereich aus Sicht von Auftraggebern, Schulleitern und Elternvertretern. Ergebnisse einer repräsentativen Umfrage, Institut für Demoskopie Allensbach, online verfügbar unter: http://www.ifd-allensbach.de/uploads/tx_studies/7587.OEPP-Projekte.pdf.

KfW Bankengruppe (Hrsg.) (2012): KfW-Kommunalpanel 2012, Berlin, online verfügbar unter: http://www.difu. de/sites/difu.de/files/kfw-kommunalpanel_2011_If.pdf.

Kiefer, Dieter (2007): Public Private Partnership (PPP) in Deutschland. Vortrag beim IV. EURORAI-Kongress, 21. - 23.10.2007 in Crans-Montana, online abrufbar unter: http://www.eurorai.org/PDF/pdf\%20congress\%20 Crans-Montana/Praesentation-Kiefer.pdf.

Landesrechnungshof Sachsen-Anhalt (2012): Haushalts und Wirtschaftsführung im Haushaltsjahr 2011 . Teil 1 Denkschrift und Bemerkungen, online verfügbar unter: http://www.Irh.sachsen-anhalt.de/fileadmin/user_upload/ Berichte/12a.pdf 
Lenk, Thomas/Röber, Manfred/Kuntze, Martina/Redlich, Matthias/Rottmann, Oliver (201 1): Public-Private Partnership-An Appropriate Institutional Arrangement for Public Services? Beiheft 41/2011 der Zeitschrift für öffentliche und gemeinwirtschaftliche Unternehmen (ZögU).

Lenk, Thomas/Rottmann, Oliver/Albrecht, Romy/Grüttner André (2012): Auswirkungen der Energiewende für die Stadtwerke, Studie des Kompetenzzentrums Öffentliche Wirtschaft, Infrastruktur und Daseinsvorsorge e.V. an der Universität Leipzig in Kooperation mit der HypoVereinsbank.

Lenk, Thomas/Hesse, Mario (201 1): Gemeindefinanzbericht Sachsen 2010/201 1, in: Sachsenlandkurier, 5/2011, Dresden.

Lenk, Thomas (2009): Gemeindefinanzbericht Sachsen 2008/2009, in: Sachsenlandkurier, 5/2009, Dresden.

Maier, Wolfgang (2000): Die Nagelprobe brachte den Beweis, in: Bundesgemeinschaft der Ingenieurkammern Deutschlands (Hrsg.), Deutsches IngenieurBlatt, Heft 10, Oktober 2000, S. 34-36.

Mühlenkamp, Holger (2006): Public Private Partnership aus der Sicht der Transaktionskostenökonomik und der Neuen Politischen Ökonomie, in: Budäus, Dietrich (Hrsg.), Kooperationsformen zwischen Staat und Markt. Theoretische Grundlagen und praktische Ausprägungen von Public Private Partnership, Schriftenreihe der Gesellschaft für öffentliche Wirtschaft, Heft 54, Baden-Baden, S. 29-48.

Mühlenkamp, Holger (2010): Ökonomische Analyse von Public Private Partnerships (PPP) -PPP als Instrument zur Steigerung der Effizienz der Wahrnehmung öffentlicher Aufgaben oder als Weg zur Umgehung von Budgetbeschränkungen?, Discussion Paper Nr. 55, Deutsches Forschungsinstitut für öffentliche Verwaltung Speyer.

Mühlenkamp, Holger (2012): Effizienzgewinne und Entlastungen öffentlicher Haushalte durch Public Private Partnership (PPP)?, in: Küpper, Ulrich/Semper, Lothar (Hrsg.): Chancen und Risiken von PPP - Eine Betrachtung aus ökonomischer und juristischer Perspektive, München, S. 63-126.

Napp, Hans-Georg (2011): Die Finanzierung von öffentlich-privaten Partnerschaften (PPP) -Grundlagen und aktuelle Entwicklungen, in: Kommunalwirtschaft, Sonderausgabe 2011 , S. 53-59.

ÖPP Deutschland AG (2010): Auswirkungen der Finanzmarktkrise auf Öffentlich-Private Partnerschaften, insbesondere im Hochbau, in: ÖPP-Schriftenreihe, Band 1.

ÖPP Deutschland AG (2012): Öffentlich-Private Partnerschaften in Deutschland, 1. Halbjahr 2012, online verfügbar unter: http://www.partnerschaften-deutschland.de/fileadmin/Daten/OEPP-Markt/120827_OEPP_in_Deutschland_Halbjahresbericht_2012.pdf.

ÖPP Deutschland AG (2013): Öffentlich-Private Partnerschaften in Deutschland, 2012.

ÖPP-Kompetenzzentrum Sachsen (2013): Einschätzung der Notwendigkeit und Zulässigkeit der Nutzung von Realwerten für die Wirtschaftlichkeitsbewertung von ÖPP-Projekten. (im Erscheinen).

Pfnür, Andreas; Weiland, Sonja (2009): Empirische Untersuchung der Nutzenwirkungen von PPP Projekten auf den Schulbetrieb am Beispiel der Schulen im Kreis Offenbach, In: Pfnür, Andreas (Hrsg.), Arbeitspapiere zur immobilienwirtschaftlichen Forschung und Praxis, Band Nr. 16, Darmstadt.

Präsidentinnen und Präsidenten der Rechnungshöfe des Bundes und der Länder (201 1): Gemeinsamer Erfahrungsbericht zur Wirtschaftlichkeit von ÖPP-Projekten,

Rademaker, Maike (2006): Dossier: Landkreise drohen mit Privatisierung, in: Financial Times Deutschland, 07.02.2006, online verfügbar unter: http://www.ftd.de/politik/deutschland/:dossier-landkreise-drohen-mit-privatisierung /45113.html. 
Rechnungshof Baden-Württemberg (2009): Wirtschaftlichkeitsanalyse von ÖPP-Projekten der ersten und zweiten Generation bei Hochbaumaßnahmen des Landes, Beratende Äußerungen nach § 88 Abs.2 Landeshaushaltsordnung, online verfügbar unter: http://archiv.baden-wuerttemberg.de/sixcms/media.php/978/PAP0403B\%c4BAU.pdf. Rechnungshof des Freistaates Sachsen (2009): Jahresbericht 2009, online verfügbar unter: http://www.rechnungshof.sachsen.de/jb2009/JB2009.pdf.

Rechnungshof des Freistaates Sachsen (2011): Jahresbericht 2011, Band II: Kommunalfinanzen, Ergebnisse der überörtlichen Kommunalprüfung, online verfügbar unter: http://www.rechnungshof.sachsen.de/jb2011/JB2011Band_Il.pdf.

Reichard, Christoph (2012): Neve institutionelle Varianten öffentlicher Leistungserbringung. In: Schröter, Eckhard/ von Maravic, Patrick/Röber, Jörg (Hrsg.): Zukunftsfähige Verwaltung? Herausforderungen und Lösungsstrategien in Deutschland, Österreich und der Schweiz, S. 207-234.

Reichard, Christoph (2006): Organisations-PPP-Typologie und praktische Ausprägungn, in: Budäus, Dietrich (Hrsg.), Kooperationsformen zwischen Staat und Markt, Baden-Baden, S. 77-94.

Reidenbach, Michael et. al. (2008): Investitionsrückstand und Investitionsbedarf der Kommunen, Ausmaß, Ursachen, Folgen, Strategien, Edition Difu, Band 4/2008, Berlin.

Röber, Manfred (Hrsg.) (2012): Institutionelle Vielfalt und neue Unübersichtlichkeit. Zukunftsperspektiven effizienter Steuerung öffentlicher Aufgaben zwischen Public Management und Public Governance. Schriften zur öffentlichen Verwaltung und öffentlichen Wirtschaft, Band 212, Berlin.

Rösmann, Tobias (2012): Partnerschaften in der Krise, in: Frankfurter Allgemeine Zeitung, 09.08.2012, online verfügbar unter: http://www.faz.net/aktuell/rhein-main/oeffentlich-private-projekte-partnerschaften-in-der-krise-1 1850503.html.

Sack, Detlef (2013): Krise und Organisationswandel von lokaler Governance - Das Beispiel Public Private Partnerships, in: Haus, Michale/Kuhlmann, Sabine (Hrsg.), Lokale Politik und Verwaltung im Zeichen der Krise?, S. 139157.

Simantke, Elisa (2013): Frankfurts Blamage, in: Der Tagesspiegel. online verfügbar unter: http://www.tagesspiegel. de/politik/public-private-partnership-das-gesamte-projekt-scheitert/7621730-3.html.

Tempel, Michael (2010): Macht Stadt bei PPP Minus? In: Mitteldeutsche Zeitung. online verfügbar unter: http:// www.mz-web.de/halle-saalekreis/halle-macht-stadt-bei-ppp-minus-,20640778, 17743394.html.

Wohltmann, Matthias (2012): Kreisfinanzen 2011/2012 - Großer Investitionsstau in den Kreishaushalten, in: Der Landkreis, 82. Jg., Juli/August 2012, S. 337-374. 


\section{Gesetzestexte und Entscheidungen}

GemHKVO (NI): Verordnung über die Aufstellung und Ausführung des Haushaltsplans sowie die Abwicklung der Kassengeschäfte der Gemeinden auf der Grundlage der kommunalen Doppik (Gemeindehaushalts- und -kassenverordnung - GemHKVO -) in der Fassung vom 22. Dezember 2005 (Nds. GVBI. S. 458), zuletzł geändert am 1. Februar 2011 (Nds. GVBl. S. 31).

GemHVO (BW): Verordnung des Innenministeriums über die Haushaltswirtschaft der Gemeinden (Gemeindehaushaltsverordnung - GemHVO) in der Fassung vom 11. Dezember 2009 (GBI. BW S. 770).

GemHVO (HE): Verordnung über die Aufstellung und Ausführung des Haushaltsplans der Gemeinden (Gemeindehaushaltsverordnung - GemHVO -) in der Fassung vom 2. April 2006 (GVBI. I HE S. 235), zuletzł geändert am 27. Dezember 2011 (GVBI. I HE S. 840).

GemHVO (NRW): Verordnung über das Haushaltswesen der Gemeinden im Land Nordrhein-Westfalen (Gemeindehaushaltsverordnung NRW - GemHVO NRW) in der Fassung vom 16. November 2004 (GV.NRW. S. 644), zuletz† geändert am 18. September 2012 (GV.NRW. S. 432).

GemHVO (RP): Gemeindehaushaltsverordnung (GemHVO) in der Fassung vom 18. Mai 2006 (GVBI. RP. S. 203), zuletzt geändert am 6. April 2010 (GVBI. RP. S. 64).

GemHVO-Doppik (MV): Gemeindehaushaltsverordnung-Doppik (GemHVO-Doppik) in der Fassung vom 25. Februar 2008 (GVOBI. M-V S. 34), zuletzł geändert am 13. Dezember 2011 (GVOBI. M-V S. 1118).

GemHVO-Doppik (ST): Verordnung über die Aufstellung und Ausführung des Haushaltsplanes der Gemeinden im Land Sachsen-Anhalt nach den Grundsätzen der doppelten Buchführung (Gemeindehaushaltsverordnung Doppik GemHVO Doppik) in der Fassung vom 22. Dezember 2010 (GVBI. LSA S. 648).

GemHVO-Doppik (SH): Landesverordnung über die Aufstellung und Ausführung eines doppischen Haushaltsplanes der Gemeinden (Gemeindehaushaltsverordnung-Doppik - GemHVO-Doppik) in der Fassung vom 30. August 2012 (GVOBI. Schl.-H. S. 646).

KomHKV (BB): Verordnung über die Aufstellung und Ausführung des Haushaltsplans der Gemeinden (Kommunale Haushalts- und Kassenverordnung- KomHKV) in der Fassung vom 14. Februar 2008 (GVBI.II/08 [03] BB S. 14), zuletzt geändert am 28. Juni 2010 (GVBI.II/10 [38] BB S. 1).

KommHV-Doppik (BY): Verordnung über das Haushalts-, Kassen- und Rechnungswesen der Gemeinden, der Landkreise und der Bezirke nach den Grundsätzen der doppelten kommunalen Buchführung (Kommunalhaushaltsverordnung - Doppik - KommHV-Doppik) in der Fassung vom 5. Oktober 2007 (GVBI. BY S. 678), zuletzt geändert am 3. Januar 2011 (GVBI. BY S. 25).

KommHV-Kameralistik (BY): Verordnung über das Haushalts-, Kassen- und Rechnungswesen der Gemeinden, der Landkreise und der Bezirke nach den Grundsätzen der Kameralistik (Kommunalhaushaltsverordnung - Kameralistik - KommHV-Kameralistik) in der Fassung vom 5. Oktober 2007 (GVBI. BY S. 707), zuletzł geändert am 3. Januar 2011 (GVBI. BY S. 25).

KommHVO (SL): Kommunalhaushaltsverordnung (KommHVO) in der Fassung vom 10. Oktober 2006 (Amtsbl. S. 1842), zuletzt geändert am 5. Oktober 2009 (Amtsbl. S. 1694).

LHO (BE): Landeshaushaltsordnung (LHO) in der Fassung vom 30. Januar 2009 (GVBI. BE S. 31 , ber. S. 486), zuletzł geändert am 5. November 2012 (GVBI. Be S. 354).

LHO (BR): Landeshaushaltsordnung (LHO) in der Fassung vom 25. Mai 1971 (Brem.GBI S. 143), zuletzt geändert am 17. Mai 2011 (Brem.GBI. S. 371).

$\mathrm{LHO}(\mathrm{HH})$ : Haushaltsordnung der Freien und Hansestadt Hamburg (Landeshaushaltsordnung - $\mathrm{LHO}$ ) in der Fassung vom 23. Dezember 1971 (HmbGVBI. 1972, S. 10), zuletzł geändert am 18. Dezember 2012 (HmbGVBI. S. 530). 
SächsKomHVO-Doppik (SN): Verordnung des Sächsischen Staatsministeriums des Innern über die kommunale Haushaltswirtschaft nach den Regeln der Doppik (Sächsische Kommunalhaushaltsverordnung - Doppik - SächsKomHVO-Doppik) in der Fassung vom 8. Februar 2008 (SächsGVBI. S. 202), zuletzł geändert am 19. Dezember 2012 (SächsGVBI. S. 764).

ThürGemHV (TH): Thüringer Verordnung über das Haushalts-, Kassen- und Rechnungswesen der Gemeinden (Thüringer Gemeindehaushaltsverordnung - ThürGemHV -) in der Fassung vom 26. Januar 1993 (GVBI. TH S. 181), zuletzt geändert am 15. September 2006 (GVBI. TH S. 520).

ThürGemHV-Doppik (TH): Thüringer Gemeindehaushaltsverordnung-Doppik (ThürGemHV-Doppik) in der Fassung vom 11. Dezember 2008(GVBI. TH S. 504). 


\section{Impressum}

\section{ÖPP-Kompetenzzentrum Sachsen}

\author{
E: info@öpp-sachsen.de \\ I: www.öpp-sachsen.de
}

\section{Kompetenzzentrum \\ Öffentliche Wirtschaft, Infrastruktur und Daseinsvorsorge e.V.}

E: kompetenzzentrum@wifa.uni-leipzig.de

I: www.wifa.uni-leipzig.de/kompetenzzentrum

Direktoren:

Prof. Dr. Thomas Lenk

Prof. Dr. Manfred Röber

Geschäftsführer:

Dr. Oliver Rottmann

Alle Angaben wurden sorgfältig recherchiert und zusammengestellt. Für die Richtigkeit und Vollständigkeit des Inhalts sowie für zwischenzeitliche Änderungen übernehmen die Herausgeber keine Gewähr. Alle Rechte vorbehalten, auch die der fotomechanischen Wiedergabe und Speicherung in elektronischen Medien.

Leipzig, August 2013

(C) Kompetenzzentrum Öffentliche Wirtschaft, Infrastruktur und Daseinsvorsorge e.V., ÖPP-Kompetenzzentrum Sachsen, Leipzig, August 2013 
Kompetenzzentrum Öffentliche Wirtschaft, Infrastruktur und Daseinsvorsorge e.V.

Neues Augusteum

Augustusplatz 10

04109 Leipzig
ÖPP-Kompetenzzentrum Sachsen

Städtisches Kaufhaus

Universitätstraße 16

04109 Leipzig

Leipzig, August 2013 
Das Kompetenzzentrum für kommunale Infrastruktur Sachsen (KOMKIS) stellt eine kostenfreie Informations- und Beratungsplattform für die kommunale Ebene in Sachsen dar. Im Schnittstellenbereich zwischen Verwaltung, Wissenschaft und Politik agiert das KOMKIS als kompetenter Informationsgeber, neutralen Vermittler und inhaltlicher Ansprechpartner zu Themen der kommunalen Infrastrukturbeschaffung, -erhaltung und -bewirtschaftung.

Weitere Veröffentlichungsformate abrufbar unter www.uni-leipzig.de/komkis:

\section{KOMKIS Dialog}

\section{KOMKIS Position}

\section{KONKIS Praxis}

KOMKIS Report

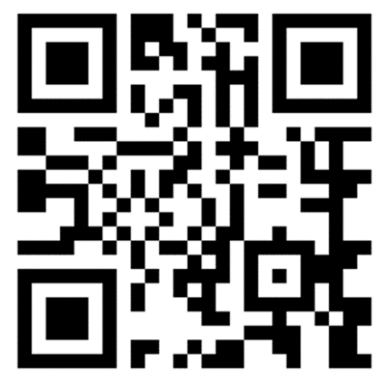

